\title{
Nonleptonic two-body $B$-decays including axial-vector mesons in the final state
}

\author{
G. Calderón* \\ Facultad de Ingeniería Mecánica y Eléctrica, Universidad Autónoma de Coahuila, C.P. 27000, Torreón, Coahuila, México \\ J. H. Muñot讯 and C. E. Verd团 \\ Departamento de Física, Universidad del Tolima A. A. 546, Ibagué, Colombia
}

\begin{abstract}
We present a systematic study of exclusive charmless nonleptonic two-body $B$ decays including axial-vector mesons in the final state. We calculate branching ratios of $B \rightarrow P A, V A$ and $A A$ decays, where $A, V$ and $P$ denote an axial-vector, a vector and a pseudoscalar meson, respectively. We assume naive factorization hypothesis and use the improved version of the nonrelativistic ISGW quark model for form factors in $B \rightarrow A$ transitions. We include contributions that arise from the effective $\Delta B=1$ weak Hamiltonian $H_{\text {eff }}$. The respective factorized amplitude of these decays are explicitly showed and their penguin contributions are classified. We find that decays $B^{-} \rightarrow a_{1}^{0} \pi^{-}$, $\bar{B}^{0} \rightarrow a_{1}^{ \pm} \pi^{\mp}, B^{-} \rightarrow a_{1}^{-} \bar{K}^{0}, \bar{B}^{0} \rightarrow a_{1}^{+} K^{-}, \bar{B}^{0} \rightarrow f_{1} \bar{K}^{0}, B^{-} \rightarrow f_{1} K^{-}, B^{-} \rightarrow K_{1}^{-}(1400) \eta^{\left({ }^{\prime}\right)}$, $B^{-} \rightarrow b_{1}^{-} \bar{K}^{0}$, and $\bar{B}^{0} \rightarrow b_{1}^{+} \pi^{-}\left(K^{-}\right)$have branching ratios of the order of $10^{-5}$. We also study the dependence of branching ratios for $B \rightarrow K_{1} P(V, A)$ decays $\left(K_{1}=K_{1}(1270), K_{1}(1400)\right)$ with respect to the mixing angle between $K_{A}$ and $K_{B}$.
\end{abstract}

PACS numbers: $13.25 . \mathrm{Hw}, 12.38 . \mathrm{Bx}$

\section{INTRODUCTION}

Recent experimental results for $B \rightarrow a_{1} \pi$ and $B \rightarrow K_{1}(1270) \gamma$ decays obtained by BABAR, Belle and CLEO [1] have opened an interesting area of research about production of axial-vector mesons in $B$ decays. Two-body $B$ decays have been considered one of the premier places to understand the interplay of QCD and electroweak interactions, to look for CP violation and over constrain the CKM parameters in the Standard Model. And indeed, exclusive modes $B \rightarrow P P, P V$ and $V V$, which have been extensively discussed in the literature have committed such expectations.

In the search of different alternative modes to the traditional studied, we consider processes which include an axialvector meson in the final state. It is expected that some of these decay channels have large branching ratios [2] and can be within the reach of future experiments. Moreover, they are an additional scenario for understanding QCD and electroweak penguin effects in the Standard Model. These modes give additional and complementary information about exclusive nonleptonic weak decays of $B$ mesons.

The two most important penguin contributions correspond to $a_{4}$ and $a_{6}$ QCD coefficients. These coefficients have different sign in the amplitude $\mathcal{M}(B \rightarrow V P)$, making their contribution small. For $B \rightarrow A P$ decays they have equal sign, thus we have a bigger contribution in penguin sector. Branching ratios of these decays are good candidates to be measured.

Our purpose is to present a systematic analysis about charmless modes $B \rightarrow A P, B \rightarrow A V$ and $B \rightarrow A A$, similar in completeness to previous studies about channels $B \rightarrow P P, B \rightarrow P V$ and $B \rightarrow V V$ which have been extensively considered in the literature $[3,4]$.

There are two types of axial-vector mesons [5]. In spectroscopic notation ${ }^{2 s+1} L_{J}$, these $p$-wave mesons are ${ }^{3} P_{1}$ and ${ }^{1} P_{1}$, with $J^{P C}=1^{++}$and $1^{+-}$, respectively. Under $S U(3)$ flavor symmetry, the ${ }^{3} P_{1}$-nonet is composed by $a_{1}(1260)$, $f_{1}(1285), f_{1}(1420)$, and $K_{1 A}$ and the ${ }^{1} P_{1}$-nonet is integrated by $b_{1}(1235), h_{1}(1170), h_{1}(1380)$, and $K_{1 B}$. However, physical strange axial-vector mesons $K_{1}(1270)$ and $K_{1}(1400)$ are a mixture of $K_{1 A}$ and $K_{1 B}$

$$
\begin{aligned}
& K_{1}(1270)=K_{1 A} \sin \theta+K_{1 B} \cos \theta \\
& K_{1}(1400)=K_{1 A} \cos \theta-K_{1 B} \sin \theta
\end{aligned}
$$

where $\theta$ is the mixing angle.

\footnotetext{
*Electronic address: gecalde@gmail.com

${ }^{\dagger}$ Electronic address: jhmunoz@ut.edu.co

${ }^{\ddagger}$ Electronic address: cvera@ut.edu.co
} 
At theoretical level some authors have worked with production of axial-vector mesons in nonleptonic $B$ decays. Katoch-Verma [6] studied $B \rightarrow P A$ decays at tree level using the factorization hypothesis and the non-relativistic Isgur-Scora-Grinstein-Wise (ISGW) quark model [7]. Nardulli-Pham in Ref. 2] did an analysis of two-body $B$ decays with an axial-vector meson in final state using factorization and the $B \rightarrow K_{1}$ form factors obtained from measured radiative decays. They calculated the branching ratio for $B \rightarrow J / \psi K_{1}$ and derived some predictions for a few nonleptonic decays channels involving light strange or non-strange axial-vector mesons in final state using naive factorization and relations from Heavy Quark Effective Theory. Recently, Laporta-Nardulli-Pham [8] presented an analysis about some charmless $B \rightarrow P A$ decays including contributions of the effective weak Hamiltonian $H_{\text {eff }}$, assuming factorization approach and employing as inputs a limited number of experimental data. They did not use predictions from theoretical models for form factors. In Ref. [9], the authors investigated $B \rightarrow K_{1} \phi$ decays employing the generalized factorization hypothesis and light-front approach for form factors.

Cheng in Ref. [10] studied Cabibbo-allowed hadronic $B$ decays at tree level containing an even-parity charmed meson in final state. In this work, the author predicted branching ratios for some decays of type $B \rightarrow A P(V)$ where $A$ is, in this case, a charmed axial-vector meson. Calculation was performed within the framework of generalized factorization. Form factors for $B \rightarrow A$ transition were calculated with the improved version of the Isgur-Scora-Grinstein-Wise quark model, called ISGW2 [11]. For $B \rightarrow P$ and $B \rightarrow V$ form factors, the author used the Melikhov-Stech model [12]. Recently, Cheng-Chua [13] continue studying even-parity charmed meson production in $B$ decays, calculating $B \rightarrow D^{* *}\left(D^{* *}\right.$ denotes a $p$-wave charmed meson) form factors within the covariant light-front quark model.

Others authors have been interested in radiative $B \rightarrow K_{1}$ decays (see for example Ref. [2]). Recently, J-P. Lee [14] revisited the $B \rightarrow K_{1}$ form factors in the light-cone sum rules, and reduced the discrepancy between theoretical prediction and experimental data reported by Belle Collaboration [1] for $B \rightarrow K_{1} \gamma$. Lee claims that it is necessary more information about the mixing angle between $K_{1 A}$ and $K_{1 B}$ to reduce theoretical uncertainties. In fact, this mixing angle has been estimated by some different methods [15]. However there is not yet a consensus about its value [16].

$\mathrm{CP}$ violation effects have been also investigated in nonleptonic $B$ decays with axial-vector mesons in the final state. For example, in Ref. [17] time-dependent CP asymmetries in $\bar{B}^{0} \rightarrow D^{*-} a_{1}^{+}$are studied in order to learn about the linear combination of weak phases $(2 \beta+\gamma)$. And more recently (see Ref. [18]), in an analysis of $B^{0} \rightarrow a_{1}^{ \pm} \pi^{\mp}$ modes, they determined the phase $\alpha_{e f f}$, which include the weak phase $\alpha$ and effects due to penguin contribution. Moreover, applying $S U(3)$ symmetry to these decays and to $B \rightarrow a_{1} K$ and $B \rightarrow K_{1} \pi$, they obtained bounds on $\left(\alpha-\alpha_{e f f}\right)$.

In this paper we are interested in studying exclusive charmless nonleptonic two-body $B$ decays including axialvector mesons in the final state. We present an overview and a systematic study about this type of processes. For this, we compute branching ratios for exclusive channels $B \rightarrow A P, A V, A A$ that are allowed by the CKM factors, including contributions of the effective weak Hamiltonian $H_{\text {eff }}$ (tree and penguin), assuming the naive factorization hypothesis and using the improved version of the ISGW [11] quark model for calculating the respective form factors related with $B \rightarrow A$ transitions. Form factors for $B \rightarrow P$ and $B \rightarrow V$ transitions have been taken from the relativistic Wirbel-Stech-Bauer (WSB) quark model [19] and from Light Cone Sum Rules (LCSR) [20].

This paper is organized as follows: In Sec. II we discuss the effective weak Hamiltonian, effective Wilson coefficients and naive factorization hypothesis. Input parameters and mixing schemes are discussed in Sec. III. In Sec. IV we present form factors for $B \rightarrow P(V)$ transitions taken from the WSB model and LCSR approach, and $B \rightarrow A$ transitions calculated in ISGW2 model. In Sec. V is discussed the amplitudes and manner to calculate branching ratios for processes considered. Numerical results for branching ratios are presented in Sec. VI. We conclude in Sec. VII with a summary. Amplitudes for all charmless $B \rightarrow A P, A V$ and $A A$ processes are given explicitly in the appendices.

\section{EFFECTIVE HAMILTONIAN AND FACTORIZATION}

The basis for the study of two-body charmless hadronic $B$-decays is the effective weak Hamiltonian $H_{\text {eff }}$ [21]. For $\Delta B=1$ transitions it can be written as

$$
\begin{aligned}
H_{e f f}= & \frac{G_{F}}{\sqrt{2}}\left[V_{u b} V_{u q}^{*}\left(C_{1}(\mu) O_{1}^{u}(\mu)+C_{2}(\mu) O_{2}^{u}(\mu)\right)+V_{c b} V_{c q}^{*}\left(C_{1}(\mu) O_{1}^{c}(\mu)+C_{2}(\mu) O_{2}^{c}(\mu)\right)\right. \\
& \left.-V_{t b} V_{t q}^{*}\left(\sum_{i=3}^{10} C_{i}(\mu) O_{i}(\mu)+C_{g}(\mu) O_{g}(\mu)\right)\right]+ \text { h.c. },
\end{aligned}
$$

where $G_{F}$ is the Fermi constant and $C_{i}(\mu)$ are the Wilson coefficients evaluated at the renormalization scale $\mu$. Local operators $O_{i}(\mu)$ are given below for $b \rightarrow q$ transitions 


$$
\begin{aligned}
O_{1}^{u} & =\bar{q}_{\alpha} \gamma^{\mu} L u_{\alpha} \cdot \bar{u}_{\beta} \gamma_{\mu} L b_{\beta} \\
O_{2}^{u} & =\bar{q}_{\alpha} \gamma^{\mu} L u_{\beta} \cdot \bar{u}_{\beta} \gamma_{\mu} L b_{\alpha} \\
O_{1}^{c} & =\bar{q}_{\alpha} \gamma^{\mu} L c_{\alpha} \cdot \bar{c}_{\beta} \gamma_{\mu} L b_{\beta} \\
O_{2}^{c} & =\bar{q}_{\alpha} \gamma^{\mu} L c_{\beta} \cdot \bar{c}_{\beta} \gamma_{\mu} L b_{\alpha} \\
O_{3(5)} & =\bar{q}_{\alpha} \gamma^{\mu} L b_{\alpha} \cdot \sum_{q^{\prime}} \bar{q}_{\beta}^{\prime} \gamma_{\mu} L(R) q_{\beta}^{\prime} \\
O_{4(6)} & =\bar{q}_{\alpha} \gamma^{\mu} L b_{\beta} \cdot \sum_{q^{\prime}} \bar{q}_{\beta}^{\prime} \gamma_{\mu} L(R) q_{\alpha}^{\prime} \\
O_{7(9)} & =\frac{3}{2} \bar{q}_{\alpha} \gamma^{\mu} L b_{\alpha} \cdot \sum_{q^{\prime}} e_{q^{\prime}} \bar{q}_{\beta}^{\prime} \gamma_{\mu} R(L) q_{\beta}^{\prime} \\
O_{8(10)} & =\frac{3}{2} \bar{q}_{\alpha} \gamma^{\mu} L b_{\beta} \cdot \sum_{q^{\prime}} e_{q^{\prime}} \bar{q}_{\beta}^{\prime} \gamma_{\mu} R(L) q_{\alpha}^{\prime} \\
O_{g} & =\left(g_{s} / 8 \pi^{2}\right) m_{b} \bar{q}_{\alpha} \sigma^{\mu \nu} R\left(\lambda_{\alpha \beta}^{A} / 2\right) b_{\beta} G_{\mu \nu}^{A},
\end{aligned}
$$

where $q$ can be the quarks $d$ or $s$. $L$ and $R$ stand for left and right projectors defined as $\left(1-\gamma_{5}\right)$ and $\left(1+\gamma_{5}\right)$, respectively. The symbols $\alpha$ and $\beta$ are $S U(3)$ color indices and $\lambda_{\alpha \beta}^{A}(A=1, \ldots, 8)$ are the Gell-Mann matrices. The sums run over active quarks at the scale $\mu=\mathcal{O}\left(m_{b}\right)$, i.e. $q^{\prime}$ runs with the quarks $u, d, s$ and $c$.

We use the next to leading order Wilson coefficients for $\Delta B=1$ transitions obtained in the naive dimensional regularization scheme (NDR) at the energy scale $\mu=m_{b}\left(m_{b}\right), \Lambda \frac{(5)}{M S}=225 \mathrm{MeV}$ and $m_{t}=170 \mathrm{GeV}$. These values are $c_{1}=1.082, c_{2}=-0.185, c_{3}=0.014, c_{4}=-0.035, c_{5}=0.009, c_{6}=-0.041, c_{7} / \alpha=-0.002, c_{8} / \alpha=0.054$, $c_{9} / \alpha=-1.292$ and $c_{10} / \alpha=0.263$, where $\alpha$ is the fine structure constant, see Table XXII in Ref. [21].

In order to calculate the amplitude for a nonleptonic two-body $B \rightarrow M_{1} M_{2}$ decay, we use the effective weak Hamiltonian $H_{e f f}$,

$$
\mathcal{M}\left(B \rightarrow M_{1} M_{2}\right)=\left\langle M_{1} M_{2}\left|H_{e f f}\right| B\right\rangle=\frac{G_{F}}{\sqrt{2}} \sum_{i} C_{i}(\mu)\left\langle M_{1} M_{2}\left|O_{i}(\mu)\right| B\right\rangle
$$

Hadronic matrix elements $\left\langle O_{i}(\mu)\right\rangle \equiv\left\langle M_{1} M_{2}\left|O_{i}(\mu)\right| B\right\rangle$ can be evaluated under factorization hypothesis, which approximates hadronic matrix element by product of two matrix elements of singlet currents. These currents are parametrized by decay constants and form factors. It is necessary to point out that these matrix elements as products of conserved currents are $\Lambda_{\overline{M S}}, \mu$ scale and renormalization scheme independent [22, 23]. The suggested energy scale to apply factorization for $B$ decays is $\mu_{f}=\mathcal{O}\left(m_{b}\right)$. Besides this simple approximation, it is well established that nonfactorisable contributions must be present in the matrix elements in order to cancel the scale $\mu$ and renormalization scheme dependence of $C_{i}(\mu)$.

To solve the issue of scale $\mu$ dependence, but not the renormalization scheme dependence [24], it is proposed in Refs. 3, 4], to isolate from the matrix element $\left\langle O_{i}(\mu)\right\rangle$ the $\mu$ dependence, and link with the $\mu$ dependence in the Wilson coefficients $C_{i}(\mu)$ to form $c_{i}^{\text {eff }}$, effective Wilson coefficients independent of $\mu$. Matrix elements $\left\langle O_{i}\right\rangle_{\text {tree }}$ and effective $c_{i}^{e f f}$ Wilson coefficients are scale $\mu$ independent, so the amplitude. Thus, we can write

$$
\sum_{i} C_{i}(\mu)\left\langle O_{i}(\mu)\right\rangle=\sum_{i} C_{i}(\mu) g_{i}(\mu)\left\langle O_{i}\right\rangle_{\text {tree }}=\sum_{i} c_{i}^{e f f}\left\langle O_{i}\right\rangle_{\text {tree }}
$$

The formula for effective Wilson coefficients and their numerical values have been given explicitly in Ref. [4]. These values depend on quark masses, CKM parameters and renormalization scheme. In this article we recalculate the effective Wilson coefficient $c_{i}^{\text {eff }}$, because there have been some changes in the CKM parameters since the authors of Ref. [4] calculated them. We choose naive dimensional regularization scheme to calculate. We present the effective Wilson coefficients $c_{i}^{\text {eff }}$ in Table I, for $b \rightarrow d$ and $b \rightarrow s$ transitions evaluated at the factorization scale $\mu_{f}=m_{b}$, averaged momentum transfer $k^{2}=m_{b}^{2} / 2$ and current CKM parameters, see Sec. III.

The effective Wilson coefficients appear in decay amplitudes as linear combinations. This allows to define $a_{i}$ coefficients, which encode dynamics of the decay, by 


$$
\begin{aligned}
& a_{i} \equiv c_{i}^{\text {eff }}+\frac{1}{N_{c}} c_{i+1}^{\text {eff }}(i=\text { odd }) \\
& a_{i} \equiv c_{i}^{\text {eff }}+\frac{1}{N_{c}} c_{i-1}^{\text {eff }}(i=\text { even })
\end{aligned}
$$

where index $i=1, \ldots, 10$ and $N_{c}=3$ is the color number. In Table II, we give the $a_{i}$ values for $b \rightarrow d$ and $b \rightarrow s$ transitions calculated with the effective Wilson coefficients $c_{i}^{e f f}$, given in Table I.

TABLE I. Effective Wilson coefficients $c_{i}^{\text {eff }}$ for $b \rightarrow d$ and $b \rightarrow s$ transitions. Evaluated at $\mu_{f}=m_{b}$ and $k^{2}=m_{b}^{2} / 2$, where we use the Wolfenstein parameters $\lambda=0.2272, A=0.818, \rho=0.227$ and $\eta=0.349$, see Sec. III.

\begin{tabular}{lcc}
\hline \hline$c_{i}^{\text {eff }}$ & $b \rightarrow d$ & $b \rightarrow s$ \\
\hline$c_{1}^{\text {eff }}$ & 1.1680 & 1.1680 \\
$c_{2}^{\text {eff }}$ & -0.3652 & -0.3652 \\
$c_{3}^{\text {eff }}$ & $0.0233+\mathrm{i} 0.0036$ & $0.0233+\mathrm{i} 0.0043$ \\
$c_{4}^{\text {eff }}$ & $-0.0481-\mathrm{i} 0.0109$ & $-0.0482-\mathrm{i} 0.0129$ \\
$c_{5}^{\text {eff }}$ & $0.0140+\mathrm{i} 0.0036$ & $0.0140+\mathrm{i} 0.0043$ \\
$c_{6}^{\text {eff }}$ & $-0.0503-\mathrm{i} 0.0109$ & $-0.0504-\mathrm{i} 0.0129$ \\
$c_{7}^{\text {eff }} / \alpha$ & $-0.0310-\mathrm{i} 0.0317$ & $-0.0312-\mathrm{i} 0.0357$ \\
$c_{8}^{\text {eff }} / \alpha$ & 0.0551 & 0.0551 \\
$c_{9}^{\text {eff }} / \alpha$ & $-1.4275-\mathrm{i} 0.0317$ & $-1.4277-\mathrm{i} 0.0357$ \\
$c_{10}^{\text {eff }} / \alpha$ & 0.4804 & 0.4804 \\
\hline \hline
\end{tabular}

TABLE II. Effective coefficients $a_{i}$ for $b \rightarrow d$ and $b \rightarrow s$ transitions (in unit of $10^{-4}$ for $a_{3}, \ldots, a_{10}$ ).

\begin{tabular}{lcc}
\hline \hline$a_{i}$ & $b \rightarrow d$ & $b \rightarrow s$ \\
\hline$a_{1}$ & 1.046 & 1.046 \\
$a_{2}$ & 0.024 & 0.024 \\
$a_{3}$ & 72 & 72 \\
$a_{4}$ & $-403-\mathrm{i} 97$ & $-404-\mathrm{i} 115$ \\
$a_{5}$ & -28 & -28 \\
$a_{6}$ & $-456-\mathrm{i} 97$ & $-457-\mathrm{i} 115$ \\
$a_{7}$ & $-0.92-\mathrm{i} 2.31$ & $-0.94-\mathrm{i} 2.61$ \\
$a_{8}$ & $3.26-\mathrm{i} 0.77$ & $3.26-\mathrm{i} 0.87$ \\
$a_{9}$ & $-92.5-\mathrm{i} 2.31$ & $-92.5-\mathrm{i} 2.61$ \\
$a_{10}$ & $0.33-\mathrm{i} 0.77$ & $0.33-\mathrm{i} 0.87$ \\
\hline \hline
\end{tabular}

\section{INPUT PARAMETERS}

We parametrize the CKM matrix in terms of the Wolfenstein parameters $\lambda, A, \bar{\rho}$ and $\bar{\eta}$ [25]

$$
\left(\begin{array}{ccc}
1-\frac{1}{2} \lambda^{2} & \lambda & A \lambda^{3}(\rho-i \eta) \\
-\lambda & 1-\frac{1}{2} \lambda^{2} & A \lambda^{2} \\
A \lambda^{3}(1-\bar{\rho}-i \bar{\eta}) & -A \lambda^{2} & 1
\end{array}\right)
$$

with $\bar{\rho}=\rho\left(1-\lambda^{2} / 2\right)$ and $\bar{\eta}=\eta\left(1-\lambda^{2} / 2\right)$, including $\mathcal{O}\left(\lambda^{5}\right)$ corrections [26].

By a global fit that uses all available measurements and that imposes unitary constrains, the Wolfenstein parameters are precisely determined. There exist two ways to combining experimental data, the frequentist statistics [27] and the Bayesian approach [28], providing similar results. Thus, we take for the Wolfenstein parameters the central values $\lambda=0.2272, A=0.818, \bar{\rho}=0.221$ and $\bar{\eta}=0.340$ [5].

The running quark masses are necessary in calculation of penguin terms in the amplitude where appear scalar and pseudoscalar matrix elements which are reduced by use of Dirac equation of motion. Running quark masses are given 
at the scale $\mu \approx m_{b}$, since energy released in $B$ decays is of order $m_{b}$. We use $m_{u}\left(m_{b}\right)=3.2 \mathrm{MeV}, m_{d}\left(m_{b}\right)=6.4$ $\mathrm{MeV}, m_{s}\left(m_{b}\right)=127 \mathrm{MeV}, m_{c}\left(m_{b}\right)=0.95 \mathrm{GeV}$ and $m_{b}\left(m_{b}\right)=4.34 \mathrm{GeV}$, see Ref. [29].

Decay constants of pseudoscalar and vector mesons are well determined experimentally. We use the following values [5]: $f_{\pi}=130.7 \mathrm{MeV}, f_{K}=160 \mathrm{MeV}, f_{\rho}=216 \mathrm{MeV}, f_{\omega}=195 \mathrm{MeV}, f_{K^{\star}}=221 \mathrm{MeV}$ and $f_{\phi}=237 \mathrm{MeV}$.

The $\omega-\phi, \rho^{0}-\omega, \eta-\eta^{\prime}$ and $K_{1 A}-K_{1 B}$ mixing are introduced through mixing in decay constants and form factors. We consider ideal mixing for the system $(\omega, \phi)$, i.e. $\omega=1 / \sqrt{2}(u \bar{u}+d \bar{d})$ and $\phi=s \bar{s}$. In next section we will discuss mixing in form factors. In the following we describe mixing in decay constants.

For the $\eta-\eta^{\prime}$ mixing we use the two mixing angle formalism proposed in [30, 31], which define physical states $\eta$ and $\eta^{\prime}$ in terms of flavor octet and singlet, $\eta_{8}$ and $\eta_{0}$, respectively:

$$
\begin{aligned}
& |\eta\rangle=\cos \theta_{8}\left|\eta_{8}\right\rangle-\sin \theta_{0}\left|\eta_{0}\right\rangle, \\
& \left|\eta^{\prime}\right\rangle=\sin \theta_{8}\left|\eta_{8}\right\rangle+\cos \theta_{0}\left|\eta_{0}\right\rangle .
\end{aligned}
$$

We introduce decay constants for $\eta_{8}$ and $\eta_{0}$ by $\left\langle 0\left|A_{\mu}^{8}\right| \eta^{\left({ }^{\prime}\right)}(p)\right\rangle=i f_{\eta^{\left({ }^{\prime}\right)}}^{8} p_{\mu}$ and $\left\langle 0\left|A_{\mu}^{0}\right| \eta^{\left({ }^{\prime}\right)}(p)\right\rangle=i f_{\eta^{\left({ }^{\prime}\right)}}^{0} p_{\mu}$. Considering that $\eta_{8}$ and $\eta_{0}$ in terms of quarks are

$$
\begin{aligned}
& \left|\eta_{8}\right\rangle=\frac{1}{\sqrt{6}}|\bar{u} u+\bar{d} d-2 \bar{s} s\rangle, \\
& \left|\eta_{0}\right\rangle=\frac{1}{\sqrt{3}}|\bar{u} u+\bar{d} d+\bar{s} s\rangle,
\end{aligned}
$$

induce a two-mixing angle in decay constants $f_{\eta^{\left({ }^{\prime}\right)}}^{q}$, defined by $\left\langle 0\left|\bar{q} \gamma_{\mu} \gamma_{5} q\right| \eta^{\left({ }^{\prime}\right)}(p)\right\rangle=i f_{\eta^{\left({ }^{\prime}\right)}}^{q} p_{\mu}$,

$$
\begin{aligned}
& f_{\eta^{\prime}}^{u}=\frac{f_{8}}{\sqrt{6}} \sin \theta_{8}+\frac{f_{0}}{\sqrt{3}} \cos \theta_{0} \\
& f_{\eta^{\prime}}^{s}=-2 \frac{f_{8}}{\sqrt{6}} \sin \theta_{8}+\frac{f_{0}}{\sqrt{3}} \cos \theta_{0}
\end{aligned}
$$

and

$$
\begin{aligned}
& f_{\eta}^{u}=\frac{f_{8}}{\sqrt{6}} \cos \theta_{8}-\frac{f_{0}}{\sqrt{3}} \sin \theta_{0} \\
& f_{\eta}^{s}=-2 \frac{f_{8}}{\sqrt{6}} \cos \theta_{8}-\frac{f_{0}}{\sqrt{3}} \cos \theta_{0} .
\end{aligned}
$$

From a complete phenomenological fit of the $\eta-\eta^{\prime}$ mixing parameters in Ref. [31] we have $\theta_{8}=-21.1^{\circ}, \theta_{0}=-9.2^{\circ}$, $\theta=-15.4^{\circ}, f_{8}=165 \mathrm{MeV}$ and $f_{0}=153 \mathrm{MeV}$. Replacing values in Eqs. (10]11), the decay constants are $f_{\eta^{\prime}}^{u}=61.8$ $\mathrm{MeV}, f_{\eta^{\prime}}^{s}=138 \mathrm{MeV}, f_{\eta}^{u}=76.2 \mathrm{MeV}$ and $f_{\eta}^{s}=-110.5 \mathrm{MeV}$. To include in the mixing scheme the $\eta_{c}$, in the calculation we use decay constants defined by $\left\langle 0\left|\bar{c} \gamma_{\mu} \gamma_{5} c\right| \eta^{\left({ }^{\prime}\right)}\right\rangle=i f_{\eta^{\left({ }^{\prime}\right)}}^{q} p_{\mu}$ as are obtained in Ref. [31]: $f_{\eta}^{c}=-(2.4 \pm 0.2)$ $\mathrm{MeV}$ and $f_{\eta^{\prime}}^{c}=-(6.3 \pm 0.6) \mathrm{MeV}$.

In evaluating hadron matrix elements of scalar and pseudoscalar densities in some penguin terms the anomaly must be included in order to ensure a correct chiral behavior for that matrix elements. The expressions are [32]

$$
\begin{aligned}
& \left\langle\eta^{\left({ }^{\prime}\right)}\left|\bar{u} \gamma_{5} u\right| 0\right\rangle=\left\langle\eta^{\left({ }^{\prime}\right)}\left|\bar{d} \gamma_{5} d\right| 0\right\rangle=r_{\eta^{\left({ }^{\prime}\right)}}\left\langle\eta^{\left({ }^{\prime}\right)}\left|\bar{s} \gamma_{5} s\right| 0\right\rangle, \\
& \left\langle\eta^{\left({ }^{\prime}\right)}\left|\bar{s} \gamma_{5} s\right| 0\right\rangle=-i \frac{m_{\eta^{\left({ }^{\prime}\right)}}^{2}}{2 m_{s}}\left(f_{\eta^{\left({ }^{\prime}\right)}}^{s}-f_{\eta^{\left({ }^{\prime}\right)}}^{u}\right),
\end{aligned}
$$

where the ratios $r_{\eta^{\prime}}$ and $r_{\eta}$ are defined by

$$
\begin{aligned}
& r_{\eta^{\prime}}=\frac{\sqrt{2 f_{0}^{2}-f_{8}}}{\sqrt{2 f_{8}^{2}-f_{0}^{2}}} \frac{\cos \theta+(1 / \sqrt{2}) \sin \theta}{\cos \theta-\sqrt{2} \sin \theta}, \\
& r_{\eta}=-\frac{1}{2} \frac{\sqrt{2 f_{0}^{2}-f_{8}}}{\sqrt{2 f_{8}^{2}-f_{0}^{2}}} \frac{\cos \theta-\sqrt{2} \sin \theta}{\cos \theta+(1 / \sqrt{2}) \sin \theta},
\end{aligned}
$$


the numerical values obtained are $r_{\eta^{\prime}}=0.462$ and $r_{\eta}=-0.689$.

The physical states $K_{1}(1270)$ and $K_{1}(1400)$ result from the mixing of $K_{1 A}$ and $K_{1 B},{ }^{3} P_{1}$ and ${ }^{1} P_{1}$ mesons, respectively, see Eq. (1). From experimental data on masses and partial ratios of $K_{1}(1270)$ and $K_{1}(1400)$, it is found two solutions for the mixing angle with a two-fold ambiguity, $\theta= \pm 32^{\circ}$ and $\theta= \pm 58^{\circ}$. The masses for the states $K_{1 A}$ and $K_{1 B}$ are $m_{K_{1 A}}=1367 \mathrm{MeV}$ and $m_{K_{1 B}}=1310 \mathrm{MeV}$, respectively. From $\tau$ decays, the decay constants of the physical states are determined. The values obtained are $f_{K 1}(1270)=171 \mathrm{MeV}$ and $f_{K 1}(1400)=126 \mathrm{MeV}$ [2], using data from Ref. [5].

Thus, we have experimental information to determine decay constants for strange axial-vector mesons. That is no the case for non-strange axial-vector mesons. But, using the mixing angle of the system $K_{A}-K_{B}$ and $S U(3)$ symmetry it is derived decay constants for axial-vector mesons: $\left(f_{b_{1}}, f_{a_{1}}\right)=(74,215) \mathrm{MeV}$ for $\theta=32^{\circ}$ and $\left(f_{b_{1}}, f_{a_{1}}\right)=(-28,223)$ $\mathrm{MeV}$ for $\theta=58^{\circ}$, see Ref. [2]. Since, $f_{1}$ and $h_{1}$ are in the same nonet that $a_{1}$ and $b_{1}$, respectively, by $S U(3)$ symmetry we consider equal decay constants. In the calculations of branching ratios we use the values $f_{a_{1}}=f_{f_{1}}=215 \mathrm{MeV}$ and $f_{b_{1}}=f_{h_{1}}=74 \mathrm{MeV}$. However, in the exact limit of $S U(3)$ symmetry we have $f_{b_{1}}=f_{h_{1}}=0$. We calculate branching ratios and determine the modes which change in that limit.

Matrix elements for $B \rightarrow K_{1}$ transitions are calculated in the flavor base $K_{1 A}-K_{1 B}$. In calculation of amplitude involving a final physical state as $K_{1}(1270)$ or $K_{1}(1400)$ we transform matrix elements from the flavor base to the physical base using Eq. (1).

We use for $B$ meson lifetime $\tau_{B^{-}}=(1.638 \pm 0.011) \times 10^{-12} s$ and $\tau_{B^{0}}=(1.530 \pm 0.009) \times 10^{-12} s$, see Ref. [5], necessary to calculate branching ratios.

\section{FORM FACTORS}

As was stated in Sec. II, hadronic matrix elements $\left\langle O_{i}\right\rangle_{\text {tree }}$ are given in the factorization hypothesis in terms of decay constants and form factors. Unfortunately, due to nonperturbative nature of these matrix elements, there is no complete reliable calculations and only model dependent evaluations are used for them.

We use the WSB model and LCSR approach to determine form factors for $B \rightarrow P$ and $B \rightarrow V$ transitions. In the WSB model and LCSR approach, the form factors for $B \rightarrow A$ transitions have not been calculated. Thus we calculate form factors for $B \rightarrow A$ transitions in the ISGW2 model [11]. In the following subsections we give relevant information to calculate form factors in the respective models.

\section{A. Form factors for $B \rightarrow P(V)$ in the WSB model and LCSR approach}

In the WSB quark model meson-meson matrix elements of currents are evaluated from the overlap integrals of corresponding wave functions, which are solutions of a relativistic harmonic oscillator potential. For momentum transfer squared $q^{2}$ dependence of form factors in the region where $q^{2}$ is not too large, we shall use a single pole dominance ansatz, namely

$$
f\left(q^{2}\right)=\frac{f(0)}{\left(1-q^{2} / m_{*}^{2}\right)},
$$

where $m_{*}$ is the pole mass and $f(0)$ the form factor at zero momentum transfer given in Ref. [19]. Note that the original WSB quark model assumes a monopole behavior for all form factors.

The WSB model has been quite successful in accommodating data in an important number of exclusive semileptonic and nonleptonic two-body decays of $D$ and $B$ mesons.

Form factors for $B \rightarrow P$ transitions are defined as follows

$$
\left\langle P\left(p_{P}\right)\left|V_{\mu}\right| B\left(p_{B}\right)\right\rangle \equiv\left[\left(p_{B}+p_{P}\right)_{\mu}-\frac{m_{B}^{2}-m_{P}^{2}}{q^{2}} q_{\mu}\right] F_{1}\left(q^{2}\right)+\left[\frac{m_{B}^{2}-m_{P}^{2}}{q^{2}}\right] q_{\mu} F_{0}\left(q^{2}\right),
$$

where $q=\left(p_{B}-p_{P}\right)$, as well as form factors for $B \rightarrow V$ transitions by 


$$
\begin{aligned}
\left\langle V\left(p_{V}, \epsilon\right)\left|\left(V_{\mu}-A_{\mu}\right)\right| B\left(p_{B}\right)\right\rangle \equiv & -\epsilon_{\mu \nu \alpha \beta} \epsilon^{\nu *} p_{B}^{\alpha} p_{V}^{\beta} \frac{2 V\left(q^{\prime 2}\right)}{\left(m_{B}+m_{V}\right)}-i\left[\left(\epsilon_{\mu}^{*}-\frac{\epsilon^{*} \cdot q^{\prime}}{q^{\prime 2}} q_{\mu}^{\prime}\right)\left(m_{B}+m_{V}\right) A_{1}\left(q^{2}\right)\right. \\
& \left.-\left(\left(p_{B}+p_{V}\right)_{\mu}-\frac{\left(m_{B}^{2}-m_{V}^{2}\right)}{q^{\prime 2}} q_{\mu}^{\prime}\right)\left(\epsilon^{*} \cdot q^{\prime}\right) \frac{A_{2}\left(q^{\prime 2}\right)}{\left(m_{B}+m_{V}\right)}+\frac{2 m_{V}\left(\epsilon^{*} \cdot q^{\prime}\right)}{q^{\prime 2}} q_{\mu} A_{0}\left(q^{\prime 2}\right)\right]
\end{aligned}
$$

where $q^{\prime}=\left(p_{B}-p_{V}\right)$ and $\epsilon$ is the polarization vector of $V$. In order to cancel the poles at $q^{2}=0$, we must impose restrictions over form factors

$$
\begin{aligned}
F_{1}(0) & =F_{0}(0), \\
2 m_{V} A_{0}(0) & =\left(m_{B}+m_{V}\right) A_{1}(0)-\left(m_{B}-m_{V}\right) A_{2}(0) .
\end{aligned}
$$

In Table III form factors are given for transitions required in calculations: form factors for $B \rightarrow \pi, B \rightarrow K, B \rightarrow \eta$, $B \rightarrow \eta^{\prime}, B \rightarrow \rho, B \rightarrow K^{*}$ and $B \rightarrow \omega$ are evaluated at the $q^{2}=0$ momentum transfer. With respect to $B \rightarrow \eta$ and $B \rightarrow \eta^{\prime}$ transitions the WSB model does not include the $\eta-\eta^{\prime}$ mixing effect. We better consider $S U(3)$ symmetry and use the relations $F_{0}^{B \pi}(0)=\sqrt{3} F^{B \eta_{0}}(0)=\sqrt{6} F^{B \eta_{8}}(0)$, calculating physical form factors from

$$
\begin{aligned}
F^{B \eta} & =F^{B \eta_{8}} \cos \theta-F^{B \eta_{0}} \sin \theta, \\
F^{B \eta^{\prime}} & =F^{B \eta_{8}} \sin \theta+F^{B \eta_{0}} \cos \theta,
\end{aligned}
$$

for $F^{B \pi}(0)=0.333$ and the mixing angle $\theta=-15.4^{\circ}$ [31], we obtain the values $F^{B \eta}(0)=0.181$ and $F^{B \eta^{\prime}}(0)=0.148$. The $\rho^{0}-\omega$ mixing and isospin breaking effects are introduced in hadronic matrix elements $B \rightarrow \rho^{0}$, following Ref. 33]. In the limit of isospin symmetry isospin eigenstates $\rho^{I}$ and $\omega^{I}$ expressed in the flavor basis are $\rho^{I}=(u \bar{u}-d \bar{d}) / \sqrt{2}$ and $\omega^{I}=(u \bar{u}+d \bar{d}) / \sqrt{2}$. The physical states $\rho^{0}$ and $\omega$ are expressed in term of $\rho^{I}$ and $\omega^{I}$ by

$$
\begin{aligned}
& \left|\rho^{0}\right\rangle=\left|\rho^{I}\right\rangle+\epsilon\left|\omega^{I}\right\rangle=\frac{1}{\sqrt{2}}(1+\epsilon)|u \bar{u}\rangle+\frac{1}{\sqrt{2}}(-1+\epsilon)|d \bar{d}\rangle \\
& |\omega\rangle=\left|\omega^{I}\right\rangle-\epsilon^{\prime}\left|\rho^{I}\right\rangle=\frac{1}{\sqrt{2}}\left(1-\epsilon^{\prime}\right)|u \bar{u}\rangle+\frac{1}{\sqrt{2}}\left(1+\epsilon^{\prime}\right)|d \bar{d}\rangle,
\end{aligned}
$$

where the numerical values for mixing parameters are $(1+\epsilon)=(0.092+0.016 i)$ and $\left(1-\epsilon^{\prime}\right)=(1.011+0.030 i)$. The hadronic matrix elements for the $B \rightarrow \rho^{0}$ and $B \rightarrow \omega$ transitions including isospin effects change by the factor $(1+\epsilon)$ and $\left(1-\epsilon^{\prime}\right)$, respectively. The effect in $B \rightarrow \omega$ transitions is negligible and it is not included in branching ratios predictions.

In the LCSR approach form factors for B decays are given in terms of the correlation function of the weak current and the current with quantum numbers of B meson, evaluated between the vacuum and a pseudoscalar or a vector meson. The like cone expansion allows to calculate in the large virtualities of these currents. In the short virtualities regime, the LCSR approach depends on the factorization of correlation function into nonpertubative and universal hadron function amplitudes which are convoluted with process depend amplitudes.

In Ref. [20] form factors for $B \rightarrow P$ and $B \rightarrow V$ transitions are calculated in the LCSR approach. In Table III form factors values at zero momentum transfer are showed, for the set 2 of parameters, taken from Ref. [20]. For the $q^{2}$ dependency of the form factors we use the fit parametrization done in Ref. [20], valid for the full kinematic regime.

\section{B. Form factors for $B \rightarrow A$ in the ISGW2 model}

The ISGW2 model is based in a nonrelativistic constituent quark representation. In the original ISGW model [7] form factors only depend on the maximum momentum transfer, $q^{2}=q_{m}^{2}$. In this model form factor dependence is proportional to $\exp \left[-\left(q_{m}^{2}-q^{2}\right)\right]$, consequently the form factors diminish exponentially as function of $\left(q_{m}^{2}-q^{2}\right)$. This behavior has been improved in the ISGW2 model [1] by expressing the $q^{2}$ dependence as a polynomial term which must be multiplied by a factor which depends on the hyperfine mass. In addition, the improved model incorporates constrains imposed by heavy quark symmetry, hyperfine distortions of wave functions and a more real high recoil behavior. 
TABLE III. Form factors at zero momentum transfer for $B \rightarrow P$ and $B \rightarrow V$ transitions, evaluated in the WSB quark model [19] and LCSR [20].

\begin{tabular}{lccccc}
\hline \hline Transition & $F_{1}=F_{0}$ & $V$ & $A_{1}$ & $A_{2}$ & $A_{3}=A_{0}$ \\
\hline$B \rightarrow \pi$ & $0.333[0.258]$ & & & & \\
$B \rightarrow K$ & $0.379[0.331]$ & & & & \\
$B \rightarrow \eta$ & $0.168[0.275]$ & & & & \\
$B \rightarrow \eta^{\prime}$ & $0.114[-]$ & & & & \\
$B \rightarrow \rho$ & & $0.329[0.323]$ & $0.283[0.242]$ & $0.283[0.221]$ & $0.281[0.303]$ \\
$B \rightarrow \omega$ & & $0.232[0.311]$ & $0.199[0.233]$ & $0.199[0.181]$ & $0.198[0.363]$ \\
$B \rightarrow K^{*}$ & & $0.369[0.293]$ & $0.328[0.219]$ & $0.331[0.198]$ & $0.321[0.281]$ \\
\hline \hline
\end{tabular}

We have made use of the ISGW2 model [1] to determine form factors for $B \rightarrow A$ transitions. The vector and axial part of matrix element for these transitions are parametrized as

$$
\begin{aligned}
\left\langle A\left(p_{A}, \epsilon\right)\left|\left(V_{\mu}-A_{\mu}\right)\right| B\left(p_{B}\right)\right\rangle \equiv & l \epsilon_{\mu}+\left(\epsilon \cdot p_{B}\right)\left[c_{+}\left(p_{B}+p_{A}\right)_{\mu}+c_{-}\left(p_{B}-p_{A}\right)_{\mu}\right] \\
& -i q \epsilon_{\mu \nu \alpha \beta} \epsilon^{\nu}\left(p_{B}+p_{A}\right)^{\alpha}\left(p_{B}-p_{A}\right)^{\beta},
\end{aligned}
$$

where $A\left(p_{A}, \epsilon\right)$ is a ${ }^{3} P_{1}$ axial-vector meson. For the ${ }^{1} P_{1}$ axial-vector meson we change in the above matrix element $l$, $c_{+}, c_{-}$and $q$ by $r, s_{+}, s_{-}$and $v$, respectively.

Considering $B \rightarrow A$ transitions, at quark level $b \rightarrow q_{1}$, axial-vector meson $A$ has the quark content $q_{1} \bar{q}_{2}$, being $q_{2}$ the spectator quark. Thus, form factors defined in the ISGW2 model have the following expressions

$$
\begin{aligned}
l & =-\tilde{m}_{B} \beta_{B}\left[\frac{1}{\mu_{-}}+\frac{m_{2} \tilde{m}_{A}(\tilde{\omega}-1)}{\beta_{B}^{2}}\left(\frac{5+\tilde{\omega}}{6 m_{1}}-\frac{1}{2 \mu_{-}} \frac{m_{2}}{\tilde{m}_{A}} \frac{\beta_{B}^{2}}{\beta_{B A}^{2}}\right)\right] F_{5}^{(l)} \\
c_{+}+c_{-} & =-\frac{m_{2} \tilde{m}_{A}}{2 m_{1} \tilde{m}_{B} \beta_{B}}\left(1-\frac{m_{1} m_{2}}{2 \tilde{m}_{A} \mu_{-}} \frac{\beta_{B}^{2}}{\beta_{B A}^{2}}\right) F_{5}^{\left(c_{+}+c_{-}\right)} \\
c_{+}-c_{-} & =-\frac{m_{2} \tilde{m}_{A}}{2 m_{1} \tilde{m}_{B} \beta_{B}}\left(\frac{\tilde{\omega}+2}{3}-\frac{m_{1} m_{2}}{2 \tilde{m}_{A} \mu_{-}} \frac{\beta_{B}^{2}}{\beta_{B A}^{2}}\right) F_{5}^{\left(c_{+}-c_{-}\right)} \\
q & =-\frac{m_{2}}{2 \tilde{m}_{A} \beta_{B}}\left(\frac{5+\tilde{\omega}}{6}\right) F_{5}^{(q)}
\end{aligned}
$$

for the ${ }^{3} P_{1}$ axial-vector meson and

$$
\begin{aligned}
r & =\frac{\tilde{m}_{B} \beta_{B}}{\sqrt{2}}\left[\frac{1}{\mu_{+}}+\frac{m_{2} \tilde{m}_{A}}{3 m_{1} \beta_{B}^{2}}(\tilde{\omega}-1)^{2}\right] F_{5}^{(r)} \\
s_{+}+s_{-} & =\frac{m_{2}}{\sqrt{2} \tilde{m}_{B} \beta_{B}}\left(1-\frac{m_{2}}{m_{1}}+\frac{m_{2}}{2 \mu_{+}} \frac{\beta_{B}^{2}}{\beta_{B A}^{2}}\right) F_{5}^{\left(s_{+}+s_{-}\right)} \\
s_{+}-s_{-} & =\frac{m_{2}}{\sqrt{2} m_{1} \beta_{B}}\left(\frac{4-\tilde{\omega}}{3}-\frac{m_{1} m_{2}}{2 \tilde{m}_{A} \mu_{+}} \frac{\beta_{B}^{2}}{\beta_{B A}^{2}}\right) F_{5}^{\left(s_{+}-s_{-}\right)} \\
v & =\left[\frac{\tilde{m}_{B} \beta_{B}}{4 \sqrt{2} m_{b} m_{1} \tilde{m}_{A}}+\frac{(\tilde{\omega}-1)}{6 \sqrt{2}} \frac{m_{2}}{\tilde{m}_{A} \beta_{B}}\right] F_{5}^{(v)}
\end{aligned}
$$

for the ${ }^{1} P_{1}$ axial-vector meson. The $F_{5}^{(i)}$ factors in the above expressions are defined by 


$$
\begin{gathered}
F_{5}^{(l)}=F_{5}^{(r)}=F_{5}\left(\frac{\bar{m}_{B}}{\tilde{m}_{B}}\right)^{1 / 2}\left(\frac{\bar{m}_{A}}{\tilde{m}_{A}}\right)^{1 / 2}, \\
F_{5}^{(q)}=F_{5}^{(v)}=F_{5}\left(\frac{\bar{m}_{B}}{\tilde{m}_{B}}\right)^{-1 / 2}\left(\frac{\bar{m}_{A}}{\tilde{m}_{A}}\right)^{-1 / 2}, \\
F_{5}^{\left(c_{+}+c_{-}\right)}=F_{5}^{\left(s_{+}+s_{-}\right)}=F_{5}\left(\frac{\bar{m}_{B}}{\tilde{m}_{B}}\right)^{-3 / 2}\left(\frac{\bar{m}_{A}}{\tilde{m}_{A}}\right)^{1 / 2}, \\
F_{5}^{\left(c_{+}-c_{-}\right)}=F_{5}^{\left(s_{+}-s_{-}\right)}=F_{5}\left(\frac{\bar{m}_{B}}{\tilde{m}_{B}}\right)^{-1 / 2}\left(\frac{\bar{m}_{A}}{\tilde{m}_{A}}\right)^{-1 / 2},
\end{gathered}
$$

and the $F_{n}$ function by

$$
F_{n}=\left(\frac{\tilde{m}_{A}}{\tilde{m}_{B}}\right)^{1 / 2}\left(\frac{\beta_{B} \beta_{A}}{\beta_{B A}}\right)^{n / 2}\left[1+\frac{1}{18} r^{2}\left(t_{m}-t\right)\right]^{-3}
$$

where

$$
r^{2}=\frac{3}{4 m_{b} m_{1}}+\frac{3 m_{2}^{2}}{2 \bar{m}_{B} \bar{m}_{A} \beta_{B A}^{2}}+\frac{1}{\bar{m}_{B} \bar{m}_{A}}\left(\frac{16}{33-2 n_{f}}\right) \ln \left[\frac{\alpha_{s}\left(\mu_{\mathrm{QM}}\right)}{\alpha_{s}\left(m_{1}\right)}\right] .
$$

The parameters $m_{1}$ and $m_{2}$ are masses of quarks $q_{1}$ and $q_{2}, \bar{m}$ is the hyperfine averaged mass, $\tilde{m}$ is the sum of the masses of constituent quarks, $t_{m}=\left(m_{B}-m_{A}\right)^{2}$ is the maximum momentum transferred, $n_{f}$ is the number of active flavors at the $b$ scale and $\alpha_{s}(\mu)$ is the QCD coupling at the $\mu$ scale. The parameters $\beta_{B}, \beta_{A}$ are obtained from the model, see Ref. [11]. Moreover, we use the definitions

$$
\mu_{ \pm}=\left(\frac{1}{m_{1}} \pm \frac{1}{m_{b}}\right), \tilde{\omega}=\frac{t_{m}-t}{2 \bar{m}_{B} \bar{m}_{A}}+1
$$

and $\beta_{B A}^{2}=1 / 2\left(\beta_{B}^{2}+\beta_{A}^{2}\right)$.

In Table IV, we list values of form factors at momentum transferred $t=m_{\pi}^{2}$. Form factors are functions of momentum transferred $t=\left(p_{B}-p_{A}\right)^{2}$, see Eqs. (21) and (22). In general, form factors vary from $m_{\pi}^{2}$ to $m_{K_{1}(1400)}^{2}$, in just only $4 \%$. In addition, values for the form factors depend strongly on the parameters $\beta_{B}=0.43$ and $\beta=0.28$ calculated in the model.

We calculate form factors for $B \rightarrow K_{1 A}$ and $B \rightarrow K_{1 B}$ transitions in $S U(3)$ base. Branching ratios are calculated with physical form factors, which are obtained from the mixing, see Eq. (1).

TABLE IV. Form factors at momentum transfer $t=m_{\pi}^{2}$ for $B \rightarrow A$ transitions, evaluated in the ISGW2 model [1].

\begin{tabular}{lcccc}
\hline \hline Transition & $q$ & $l$ & $c_{+}$ & $c_{-}$ \\
\hline$B \rightarrow a_{1}$ & -0.0417 & -1.7469 & -0.0101 & -0.0012 \\
$B \rightarrow f_{1}$ & -0.0427 & -1.7603 & -0.0103 & -0.0012 \\
$B \rightarrow K_{1 A}$ & -0.0593 & -1.8567 & -0.0155 & -0.0011 \\
\multicolumn{5}{c}{} \\
$\quad$ & $r$ & $s_{+}$ & $s_{-}$ \\
$B \rightarrow b_{1}$ & 0.0319 & 0.9404 & 0.0177 & -0.0082 \\
$B \rightarrow h_{1}$ & 0.0324 & 0.9214 & 0.0134 & -0.0051 \\
$B \rightarrow K_{1 B}$ & 0.0323 & 0.8956 & 0.0275 & -0.0124 \\
\hline \hline
\end{tabular}

To compare form factor values for $B \rightarrow A$ transitions with those of $B \rightarrow V$ transitions, we can define $B \rightarrow A$ transitions in same basis as the used in the BSW model, see Eq. (17). We change the symbols for the form factors $V$ and $A_{0,1,2}$ by $A$ and $V_{0,1,2}$, respectively. These form factors are related to form factors in the ISGW2 model by

$$
\begin{aligned}
A\left(q^{\prime 2}\right)= & -\left(m_{B}+m_{A}\right) q\left(q^{\prime 2}\right), \quad V_{1}\left(q^{\prime 2}\right)=\frac{l\left(q^{\prime 2}\right)}{\left(m_{B}+m_{A}\right)}, \quad V_{2}\left(q^{\prime 2}\right)=-\left(m_{B}+m_{A}\right) c_{+}\left(q^{\prime 2}\right) \\
& V_{0}\left(q^{\prime 2}\right)=\frac{1}{2 m_{A}}\left[l\left(q^{\prime 2}\right)+\left(m_{B}^{2}-m_{A}^{2}\right) c_{+}\left(q^{\prime 2}\right)+q^{\prime 2} c_{-}\left(q^{\prime 2}\right)\right] .
\end{aligned}
$$


In Table $\mathrm{V}$, we show form factor values for $B \rightarrow A$ transitions at momentum transferred $t=m_{\pi}^{2}$, which correspond to those of Table IV.

TABLE V. Form factors $V_{0,1,2,3}$ and $A$ at momentum transfer $t=m_{\pi}^{2}$ for $B \rightarrow A$ transitions, evaluated in the ISGW2 model [11].

\begin{tabular}{lcccc}
\hline \hline Transition & $A$ & $V_{1}$ & $V_{2}$ & $V_{3}=V_{0}$ \\
\hline$B \rightarrow a_{1}$ & 0.271 & -0.268 & 0.068 & -0.818 \\
$B \rightarrow f_{1}$ & 0.280 & -0.268 & 0.068 & -0.792 \\
$B \rightarrow K_{1 A}$ & 0.389 & -0.283 & 0.102 & -0.890 \\
$B \rightarrow b_{1}$ & -0.208 & 0.145 & -0.115 & 0.572 \\
$B \rightarrow h_{1}$ & -0.209 & 0.143 & -0.086 & 0.546 \\
$B \rightarrow K_{1 B}$ & -0.216 & 0.134 & -0.184 & 0.573 \\
\hline \hline
\end{tabular}

\section{AMPLITUDES AND BRANCHING RATIOS}

Let us present a comparison between $B \rightarrow V$ and $B \rightarrow A$ transitions, which seems straightforward. First, we can see, from sections 2, 4, and 6 in appendix B in Ref. [7], that parametrizations of $\left\langle V\left|J_{\mu}\right| B\right\rangle$ and $\left\langle A\left|J_{\mu}\right| B\right\rangle$ are only different by a global sign, with the substitution of the form factors $f \leftrightarrow l, r, a_{ \pm} \leftrightarrow c_{ \pm}, s_{ \pm}, g \leftrightarrow q, v$. This is because behavior of currents $V_{\mu}$ and $A_{\mu}$ are interchanged. Moreover, this implies that expressions for decay amplitudes and decay rates, at tree level, for the processes $B \rightarrow V P, V V$ and $B \rightarrow A P, A V$, and $A A$, respectively, are identical.

On the other hand, the situation is different when tree and penguin contributions are considered. The expressions of decay amplitudes for processes $B \rightarrow A P$ (see appendix A) and $B \rightarrow V P$ (see appendices in Refs. 3, 4]) are equal when only QCD parameters $a_{3}, a_{4}, a_{9}$ and $a_{10}$ contribute. When QCD parameters $a_{6}$ and $a_{8}$ contribute then the linear combination $\left(z a_{6}+y a_{8}\right)$ is affected by a global sign and $1 /\left(m_{b}-m_{q}\right)$, which is a factor of this linear combination, changes by $1 /\left(m_{b}+m_{q}\right)$. Relevant contributions in penguin sector are coefficients $a_{4}$ and $a_{6}$ (see appendix A); in $B \rightarrow A$ transitions $a_{6}$ and $a_{4}$ have same sign. This fact implies that these terms are summed so their contribution increase. In $B \rightarrow V$ transitions, these terms have different sign thus their contribution decrease. The contributions corresponding to $a_{5}$ and $a_{7}$ change sign when the axial-vector or the vector meson arises from vacuum, but they are not affected if the pseudoscalar meson is produced from vacuum.

Now we are going to compare penguin contributions to decay amplitudes $\mathcal{M}(B \rightarrow A V)$ (see appendix B) with the ones $\mathcal{M}(B \rightarrow V V)$ (showed in appendices $\mathrm{F}$ and $\mathrm{G}$ in Ref. [4]): (i) in both cases contribution of parameters $a_{6}$ and $a_{8}$ does not appear; (ii) sign of contribution given by parameters $a_{5}$ and $a_{7}$ changes when one goes from $B \rightarrow V, A$ to $B \rightarrow V, V$; (iii) in modes $B \rightarrow V, V^{\text {charged }}$ and $B \rightarrow V, A^{\text {charged }}$ always appears the contribution $\left(a_{4}+a_{10}\right)$.

In Table VI, we have summarized penguin contributions to decay amplitudes for modes $B \rightarrow A P$ displayed in appendix A, without including $P=\eta^{\left({ }^{\prime}\right)}$. These decay amplitudes can be classified in two groups from these contributions. The first group is integrated by decays where a charged meson in final state is produced from vacuum and penguin contribution is given by the linear combination $\left(a_{4}+a_{10}\right)+\alpha\left(a_{6}+a_{8}\right) R$, i.e., parameters $a_{\text {even }}$ only contribute to this group. Additionally, in this group we find two cases with $\alpha=0$ and $\alpha=1$, which correspond to modes $B \rightarrow P, A^{\text {charged }}$ and $B \rightarrow A, P^{\text {charged }}$, respectively. Here the notation $B \rightarrow M_{1}, M_{2}$ means that meson $M_{2}$ can be factorized out under the factorization approximation.

The second group is integrated by decays where a neutral meson is factorized out under factorization approximation independently if it is pseudoscalar or axial-vector. Penguin contribution is given by the linear combination $\alpha_{1}\left(a_{4}-\right.$ $\left.a_{10} / 2\right)+\alpha_{2}\left(a_{6}-a_{8} / 2\right) R+\alpha_{3}\left(a_{7}-a_{9}\right)+\alpha_{4}\left(a_{3}-a_{5}\right)$. Pure penguin contributions belong to this group and have contributions of $a_{\text {even }}$. They arise when the axial-vector meson or the pseudoscalar meson is a neutral strange meson and, of course, it is produced from vacuum. QCD parameters $a_{4}, a_{6}, a_{8}$ and $a_{10}$ contribute when the pseudoscalar $K^{0}$ meson is factorized out under factorization approximation, $a_{4}$ and $a_{10}$ when the axial-vector $K_{1}^{0}$ meson arises from vacuum. Note, that in general, decays $B \rightarrow P, A$ do not have contributions from $a_{6}$ and $a_{8}$.

In Table VII, we have classified penguin contributions to decay amplitudes $\mathcal{M}(B \rightarrow A V)$ which are showed in appendix B. There are two types: in one of them the linear combination $\alpha_{1}\left(a_{4}+a_{10}\right)+\alpha_{2}\left(a_{7} \pm a_{9}\right)$ contribute. It occurs when decays $B \rightarrow A, V^{\text {charged }}$ or $B \rightarrow V, A^{\text {charged }}$ are produced, i.e., when a charged meson in the final state arises from vacuum; in the other case, a neutral meson is factorized out under factorization approximation and the linear combination $\beta_{1}\left(a_{4}-a_{10} / 2\right)+\beta_{2}\left(a_{3} \pm a_{5}\right)+\beta_{3}\left(a_{7} \pm a_{9}\right)$ contributes. Pure penguin contributions belong to it. Parameters $a_{\text {odd }}$ contribute to decay amplitude of pure penguin modes $\bar{B}^{0} \rightarrow a_{1}^{0} f_{1}$ and $\bar{B}^{0} \rightarrow a_{1}^{0} \phi$. Like decays $B \rightarrow P, A$, in general, decays $B \rightarrow A V$ do not have contributions from $a_{6}$ and $a_{8}$.

Penguin contributions of decay amplitudes $\mathcal{M}(B \rightarrow A A)$ (see appendix $\mathrm{C}$ ) can be classified in a similar way. There are two groups. In one of them a charged meson is factorized out under factorization scheme and only $a_{4}$ and $a_{10}$ 
parameters contribute by means of the linear combination $\left(a_{4}+a_{10}\right)$. In the other group a neutral meson is produced from vacuum. In this case the linear combination $\zeta_{1}\left(a_{4}-a_{10} / 2\right)+\zeta_{2}\left(a_{3}-a_{5}\right)+\zeta_{3}\left(a_{7}-a_{9}\right)$ contribute. In Table VIII, we display the respective coefficients $\zeta_{i}$. Again, pure penguin decays are in this group.

TABLE VI. Coefficients of the linear combinations $\left(a_{4}+a_{10}\right)+\alpha\left(a_{6}+a_{8}\right) R$ and $\alpha_{1}\left(a_{4}-a_{10} / 2\right)+\alpha_{2}\left(a_{6}-a_{8} / 2\right) R+\alpha_{3}\left(a_{7}-a_{9}\right)+\alpha_{4}\left(a_{3}-a_{5}\right)$ corresponding to penguin contribution of decay amplitudes $\mathcal{M}(B \rightarrow A P)$ without $P=\eta^{\left({ }^{\circ}\right)}$. The coefficient $\mathrm{R}$ is given by $R=2 m_{P}^{2} /\left(m_{1}+m_{2}\right)\left(m_{b}-m_{3}\right)$.

\begin{tabular}{|c|c|c|c|c|}
\hline Decays & $\alpha \alpha_{1}$ & $\alpha_{2}$ & $\overline{\alpha \alpha_{3}}$ & $\overline{\alpha_{4}}$ \\
\hline $\begin{array}{l}\bar{B}^{0} \rightarrow \pi^{+}, a_{1}^{-} ; B^{-} \rightarrow \pi^{0}, a_{1}^{-} ; \bar{B}^{0} \rightarrow \pi^{+}, K_{1}^{-} ; B^{-} \rightarrow \pi^{0}, K_{1}^{-} \\
\bar{B}^{0} \rightarrow a_{1}^{+}, \pi^{-}: B^{-} \rightarrow a_{1}^{0}, \pi^{-}: \bar{B}^{0} \rightarrow a_{1}^{+}, K^{-}: B^{-} \rightarrow a_{1}^{0}, K^{-}: B^{-} \rightarrow f_{1}, \pi^{-}: B^{-} \rightarrow f_{1}, K^{-}\end{array}$ & $\begin{array}{ll}0 \\
1\end{array}$ & & & \\
\hline $\bar{B}^{0} \rightarrow \pi^{0}, f_{1} ; B^{-} \rightarrow \pi^{-}, f_{1}$ & 1 & 0 & $-1 / 2$ & 2 \\
\hline $\bar{B}^{0} \rightarrow a_{1}^{0}, \pi^{0} ; B^{-} \rightarrow a_{1}^{-}, \pi^{0} ; \bar{B}^{0} \rightarrow f_{1}, \pi^{0}$ & \pm 1 & \pm 1 & $\pm 3 / 2$ & 0 \\
\hline $\bar{B}^{0} \rightarrow \pi^{0}, a_{1}^{0} ; B^{-} \rightarrow \pi^{-}, a_{1}^{0}$ & -1 & 0 & $-3 / 2$ & 0 \\
\hline $\bar{B}^{0} \rightarrow \bar{K}^{0}, f_{1} ; B^{-} \rightarrow K^{-}, f_{1}$ & 0 & 0 & $-1 / 2$ & 2 \\
\hline $\bar{B}^{0} \rightarrow f_{1}, \bar{K}^{0} ; B^{-} \rightarrow a_{1}^{-}, \bar{K}^{0} ; \bar{B}^{0} \rightarrow a_{1}^{0}, \bar{K}^{0} ; \bar{B}^{0} \rightarrow \bar{K}_{1}^{0}, K^{0} ; B^{-} \rightarrow K_{1}^{-}, K^{0}$ & 1 & 1 & 0 & 0 \\
\hline $\bar{B}^{0} \rightarrow \bar{K}^{0}, a_{1}^{0} ; B^{-} \rightarrow K^{-}, a_{1}^{0} ; B^{-} \rightarrow K_{1}^{-}, \pi^{0} ; \bar{B}^{0} \rightarrow \bar{K}_{1}^{0}, \pi^{0}$ & 0 & 0 & $-3 / 2$ & 0 \\
\hline $\bar{B}^{0} \rightarrow \pi^{0}, K_{1}^{0} ; B^{-} \rightarrow \pi^{-}, \bar{K}_{1}^{0} ; \bar{B}^{0} \rightarrow \bar{K}^{0}, K_{1}^{0} ; B^{-} \rightarrow K^{-}, K_{1}^{0}$ & 1 & 0 & 0 & 0 \\
\hline
\end{tabular}

TABLE VII. Coefficients of the linear combinations $\alpha_{1}\left(a_{4}+a_{10}\right)+\alpha_{2}\left(a_{7} \pm a_{9}\right)$ and $\beta_{1}\left(a_{4}-a_{10} / 2\right)+\beta_{2}\left(a_{3} \pm a_{5}\right)+\beta_{3}\left(a_{7} \pm a_{9}\right)$ corresponding to penguin contribution of decay amplitudes $\mathcal{M}(B \rightarrow A V)$.

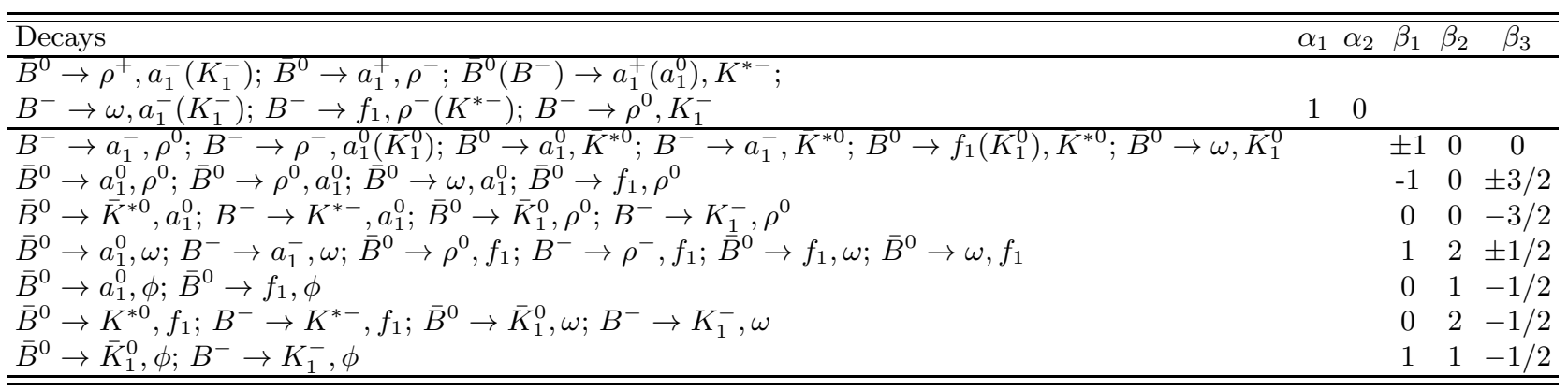

TABLE VIII. Coefficients of the linear combination $\zeta_{1}\left(a_{4}-a_{10} / 2\right)+\zeta_{2}\left(a_{3}-a_{5}\right)+\zeta_{3}\left(a_{7}-a_{9}\right)$ corresponding to penguin contribution of decay amplitudes $\mathcal{M}\left(B \rightarrow A, A^{\text {neutral }}\right)$.

\begin{tabular}{lccc}
\hline \hline Decays & $\zeta_{1} \zeta_{2}$ & $\zeta_{3}$ \\
\hline $\bar{B}^{0} \rightarrow a_{1}^{0}, a_{1}^{0} ; \bar{B}^{0} \rightarrow f_{1}, a_{1}^{0}$ & -1 & 0 & $-3 / 2$ \\
$\bar{B}^{0} \rightarrow a_{1}^{0}, f_{1} ; B^{-} \rightarrow a_{1}^{-}, f_{1}$ & 1 & 2 & $-1 / 2$ \\
$\bar{B}^{0} \rightarrow a_{1}^{0}, \bar{K}_{1}^{0} ; B^{-} \rightarrow a_{1}^{-}, \bar{K}_{1}^{0} ; \bar{B}^{0} \rightarrow f_{1}, \bar{K}_{1}^{0}$ & 1 & 0 & 0 \\
$\bar{B}^{0} \rightarrow \bar{K}_{1}^{0}, a_{1}^{0} ; B^{-} \rightarrow K_{1}^{-}, a_{1}^{0}$ & 0 & 0 & $-3 / 2$ \\
$\bar{B}^{0} \rightarrow \bar{K}_{1}^{0}, f_{1} ; B^{-} \rightarrow K_{1}^{-}, f_{1}$ & 0 & 2 & $-1 / 2$ \\
\hline \hline
\end{tabular}

In appendices we give explicitly the amplitudes to the processes studied in terms of form factors for $B \rightarrow P, B \rightarrow V$ and $B \rightarrow A$ transitions. In appendix $\mathrm{A}$ we have a common factor $\left(\epsilon^{*} \cdot p_{B}\right)$ which is not included in the expressions to simplify. And we use the symbol $K_{1}$ to indicate the axial-vector mesons $K_{1}(1270)$ or $K_{1}(1400)$. In appendices we have the factor $G_{F} / \sqrt{2}$ common to all amplitudes.

It is straightforward to calculate the branching ratios from amplitudes and input parameters. However, here we give general expressions which are useful in decay rates estimations. The decay rate formula for $B \rightarrow A X$ decays is, in general, given by

$$
\Gamma(B \rightarrow A X)=\frac{p_{c}}{8 \pi m_{B}^{2}}|\mathcal{M}(B \rightarrow A X)|^{2}
$$

where $p_{c}=\lambda^{1 / 2}\left(m_{B}^{2}, m_{A}^{2}, m_{V}^{2}\right) / 2 m_{B}$ is the momentum of decay particle in the rest frame of $B$ meson and $X$ can be $P, V$ or $A$ and $\lambda(a, b, c) \equiv a^{2}+b^{2}+c^{2}-2(a b+a c+b c)$ 
For branching ratios of $B \rightarrow A P$ decays, we note that amplitude $\mathcal{M}(B \rightarrow A P)$ is proportional to $\left(\epsilon_{A}^{*} \cdot p_{B}\right)$. Thus amplitude squared is proportional to $\left|\left(\varepsilon_{A}^{*} \cdot p_{B}\right)\right|^{2}$, which is easily calculated. The general decay rate formula for $B \rightarrow A V$ decays is more involved, because the amplitude $\mathcal{M}(B \rightarrow A V)$ includes an interfering term. Since the long expressions we do not write here.

\section{NUMERICAL RESULTS}

In this section we present our numerical results. In Tables IX-X, XI-XII, and XIII-XIV we display branching ratios of $B \rightarrow A P, B \rightarrow A V$ and $B \rightarrow A A$ decays, respectively, using the improved version of the ISGW quark model [1] for calculating form factors for $B \rightarrow A$ transitions.

Branching ratios for $B \rightarrow A P$ decays, where $A$ is a ${ }^{3} P_{1}$ nonstrange axial-vector meson (see Table IX) are bigger than ones where $A$ is a ${ }^{1} P_{1}$ nonstrange axial-vector meson. The ratio $B r\left(B \rightarrow A\left({ }^{3} P_{1}\right) P\right) / B r\left(B \rightarrow A\left({ }^{1} P_{1}\right) P\right)$, where mesons $A\left({ }^{3} P_{1}\right)$ and $A\left({ }^{1} P_{1}\right)$ have the same quark content, is $\sim 1.6-4.5$, except for a small number of them. The mode $B^{-} \rightarrow a_{1}^{-} \bar{K}^{0}$, which is a pure penguin channel, is the most dominant (its branching ratio of $84.1 \times 10^{-6}$ is the biggest). Penguin contribution to this mode is given by $a_{\text {even }}$ parameters. Other dominant decays are $\bar{B}^{0} \rightarrow a_{1}^{+} \pi^{-}$and $\bar{B}^{0} \rightarrow a_{1}^{+} K^{-}$, whose branching ratios are $74.3 \times 10^{-6}$ and $72.2 \times 10^{-6}$, respectively. In these decays there is a destructive interference between penguin and $W$-external or $W$-internal contributions. On the other hand, a similar situation is found changing a ${ }^{3} P_{1}$ meson by a ${ }^{1} P_{1}$ meson with the same quark content (see fourth column in Table IX). At experimental level there is not enough information. Our predictions for $\operatorname{Br}\left(\bar{B}^{0} \rightarrow a_{1}^{-} \pi^{+}\right)=36.7 \times 10^{-6}$ and $B r\left(\bar{B}^{0} \rightarrow\right.$ $\left.a_{1}^{+} \pi^{-}\right)=74.3 \times 10^{-6}$ are consistent with the experimental average value $\operatorname{Br}\left(\bar{B}^{0} \rightarrow a_{1}^{\mp} \pi^{ \pm}\right)=(40.9 \pm 7.6) \times 10^{-6}[8]$. This average includes BaBar and Belle results [1]. Finally, we want to mention that our predictions are at the same order that the ones obtained by Laporta-Nardulli-Pham (see Tables V and VI in Ref. [8]), although our values are in general bigger, except in a few modes.

In Table $\mathrm{X}$, we show branching ratios for $B \rightarrow K_{1} P$ decays for two values $\left(\theta=32^{\circ}, 58^{\circ}\right)$ of the mixing angle $K_{A}-K_{B}$. The strange axial-vector meson is $K_{1}(1270)$ or $K_{1}(1400)$. In this case, the most dominant decays are $B^{-} \rightarrow K_{1}^{-}(1400) \eta^{\left({ }^{\prime}\right)}$, with branching ratios of $\mathcal{O}\left(10^{-5}\right)$. On the other hand, branching ratios of modes $\bar{B}^{0} \rightarrow$ $K_{1}^{-} \pi^{+}, \bar{B}^{0} \rightarrow \bar{K}_{1}^{0} \pi^{0}, B^{-} \rightarrow K_{1}^{-} \pi^{0}, B^{-} \rightarrow \bar{K}_{1}^{0} \pi^{-}, \bar{B}^{0} \rightarrow K_{1}^{0} \bar{K}^{0}$ and $B^{-} \rightarrow K_{1}^{0} K^{-}$, where the $K_{1}$ meson can be $K_{1}(1270)$ or $K_{1}(1400)$, are not sensitive to the value of the mixing angle. On the contrary, branching ratios of $\bar{B}^{0} \rightarrow \bar{K}_{1}^{0}(1270) \eta^{\left({ }^{\prime}\right)}\left(K^{0}\right)$ and $B^{-} \rightarrow K_{1}^{-}(1270) \eta^{\left({ }^{\prime}\right)}\left(K^{0}\right)$ strongly depend on the mixing angle. The same decays but changing $K_{1}(1270)$ by $K_{1}(1400)$ are not very sensitive to this angle. Branching ratios of $B \rightarrow K_{1}(1270) P$ and $B \rightarrow K_{1}(1400) P$ are smaller with $\theta=32^{\circ}$ and $\theta=58^{\circ}$, respectively. Laporta-Nardulli-Pham, in Table IV, Ref. [8], displayed some of the branching ratios that we present in Table X. In general, both predictions agree.

In Table XI, we display branching ratios for $B \rightarrow A V$ decays with $A$ being a ${ }^{3} P_{1}$ or a ${ }^{1} P_{1}$ nonstrange axial-vector meson. The most of these decays are suppressed. In general, $B r\left(B \rightarrow A\left({ }^{3} P_{1}\right) V\right)$ is bigger than $B r\left(B \rightarrow A\left({ }^{1} P_{1}\right) V\right)$, where mesons $A\left({ }^{3} P_{1}\right)$ and $A\left({ }^{1} P_{1}\right)$ have the same quark content. In this case, dominant decays are $B^{-} \rightarrow f_{1} \rho^{-}, \bar{B}^{0} \rightarrow$ $a_{1}^{ \pm} \rho^{\mp}$. Their branching ratios are $\mathcal{O}\left(10^{-6}\right)$. If we compare Tables IX and XI we found that $B r\left(B \rightarrow A P\left(q_{1} \bar{q}_{2}\right)\right)>$ $\operatorname{Br}\left(B \rightarrow A V\left(q_{1} \bar{q}_{2}\right)\right)$.

In Table XII, we present branching ratios for $B \rightarrow K_{1} V$ decays for two values $\left(\theta=32^{\circ}, 58^{\circ}\right)$ of the mixing angle $K_{A}-K_{B}$. The strange axial-vector meson is $K_{1}(1270)$ or $K_{1}(1400)$. These decays are, in general, suppressed. The dominant decays are $\bar{B}^{0} \rightarrow \bar{K}_{1}^{0}(1400) K^{* 0}, B^{-} \rightarrow K_{1}^{-}(1400) K^{* 0}$ and $B^{-} \rightarrow K_{1}^{0}(1270) K^{*-}$. Their branching ratios are $\mathcal{O}\left(10^{-6}\right)$. On the other hand, the branching ratios of modes $\bar{B}^{0} \rightarrow K_{1}^{-} \rho^{+}, B^{-} \rightarrow \bar{K}_{1}^{0} \rho^{-}, B^{-} \rightarrow K_{1}^{-} \omega, \bar{B}^{0} \rightarrow K_{1}^{0} \bar{K}^{* 0}$ and $B^{-} \rightarrow K_{1}^{0} K^{*-}$, with $K_{1}=K_{1}(1270), K_{1}(1400)$, are not sensitive to the value of the mixing angle. On the contrary, branching ratio of $\bar{B}^{0} \rightarrow \bar{K}_{1}^{0}(1270) K^{* 0}$ and $B^{-} \rightarrow K_{1}^{-}(1270) K^{* 0}$ strongly depend on the value of $\theta$. The predictions obtained in Ref. [9] (see Tables II and III) for $B \rightarrow K_{1}(1270) \phi$ and $B \rightarrow K_{1}(1400) \phi$ and our predictions for these modes do not agree, except for the case with $N_{c}^{e f f}=\infty$ and $\theta=58^{\circ}$ (with $\mu=2.5 \mathrm{GeV}$ or $\mu=4.4 \mathrm{GeV}$ ). In this case the respective branching ratios are $\mathcal{O}\left(10^{-7}\right)$.

The branching ratios for $B \rightarrow b_{1}\left(h_{1}\right) P$ and $B \rightarrow b_{1}\left(h_{1}\right) V$ decays are calculated in the $S U(3)$ symmetry limit, i.e. $f_{b_{1}}=f_{h_{1}}=0$. In Table XIII we include the modes which change values with respect to Tables IX and XI. The branching ratios for $\bar{B}^{0} \rightarrow b_{1}^{-} \pi^{+}$and $\bar{B}^{0} \rightarrow b_{1}^{-} \rho^{+}$decays are zero since the amplitude is proportional to the decay constant $f_{b_{1}}$. The branching ratios for $B^{-} \rightarrow b_{1}^{-} \pi^{0}, B^{-} \rightarrow b_{1}^{-} \eta^{\left({ }^{\prime}\right)}, B^{-} \rightarrow b_{1}^{-} \omega$ decays decrease by one order of magnitude and for $B^{-} \rightarrow h_{1} K^{*-}, \bar{B}^{0} \rightarrow h_{1} K^{* 0}$ decays decrease by one half order of magnitude with respect to the values in Tables IX and XI. The modes $B^{-} \rightarrow b_{1}^{-} \eta^{\prime}, B^{-} \rightarrow b_{1}^{0} \omega$ and $\bar{B}^{0} \rightarrow h_{1} \rho^{0}$ increase by one order of magnitude.

Moreover, in Tables IX-XII, between brackets we show values corresponding to the branching ratios $B \rightarrow A P$ and $B \rightarrow A V$, where $B \rightarrow P(V)$ transitions are calculated in the LCSR approach. The values with the symbol [-] represent branching ratios which basically have equal value with respect to the calculated with the WSB model. In general, the branching ratios are smaller compared with the calculated with the BSW model. The branching ratios 
for $B^{-} \rightarrow a_{1}^{-} \omega, B^{-} \rightarrow b_{1}^{-} \omega$ and $B \rightarrow K_{1} \omega$ decays increase their values, because in the LCSR approach the form factors for $B \rightarrow \omega$ transitions are bigger compared with the BSW model.

In Table XIV, we present branching ratios for five $B \rightarrow A A$ decays, where $A$ is a nonstrange axial-vector meson. The branching ratio of $\bar{B}^{0} \rightarrow a_{1}^{-} a_{1}^{+}$is $\mathcal{O}\left(10^{-6}\right)$. In this group, this decay is dominant. From Tables XI (see second column) and XIV we conclude that $\operatorname{Br}\left(B \rightarrow a_{1}^{-} V\left(q_{1} \bar{q}_{2}\right) \sim \operatorname{Br}\left(B \rightarrow a_{1}^{-} A\left(q_{1} \bar{q}_{2}\right)\right)\right.$, where $V$ and $A$ are nonstrange mesons.

In Table XV, we show branching ratios for $B \rightarrow K_{1} A$ decays for two values $\left(\theta=32^{\circ}, 58^{\circ}\right)$ of the mixing angle $K_{A}-K_{B}$. The strange axial-vector meson is $K_{1}(1270)$ or $K_{1}(1400)$. Branching ratios of the decays $\bar{B}^{0} \rightarrow K_{1}^{-} a_{1}^{+}$ and $B^{-} \rightarrow \bar{K}_{1}^{0} a_{1}^{-}$are not sensitive to the mixing angle. In this group, $\operatorname{Br}\left(\bar{B}^{0} \rightarrow K_{1}^{-}(1270) a_{1}^{+}\right) \sim 10^{-7}$ is the biggest. From Tables XII and XV we conclude that $\operatorname{Br}\left(B \rightarrow K_{1} \rho\right) \sim \operatorname{Br}\left(B \rightarrow K_{1} a_{1}\right)$.

Finally, in Table XVI, we present a summary about experimental information given in Ref. [5] for branching ratios of some charmless $B \rightarrow A P, A V, A A$ decays. In general, bounds for these branching ratios are $<\left(10^{-3}-10^{-4}\right)$. There is a similar situation for charmed and charmonium $B$ decays [5].

TABLE IX. Branching ratios (in units of $10^{-6}$ ) of $B \rightarrow A P$ decays, where $A$ is a nonstrange axial-vector meson, using the ISGW2 form factors for $B \rightarrow A$ transitions and BSW [LCSR] for $B \rightarrow P$ transitions.

\begin{tabular}{lclc}
\hline \hline Mode & \multicolumn{1}{c}{$\mathcal{B}$} & Mode & $\mathcal{B}$ \\
\hline $\bar{B}^{0} \rightarrow a_{1}^{-} \pi^{+}$ & $36.7[23.5]$ & $B^{0} \rightarrow b_{1}^{-} \pi^{+}$ & $4.4[2.8]$ \\
$\bar{B}^{0} \rightarrow a_{1}^{+} \pi^{-}$ & $74.3[-]$ & $\bar{B}^{0} \rightarrow b_{1}^{+} \pi^{-}$ & $36.2[-]$ \\
$\bar{B}^{0} \rightarrow a_{1}^{0} \pi^{0}$ & $0.27[-]$ & $\bar{B}^{0} \rightarrow b_{1}^{0} \pi^{0}$ & $0.15[-]$ \\
$B^{-} \rightarrow a_{1}^{-} \pi^{0}$ & $13.6[7.8]$ & $B^{-} \rightarrow b_{1}^{-} \pi^{0}$ & $4.2[3.1]$ \\
$B^{-} \rightarrow a_{1}^{0} \pi^{-}$ & $43.2[-]$ & $B^{-} \rightarrow b_{1}^{0} \pi^{-}$ & $18.6[-]$ \\
$\bar{B}^{0} \rightarrow a_{1}^{0} \eta$ & $0.54[-]$ & $\bar{B}^{0} \rightarrow b_{1}^{0} \eta$ & $0.17[-]$ \\
$B^{-} \rightarrow a_{1}^{-} \eta$ & $13.4[9.1]$ & $B^{-} \rightarrow b_{1}^{-} \eta$ & $0.88[0.51]$ \\
$\bar{B}^{0} \rightarrow a_{1}^{0} \eta^{\prime}$ & $0.09[-]$ & $\bar{B}^{0} \rightarrow b_{1}^{0} \eta^{\prime}$ & $0.02[-]$ \\
$B^{-} \rightarrow a_{1}^{-} \eta^{\prime}$ & $13.6[10.1]$ & $B^{-} \rightarrow b_{1}^{-} \eta^{\prime}$ & $0.02[0.001]$ \\
$\bar{B}^{0} \rightarrow a_{1}^{0} \bar{K}^{0}$ & $42.3[-]$ & $\bar{B}^{0} \rightarrow b_{1}^{0} \bar{K}^{0}$ & $19.3[-]$ \\
$\bar{B}^{0} \rightarrow a_{1}^{+} K^{-}$ & $72.2[-]$ & $\bar{B}^{0} \rightarrow b_{1}^{+} K^{-}$ & $35.7[-]$ \\
$B^{-} \rightarrow a_{1}^{0} K^{-}$ & $43.4[-]$ & $B^{-} \rightarrow b_{1}^{0} K^{-}$ & $18.1[-]$ \\
$B^{-} \rightarrow a_{1}^{-} \bar{K}^{0}$ & $84.1[-]$ & $B^{-} \rightarrow b_{1}^{-} \bar{K}^{0}$ & $41.5[-]$ \\
$\bar{B}^{0} \rightarrow f_{1} \pi^{0}$ & $0.47[-]$ & $\bar{B}^{0} \rightarrow h_{1} \pi^{0}$ & $0.16[-]$ \\
$B^{-} \rightarrow f_{1} \pi^{-}$ & $34.1[-]$ & $B^{-} \rightarrow h_{1} \pi^{-}$ & $18.6[-]$ \\
$\bar{B}^{0} \rightarrow f_{1} \eta$ & $37.1[-]$ & $\bar{B}^{0} \rightarrow h_{1} \eta$ & $18.2[-]$ \\
$\bar{B}^{0} \rightarrow f_{1} \eta^{\prime}$ & $22.1[-]$ & $\bar{B}^{0} \rightarrow h_{1} \eta^{\prime}$ & $11.2[-]$ \\
$\bar{B}^{0} \rightarrow f_{1} \bar{K}^{0}$ & $34.7[-]$ & $\bar{B}^{0} \rightarrow h_{1} \bar{K}^{0}$ & $19.0[-]$ \\
$B^{-} \rightarrow f_{1} K^{-}$ & $31.1[-]$ & $B^{-} \rightarrow h_{1} K^{-}$ & $19.0[-]$ \\
\hline \hline
\end{tabular}

TABLE X. Branching ratios (in units of $10^{-6}$ ) of $B \rightarrow A P$ decays, where $A$ is a strange axial-vector meson $K_{1}(1270)$ or $K_{1}(1400)$, using the ISGW2 form factors for $B \rightarrow A$ transitions and BSW [LCSR] for $B \rightarrow P$ transitions.

\begin{tabular}{lcclcc}
\hline \hline Mode & $\mathcal{B}\left(32^{\circ}\right)$ & $\mathcal{B}\left(58^{\circ}\right)$ & Mode & $\mathcal{B}\left(32^{\circ}\right)$ & $\mathcal{B}\left(58^{\circ}\right)$ \\
\hline $\bar{B}^{0} \rightarrow K_{1}^{-}(1270) \pi^{+}$ & $4.3[2.8]$ & $4.3[2.8]$ & $\bar{B}^{0} \rightarrow K_{1}^{-}(1400) \pi^{+}$ & $2.3[1.5]$ & $2.3[1.5]$ \\
$\bar{B}^{0} \rightarrow \bar{K}_{1}^{0}(1270) \pi^{0}$ & $2.3[1.5]$ & $2.1[1.4]$ & $\bar{B}^{0} \rightarrow \bar{K}_{1}^{0}(1400) \pi^{0}$ & $1.7[1.3]$ & $1.6[1.3]$ \\
$B^{-} \rightarrow K_{1}^{-}(1270) \pi^{0}$ & $2.5[1.6]$ & $1.6[0.9]$ & $B^{-} \rightarrow K_{1}^{-}(1400) \pi^{0}$ & $0.67[0.51]$ & $0.64[0.55]$ \\
$B^{-} \rightarrow \bar{K}_{1}^{0}(1270) \pi^{-}$ & $4.7[3.0]$ & $4.7[3.0]$ & $B^{-} \rightarrow \bar{K}_{1}^{0}(1400) \pi^{-}$ & $2.5[1.7]$ & $2.5[1.7]$ \\
$\bar{B}^{0} \rightarrow \bar{K}_{1}^{0}(1270) \eta$ & $1.5[1.1]$ & $10.2[9.8]$ & $\bar{B}^{0} \rightarrow \bar{K}_{1}^{0}(1400) \eta$ & $52.8[52.5]$ & $46.8[46.6]$ \\
$B^{-} \rightarrow K_{1}^{-}(1270) \eta$ & $0.95[0.65]$ & $20.7[19.4]$ & $B^{-} \rightarrow K_{1}^{-}(1400) \eta$ & $95.1[93.3]$ & $84.8[83.1]$ \\
$\bar{B}^{0} \rightarrow \bar{K}_{1}^{0}(1270) \eta^{\prime}$ & $1.1[0.8]$ & $9.4[9.1]$ & $\bar{B}^{0} \rightarrow \bar{K}_{1}^{0}(1400) \eta^{\prime}$ & $51.4[51.2]$ & $46.0[45.8]$ \\
$B^{-} \rightarrow K_{1}^{-}(1270) \eta^{\prime}$ & $0.53[0.4]$ & $16.6[15.6]$ & $B^{-} \rightarrow K_{1}^{-}(1400) \eta^{\prime}$ & $80.0[78.5]$ & $71.9[70.5]$ \\
$\bar{B}^{0} \rightarrow K_{1}^{0}(1270) \bar{K}^{0}$ & $0.20[0.17]$ & $0.20[0.17]$ & $\bar{B}^{0} \rightarrow K_{1}^{0}(1400) \bar{K}^{0}$ & $0.11[0.09]$ & $0.11[0.09]$ \\
$\bar{B}^{0} \rightarrow \bar{K}_{1}^{0}(1270) K^{0}$ & $0.02[-]$ & $0.70[-]$ & $\bar{B}^{0} \rightarrow \bar{K}_{1}^{0}(1400) K^{0}$ & $4.1[-]$ & $3.6[-]$ \\
$B^{-} \rightarrow K_{1}^{-}(1270) K^{0}$ & $0.02[-]$ & $0.75[-]$ & $B^{-} \rightarrow K_{1}^{-}(1400) K^{0}$ & $4.4[-]$ & $3.9[-]$ \\
$B^{-} \rightarrow K_{1}^{0}(1270) K^{-}$ & $0.22[0.18]$ & $0.22[0.18]$ & $B^{-} \rightarrow K_{1}^{0}(1400) K^{-}$ & $0.12[0.10]$ & $0.12[0.10]$ \\
\hline \hline
\end{tabular}


TABLE XI. Branching ratios (in units of $10^{-6}$ ) of $B \rightarrow A V$ decays, where $A$ is a nonstrange axial-vector meson, using the ISGW2 form factors $B \rightarrow A$ transitions and BSW [LCSR] for $B \rightarrow V$ transitions.

\begin{tabular}{lclc}
\hline \hline Mode & $\mathcal{B}$ & Mode & $\mathcal{B}$ \\
\hline $\bar{B}^{0} \rightarrow a_{1}^{-} \rho^{+}$ & $4.7[3.5]$ & $\bar{B}^{0} \rightarrow b_{1}^{-} \rho^{+}$ & $0.55[0.41]$ \\
$\bar{B}^{0} \rightarrow a_{1}^{+} \rho^{-}$ & $4.3[-]$ & $\bar{B}^{0} \rightarrow b_{1}^{+} \rho^{-}$ & $1.6[-]$ \\
$\bar{B}^{0} \rightarrow a_{1}^{0} \rho^{0}$ & $0.01[0.009]$ & $\bar{B}^{0} \rightarrow b_{1}^{0} \rho^{0}$ & $0.002[-]$ \\
$B^{-} \rightarrow a_{1}^{-} \rho^{0}$ & $3.0[2.3]$ & $B^{-} \rightarrow b_{1}^{-} \rho^{0}$ & $0.36[0.27]$ \\
$B^{-} \rightarrow a_{1}^{0} \rho^{-}$ & $2.4[-]$ & $B^{-} \rightarrow b_{1}^{0} \rho^{-}$ & $0.86[-]$ \\
$\bar{B}^{0} \rightarrow a_{1}^{0} \omega$ & $0.003[0.02]$ & $\bar{B}^{0} \rightarrow b_{1}^{0} \omega$ & $0.004[0.001]$ \\
$B^{-} \rightarrow a_{1}^{-} \omega$ & $2.2[5.1]$ & $B^{-} \rightarrow b_{1}^{-} \omega$ & $0.38[0.46]$ \\
$\bar{B}^{0} \rightarrow a_{1}^{0} \phi$ & $0.0005[-]$ & $\bar{B}^{0} \rightarrow b_{1}^{0} \phi$ & $0.0002[-]$ \\
$B^{-} \rightarrow a_{1}^{-} \phi$ & $0.001[-]$ & $B^{-} \rightarrow b_{1}^{-} \phi$ & $0.0004[-]$ \\
$\bar{B}^{0} \rightarrow a_{1}^{0} K^{* 0}$ & $0.64[0.61]$ & $\bar{B}^{0} \rightarrow b_{1}^{0} K^{* 0}$ & $0.15[-]$ \\
$\bar{B}^{0} \rightarrow a_{1}^{+} K^{*-}$ & $0.92[-]$ & $\bar{B}^{0} \rightarrow b_{1}^{+} K^{*-}$ & $0.32[-]$ \\
$\bar{B}^{-} \rightarrow a_{1}^{0} K^{*-}$ & $0.86[0.81]$ & $\bar{B}^{-} \rightarrow b_{1}^{0} K^{*-}$ & $0.12[0.13]$ \\
$\bar{B}^{-} \rightarrow a_{1}^{-} \bar{K}^{* 0}$ & $0.51[-]$ & $\bar{B}^{-} \rightarrow b_{1}^{-} \bar{K}^{* 0}$ & $0.18[-]$ \\
$\bar{B}^{0} \rightarrow f_{1} \rho^{0}$ & $0.03[-]$ & $\bar{B}^{0} \rightarrow h_{1} \rho^{0}$ & $0.002[-]$ \\
$B^{-} \rightarrow f_{1} \rho^{-}$ & $4.9[-]$ & $B^{-} \rightarrow h_{1} \rho^{-}$ & $1.6[-]$ \\
$\bar{B}^{0} \rightarrow f_{1} \omega$ & $0.02[-]$ & $\bar{B}^{0} \rightarrow h_{1} \omega$ & $0.005[-]$ \\
$\bar{B}^{0} \rightarrow f_{1} \phi$ & $0.0005[-]$ & $\bar{B}^{0} \rightarrow h_{1} \phi$ & $0.0002[-]$ \\
$\bar{B}^{0} \rightarrow f_{1} K^{* 0}$ & $0.43[0.42]$ & $\bar{B}^{0} \rightarrow h_{1} K^{* 0}$ & $0.35[0.32]$ \\
$B^{-} \rightarrow f_{1} K^{*-}$ & $0.45[0.48]$ & $B^{-} \rightarrow h_{1} K^{*-}$ & $0.50[0.48]$ \\
\hline \hline
\end{tabular}

TABLE XII. Branching ratios (in units of $10^{-6}$ ) of $B \rightarrow A V$ decays, where $A$ is a strange axial-vector meson $K_{1}(1270)$ or $K_{1}(1400)$, using the ISGW2 form factors for $B \rightarrow A$ transitions and BSW [LCSR] for $B \rightarrow V$ transitions.

\begin{tabular}{lcclcc}
\hline \hline Mode & $\mathcal{B}\left(32^{\circ}\right)$ & $\mathcal{B}\left(58^{\circ}\right)$ & Mode & $\mathcal{B}\left(32^{\circ}\right)$ & $\mathcal{B}\left(58^{\circ}\right)$ \\
\hline $\bar{B}^{0} \rightarrow K_{1}^{-}(1270) \rho^{+}$ & $0.62[0.45]$ & $0.62[0.45]$ & $\bar{B}^{0} \rightarrow K_{1}^{-}(1400) \rho^{+}$ & $0.45[0.31]$ & $0.45[0.31]$ \\
$\bar{B}^{0} \rightarrow \bar{K}_{1}^{0}(1270) \rho^{0}$ & $0.001[-]$ & $0.02[-]$ & $\bar{B}^{0} \rightarrow \bar{K}_{1}^{0}(1400) \rho^{0}$ & $0.05[-]$ & $0.04[-]$ \\
$B^{-} \rightarrow K_{1}^{-}(1270) \rho^{0}$ & $0.10[0.03]$ & $0.05[0.07]$ & $B^{-} \rightarrow K_{1}^{-}(1400) \rho^{0}$ & $0.02[0.01]$ & $0.02[0.01]$ \\
$B^{-} \rightarrow \bar{K}_{1}^{0}(1270) \rho^{-}$ & $0.001[-]$ & $0.001[-]$ & $B^{-} \rightarrow \bar{K}_{1}^{0}(1400) \rho^{-}$ & $0.001[0.0006]$ & $0.001[0.0006]$ \\
$\bar{B}^{0} \rightarrow \bar{K}_{1}^{0}(1270) \omega$ & $0.0002[0.001]$ & $0.001[0.003]$ & $\bar{B}^{0} \rightarrow \bar{K}_{1}^{0}(1400) \omega$ & $0.004[0.005]$ & $0.003[0.007]$ \\
$B^{-} \rightarrow K_{1}^{-}(1270) \omega$ & $0.06[0.16]$ & $0.07[0.15]$ & $B^{-} \rightarrow K_{1}^{-}(1400) \omega$ & $0.06[0.07]$ & $0.06[0.07]$ \\
$\bar{B}^{0} \rightarrow \bar{K}_{1}^{0}(1270) \phi$ & $0.004[-]$ & $0.25[-]$ & $\bar{B}^{0} \rightarrow \bar{K}_{1}^{0}(1400) \phi$ & $0.87[-]$ & $0.66[-]$ \\
$B^{-} \rightarrow K_{1}^{-}(1270) \phi$ & $0.004[-]$ & $0.27[-]$ & $B^{-} \rightarrow K_{1}^{-}(1400) \phi$ & $0.93[-]$ & $0.69[-]$ \\
$\bar{B}^{0} \rightarrow K_{1}^{0}(1270) \bar{K}^{* 0}$ & $0.96[0.76]$ & $0.96[0.76]$ & $\bar{B}^{0} \rightarrow K_{1}^{0}(1400) \bar{K}^{* 0}$ & $0.67[0.52]$ & $0.67[0.52]$ \\
$\bar{B}^{0} \rightarrow \bar{K}_{1}^{0}(1270) K^{* 0}$ & $0.0007[-]$ & $0.31[-]$ & $\bar{B}^{0} \rightarrow \bar{K}_{1}^{0}(1400) K^{* 0}$ & $1.1[-]$ & $0.82[-]$ \\
$B^{-} \rightarrow K_{1}^{-}(1270) K^{* 0}$ & $0.0007[-]$ & $0.33[-]$ & $B^{-} \rightarrow K_{1}^{-}(1400) K^{* 0}$ & $1.2[-]$ & $0.88[-]$ \\
$B^{-} \rightarrow K_{1}^{0}(1270) K^{*-}$ & $1.0[0.82]$ & $1.0[0.82]$ & $B^{-} \rightarrow K_{1}^{0}(1400) K^{*-}$ & $0.73[0.56]$ & $0.73[0.56]$ \\
\hline \hline
\end{tabular}

\section{CONCLUSIONS}

In this work, we have presented a systematic study of exclusive charmless nonleptonic two-body $B$ decays including axial-vector mesons in the final state. Branching ratios of decays $B \rightarrow P A, B \rightarrow V A$ and $B \rightarrow A A$ (where $A, V$ and $P$ denote an axial-vector, a vector and a pseudoscalar meson, respectively) have been calculated assuming the naive factorization hypothesis and using the improved version of the nonrelativistic ISGW quark model in order to obtain form factors required for $B \rightarrow A$ transitions. Form factors for $B \rightarrow P$ and $B \rightarrow V$ transitions were obtained from the WSB model and LCSR approach. We have included contributions that arise from the effective $\Delta B=1$ weak Hamiltonian $H_{e f f}$, i.e., we have considered $W$-external and $W$-internal emissions, which have contributions of $a_{1}$ and $a_{2}$ QCD parameters, respectively, and penguin contributions given by $a_{3, \ldots, 10}$ QCD parameters. The respective factorized amplitudes of these decays are explicitly showed in appendices and their penguin contributions have been classified. We also present a comparison between $B \rightarrow A$ and $B \rightarrow V$ transitions.

We have obtained branching ratios for 141 exclusive channels $B \rightarrow A P, A V$ and $A A$ where the axial-vector meson can be a ${ }^{3} P_{1}$ or a ${ }^{1} P_{1}$ meson. We also studied the dependence of the branching ratios for $B \rightarrow K_{1} P(V, A)$ decays $\left(K_{1}=K_{1}(1270), K_{1}(1400)\right.$ are the physical strange axial-vector mesons) with respect to the mixing angle between $K_{A}$ and $K_{B}$. The best scenarios for determining this mixing angle are the decays $\bar{B}^{0} \rightarrow \bar{K}_{1}^{0}(1270) \eta^{\left({ }^{\prime}\right)}\left(K^{0}, K^{* 0}\right)$ and 
TABLE XIII. Branching ratios (in units of $10^{-6}$ ) of $B \rightarrow b_{1}\left(h_{1}\right) P$ and $B \rightarrow b_{1}\left(h_{1}\right) V$ decays, calculated with decay constants in the limit $f_{b_{1}}=f_{h_{1}}=0$.

\begin{tabular}{lc}
\hline \hline Mode & $\mathcal{B}$ \\
\hline$B^{0} \rightarrow b_{1}^{-} \pi^{+}$ & 0.0 \\
$B^{-} \rightarrow b_{1}^{-} \pi^{0}$ & 0.29 \\
$B^{-} \rightarrow b_{1}^{-} \eta$ & 0.061 \\
$B^{-} \rightarrow b_{1}^{-} \eta^{\prime}$ & 0.58 \\
$\bar{B}^{0} \rightarrow b_{1}^{-} \rho^{+}$ & 0.0 \\
$B^{-} \rightarrow b_{1}^{-} \rho^{0}$ & 0.0005 \\
$\bar{B}^{0} \rightarrow b_{1}^{0} \omega$ & 0.02 \\
$B^{-} \rightarrow b_{1}^{-} \omega$ & 0.004 \\
$\bar{B}^{0} \rightarrow h_{1} \rho^{0}$ & 0.02 \\
$\bar{B}^{0} \rightarrow h_{1} K^{* 0}$ & 0.16 \\
$B^{-} \rightarrow h_{1} K^{*-}$ & 0.34 \\
\hline \hline
\end{tabular}

TABLE XIV. Branching ratios (in units of $10^{-6}$ ) of $B \rightarrow A A$ decays, where $A$ is a nonstrange axial-vector meson, using the ISGW2 form factors.

\begin{tabular}{lc}
\hline \hline Mode & $\mathcal{B}$ \\
\hline $\bar{B}^{0} \rightarrow a_{1}^{-} a_{1}^{+}$ & 6.4 \\
$\bar{B}^{0} \rightarrow a_{1}^{0} a_{1}^{0}$ & 0.1 \\
$B^{-} \rightarrow a_{1}^{-} a_{1}^{0}$ & 3.6 \\
$\bar{B}^{0} \rightarrow a_{1}^{0} f_{1}$ & 0.02 \\
$B^{-} \rightarrow a_{1}^{-} f_{1}$ & 3.7 \\
\hline \hline
\end{tabular}

$B^{-} \rightarrow K_{1}^{-}(1270) \eta^{\left({ }^{\prime}\right)}\left(K^{0}, K^{* 0}\right)$ because their branching ratios strongly depend on the mixing angle.

Our results show that some of these decays can be reach in experiment. In fact, decays $B^{-} \rightarrow a_{1}^{0} \pi^{-}, \bar{B}^{0} \rightarrow a_{1}^{ \pm} \pi^{\mp}$, $B^{-} \rightarrow a_{1}^{-} \bar{K}^{0}, \bar{B}^{0} \rightarrow a_{1}^{+} K^{-}, \bar{B}^{0} \rightarrow f_{1} \bar{K}^{0}, B^{-} \rightarrow f_{1} K^{-}, B^{-} \rightarrow K_{1}^{-}(1400) \eta^{(')}, B^{-} \rightarrow b_{1}^{-} \bar{K}^{0}$, and $\bar{B}^{0} \rightarrow b_{1}^{+} \pi^{-}\left(K^{-}\right)$ have branching ratios of the order of $10^{-5}$.

At experimental level there is not enough information. In Ref. [5] there are only bounds for branching ratios of some charmless $B \rightarrow A P, A V, A A$ decays (see Table XVI). In general, our results are smaller that these bounds by two orders of magnitude. Our predictions $\operatorname{Br}\left(\bar{B}^{0} \rightarrow a_{1}^{-} \pi^{+}\right)=36.7 \times 10^{-6}\left[23.5 \times 10^{-6}\right]$ and $B r\left(\bar{B}^{0} \rightarrow a_{1}^{+} \pi^{-}\right)=$ $74.3 \times 10^{-6}[-]$, i.e. the $\mathrm{CP}$-averaged branching ratio $\operatorname{Br}\left(\bar{B}^{0} \rightarrow a_{1}^{\mp} \pi^{ \pm}\right)=55.5 \times 10^{-6}\left[48.9 \times 10^{-6}\right]$ is consistent with the experimental average value $\operatorname{Br}\left(\bar{B}^{0} \rightarrow a_{1}^{\mp} \pi^{ \pm}\right)=(40.9 \pm 7.6) \times 10^{-6}$ [8]. This average includes BaBar and Belle results [1].

In general, we can explain the large branching ratios for $B \rightarrow K_{1}(1400) \eta^{\left({ }^{\prime}\right)}$ as a combination of effects, the constructive interference of the terms $a_{4}$ and $a_{6}$ which are the bigger coefficients in the penguin sector of the effective Hamiltonian and the two mixing $K_{1 A}-K_{1 B}$ and $\eta-\eta^{\prime}$ involved in the decays.

Finally, we want to mention that our predictions are at the same order that ones obtained by Laporta-NardulliPham (see Tables V and VI in Ref. [8]), although our values are in general bigger, except in a few modes. On the other hand, predictions obtained in Ref. 9] (see Tables II and III) for $B \rightarrow K_{1}(1270) \phi$ and $B \rightarrow K_{1}(1400) \phi$ and our predictions for these modes (see Table XI) do not agree, except for the case with $N_{c}^{\text {eff }}=\infty$ and $\theta=58^{\circ}$ (with $\mu=2.5 \mathrm{GeV}$ or $\mu=4.4 \mathrm{GeV})$. In this case the respective branching ratios are $\mathcal{O}\left(10^{-7}\right)$.

\section{Acknowledgments}

We thank C. Ramírez from Universidad Industrial de Santander, Colombia for useful conversatios and G. López Castro from CINVESTAV, México for reading the manuscript and his valuable suggestions. The authors acknowledge financial support from Conacyt (G. C.) and Comité Central de Investigaciones of University of Tolima (J. H. M. and C. E. V.). 
TABLE XV. Branching ratios (in units of $10^{-6}$ ) of $B \rightarrow K_{1} A$ decays, where $A$ is a nonstrange axial-vector meson, using the ISGW2 form factors. The $K_{1}$ axial mesons are $K_{1}(1270)$ and $K_{1}(1400)$.

\begin{tabular}{lccccc}
\hline \hline Mode & $\mathcal{B}\left(32^{\circ}\right)$ & $\mathcal{B}\left(58^{\circ}\right)$ & Mode & \multicolumn{1}{c}{$\mathcal{B}\left(32^{\circ}\right)$} & $\mathcal{B}\left(58^{\circ}\right)$ \\
\hline $\bar{B}^{0} \rightarrow K_{1}^{-}(1270) a_{1}^{+}$ & 0.79 & 0.79 & $\bar{B}^{0} \rightarrow K_{1}^{-}(1400) a_{1}^{+}$ & 0.49 & 0.49 \\
$\bar{B}^{0} \rightarrow \bar{K}_{1}^{0}(1270) a_{1}^{0}$ & 0.002 & 0.03 & $\bar{B}^{0} \rightarrow \bar{K}_{1}^{0}(1400) a_{1}^{0}$ & 0.08 & 0.06 \\
$B^{-} \rightarrow K_{1}^{-}(1270) a_{1}^{0}$ & 0.12 & 0.06 & $B^{-} \rightarrow K_{1}^{-}(1400) a_{1}^{0}$ & 0.03 & 0.03 \\
$B^{-} \rightarrow \bar{K}_{1}^{0}(1270) a_{1}^{-}$ & 0.002 & 0.002 & $B^{-} \rightarrow \bar{K}_{1}^{0}(1400) a_{1}^{-}$ & 0.001 & 0.001 \\
$\bar{B}^{0} \rightarrow \bar{K}_{1}^{0}(1270) f_{1}$ & 0.44 & 0.53 & $\bar{B}^{0} \rightarrow \bar{K}_{1}^{0}(1400) f_{1}$ & 0.48 & 0.44 \\
$B^{-} \rightarrow K_{1}^{-}(1270) f_{1}$ & 0.15 & 0.27 & $B^{-} \rightarrow K_{1}^{-}(1400) f_{1}$ & 0.34 & 0.29 \\
\hline \hline
\end{tabular}

TABLE XVI. Experimental bounds for branching ratios of some charmless $B \rightarrow A P, A V$ and $A A$ decays reported in $[5]$.

\begin{tabular}{lc}
\hline \hline Mode & $\mathcal{B}^{\text {exp }}$ \\
\hline$B^{0} \rightarrow a_{1}^{+}(1260) \pi^{ \pm}$ & $<4.9 \times 10^{-4}$ \\
$B^{0} \rightarrow a_{1}^{0}(1260) \pi^{0}$ & $<1.1 \times 10^{-3}$ \\
$B^{0} \rightarrow a_{1}^{+}(1260) \rho^{-}$ & $<3.4 \times 10^{-3}$ \\
$B^{0} \rightarrow a_{1}^{0}(1260) \rho^{0}$ & $<2.4 \times 10^{-3}$ \\
$B^{0} \rightarrow K_{1}^{+}(1400) \pi^{-}$ & $<1.1 \times 10^{-3}$ \\
$B^{0} \rightarrow a_{1}^{+}(1260) K^{-}$ & $<2.3 \times 10^{-4}$ \\
$B^{0} \rightarrow K_{1}^{0}(1400) \rho^{0}$ & $<3.0 \times 10^{-3}$ \\
$B^{0} \rightarrow K_{1}^{0}(1400) \phi$ & $<5.0 \times 10^{-3}$ \\
$B^{+} \rightarrow a_{1}^{+}(1260) \pi^{0}$ & $<1.7 \times 10^{-3}$ \\
$B^{+} \rightarrow a_{1}^{0}(1260) \pi^{+}$ & $<9.0 \times 10^{-4}$ \\
$B^{+} \rightarrow a_{1}^{+}(1260) \rho^{0}$ & $<6.2 \times 10^{-4}$ \\
$B^{+} \rightarrow a_{1}^{+}(1260) a_{1}^{0}(1260)$ & $<1.3 \%$ \\
$B^{+} \rightarrow K_{1}^{0}(1400) \pi^{+}$ & $<2.6 \times 10^{-3}$ \\
$B^{+} \rightarrow K_{1}^{+}(1400) \rho^{0}$ & $<7.8 \times 10^{-4}$ \\
$B^{+} \rightarrow K_{1}^{+}(1400) \phi$ & $<1.1 \times 10^{-3}$ \\
\hline \hline
\end{tabular}

\section{APPENDIX A: MATRIX ELEMENTS FOR $B$ DECAYS TO AN AXIAL AND A PSEUDOSCALAR MESON}

$$
\begin{gathered}
\mathcal{M}\left(\bar{B}^{0} \rightarrow a_{1}^{-} \pi^{+}\right)=2 m_{a_{1}} f_{a_{1}} F_{1}^{B \rightarrow \pi}\left(m_{a_{1}}^{2}\right)\left\{V_{u b} V_{u d}^{*} a_{1}-V_{t b} V_{t d}^{*}\left(a_{4}+a_{10}\right)\right\} \\
\mathcal{M}\left(\bar{B}^{0} \rightarrow a_{1}^{+} \pi^{-}\right)=2 i m_{\pi} f_{\pi} V_{0}^{B \rightarrow a_{1}}\left(m_{\pi}^{2}\right)\left\{V_{u b} V_{u d}^{*} a_{1}\right. \\
\left.\quad-V_{t b} V_{t d}^{*}\left[a_{4}+a_{10}+2\left(a_{6}+a_{8}\right) \frac{m_{\pi}^{2}}{\left(m_{u}+m_{d}\right)\left(m_{b}-m_{u}\right)}\right]\right\} \\
\mathcal{M}\left(\bar{B}^{0} \rightarrow a_{1}^{0} \pi^{0}\right)= \\
2 i m_{\pi} f_{\pi} V_{0}^{B \rightarrow a_{1}}\left(m_{\pi}^{2}\right)\left\{V_{u b} V_{u d}^{*} a_{2}\right. \\
\left.\quad-V_{t b} V_{t d}^{*}\left[-a_{4}-\left(2 a_{6}-a_{8}\right) \frac{m_{\pi}^{2}}{2 m_{d}\left(m_{b}-m_{d}\right)}-\frac{1}{2}\left(3 a_{7}-3 a_{9}-a_{10}\right)\right]\right\} \\
+2 m_{a_{1}} f_{a_{1}} F_{1}^{B \rightarrow \pi}\left(m_{a_{1}}^{2}\right)\left\{V_{u b} V_{u d}^{*} a_{2}-V_{u b} V_{u d}^{*}\left[-a_{4}-\frac{1}{2}\left(3 a_{7}-3 a_{9}-a_{10}\right)\right]\right\}
\end{gathered}
$$




$$
\begin{aligned}
& \mathcal{M}\left(B^{-} \rightarrow a_{1}^{-} \pi^{0}\right)=2 i m_{\pi} f_{\pi} V_{0}^{B \rightarrow a_{1}}\left(m_{\pi}^{2}\right)\left\{V_{u b} V_{u d}^{*} a_{2}\right. \\
& \left.-V_{t b} V_{t d}^{*}\left[-a_{4}-\frac{1}{2}\left(3 a_{7}-3 a_{9}-a_{10}\right)-\left(2 a_{6}-a_{8}\right) \frac{m_{\pi}^{2}}{2 m_{d}\left(m_{b}-m_{d}\right)}\right]\right\} \\
& +2 m_{a_{1}} f_{a_{1}} F_{1}^{B \rightarrow \pi}\left(m_{a_{1}}^{2}\right)\left\{V_{u b} V_{u d}^{*} a_{1}-V_{t b} V_{t d}^{*}\left(a_{4}+a_{10}\right)\right\} \\
& \mathcal{M}\left(B^{-} \rightarrow a_{1}^{0} \pi^{-}\right)=2 i m_{\pi} f_{\pi} V_{0}^{B \rightarrow a_{1}}\left(m_{\pi}^{2}\right)\left\{V_{u b} V_{u d}^{*} a_{1}-V_{t b} V_{t d}^{*}\left[a_{4}+a_{10}+2\left(a_{6}+a_{8}\right) \frac{m_{\pi^{-}}^{2}}{\left(m_{d}+m_{u}\right)\left(m_{b}-m_{u}\right)}\right]\right\} \\
& +2 m_{a_{1}} f_{a_{1}} F_{1}^{B \rightarrow \pi}\left(m_{a_{1}}^{2}\right)\left\{V_{u b} V_{u d}^{*} a_{2}-V_{t b} V_{t d}^{*}\left[-a_{4}-\frac{1}{2}\left(3 a_{7}-3 a_{9}-a_{10}\right)\right]\right\} \\
& \mathcal{M}\left(\bar{B}^{0} \rightarrow a_{1}^{0} \eta^{\left({ }^{\prime}\right)}\right)=2 i m_{\eta^{(\prime)}} f_{\eta^{\left({ }^{\prime}\right)}}^{u} V_{0}^{B \rightarrow a_{1}}\left(m_{\eta^{(\prime)}}^{2}\right)\left\{V_{u b} V_{u d}^{*} a_{2}\right. \\
& \left.-V_{t b} V_{t d}^{*}\left[2 a_{3}+a_{4}-2 a_{5}+\left(2 a_{6}-a_{8}\right) \frac{m_{\eta^{(\prime)}}^{2}}{2 m_{s}\left(m_{b}-m_{d}\right)}\left(\frac{f_{\eta^{(\prime)}}^{s}}{f_{\eta^{\left({ }^{\prime}\right)}}^{u}}-1\right) r_{\eta^{(\prime)}}-\frac{1}{2}\left(a_{7}-a_{9}+a_{10}\right)\right]\right\} \\
& +2 m_{a_{1}} f_{a_{1}} F_{1}^{B \rightarrow \eta^{\left({ }^{\prime}\right)}}\left(m_{a_{1}}^{2}\right)\left\{V_{u b} V_{u d}^{*} a_{2}-V_{t b} V_{t d}^{*}\left[-a_{4}+\frac{1}{2} a_{10}-\frac{3}{2}\left(a_{7}-a_{9}\right)\right]\right\} \\
& -2 i m_{\eta^{\left({ }^{\prime}\right)}} f_{\eta^{\left({ }^{\prime}\right)}}^{s} V_{0}^{B \rightarrow a_{1}}\left(m_{\eta^{\left({ }^{\prime}\right)}}^{2}\right)\left\{V_{t b} V_{t d}^{*}\left[a_{3}-a_{5}+\frac{1}{2}\left(a_{7}-a_{9}\right)\right]\right\} \\
& +2 i m_{\eta^{\left({ }^{\prime}\right)}} f_{\eta^{\left({ }^{\prime}\right)}}^{c} V_{0}^{B \rightarrow a_{1}}\left(m_{\eta^{\left({ }^{\prime}\right)}}^{2}\right)\left\{V_{c b} V_{c d}^{*} a_{2}-V_{t b} V_{t d}^{*}\left(a_{3}-a_{5}-a_{7}+a_{9}\right)\right\} \\
& \mathcal{M}\left(B^{-} \rightarrow a_{1}^{-} \eta^{\left({ }^{\prime}\right)}\right)=2 i m_{\eta^{(\prime)}} f_{\eta^{\left({ }^{\prime}\right)}}^{u} V_{0}^{B \rightarrow a_{1}}\left(m_{\eta^{\left({ }^{\prime}\right)}}^{2}\right)\left\{V_{u b} V_{u d}^{*} a_{2}\right. \\
& \left.-V_{t b} V_{t d}^{*}\left[2 a_{3}+a_{4}-2 a_{5}-\frac{1}{2}\left(a_{7}-a_{9}+a_{10}\right)+\left(2 a_{6}-a_{8}\right) \frac{m_{\eta^{(\prime)}}^{2}}{2 m_{s}\left(m_{b}-m_{d}\right)}\left(\frac{f_{\eta^{(\prime)}}^{s}}{f_{\eta^{\left({ }^{\prime}\right)}}^{u}}-1\right) r_{\eta^{\left({ }^{\prime}\right)}}\right]\right\} \\
& +2 m_{a_{1}} f_{a_{1}} F_{1}^{B \rightarrow \eta^{\left({ }^{\prime}\right)}}\left(m_{a_{1}}^{2}\right)\left\{V_{u b} V_{u d}^{*} a_{1}-V_{t b} V_{t d}^{*}\left(a_{4}+a_{10}\right)\right\} \\
& -2 i m_{\eta^{\left({ }^{\prime}\right)}} f_{\eta^{\left({ }^{\prime}\right)}}^{s} V_{0}^{B \rightarrow a_{1}}\left(m_{\eta^{\left(^{\prime}\right)}}^{2}\right)\left\{V_{t b} V_{t d}^{*}\left[2 a_{3}+a_{4}-2 a_{5}-\frac{1}{2}\left(a_{7}-a_{9}\right)\right]\right\} \\
& +2 i m_{\eta^{\left({ }^{\prime}\right)}} f_{\eta^{\left({ }^{\prime}\right)}}^{c} V_{0}^{B \rightarrow a_{1}}\left(m_{\eta^{\left({ }^{\prime}\right)}}^{2}\right)\left\{V_{c b} V_{c d}^{*} a_{2}-V_{t b} V_{t d}^{*}\left(a_{3}-a_{5}-a_{7}+a_{9}\right)\right\} \\
& \mathcal{M}\left(\bar{B}^{0} \rightarrow a_{1}^{0} \bar{K}^{0}\right)=2 m_{a_{1}} f_{a_{1}} F_{1}^{B \rightarrow K}\left(m_{a_{1}}^{2}\right)\left\{V_{u b} V_{u s}^{*} a_{2}-V_{t b} V_{t s}^{*} \frac{3}{2}\left(-a_{7}+a_{9}\right)\right\} \\
& -2 i m_{K} f_{K} V_{0}^{B \rightarrow a_{1}}\left(m_{K}^{2}\right) V_{t b} V_{t s}^{*}\left[a_{4}-\frac{1}{2} a_{10}+\left(2 a_{6}-a_{8}\right) \frac{m_{K}^{2}}{\left(m_{s}+m_{d}\right)\left(m_{b}-m_{d}\right)}\right]
\end{aligned}
$$




$$
\begin{aligned}
& \mathcal{M}\left(\bar{B}^{0} \rightarrow a_{1}^{+} K^{-}\right)=2 i m_{K} f_{K} V_{0}^{B \rightarrow a_{1}}\left(m_{K}^{2}\right)\left\{V_{u b} V_{u s}^{*} a_{1}\right. \\
& \left.-V_{t b} V_{t s}^{*}\left[a_{4}+a_{10}+2\left(a_{6}+a_{8}\right) \frac{m_{K}^{2}}{\left(m_{s}+m_{u}\right)\left(m_{b}-m_{u}\right)}\right]\right\} \\
& \mathcal{M}\left(B^{-} \rightarrow a_{1}^{0} K^{-}\right)=2 i m_{K} f_{K} V_{0}^{B \rightarrow a_{1}}\left(m_{K}^{2}\right)\left\{V_{u b} V_{u s}^{*} a_{1}-V_{t b} V_{t s}^{*}\left[a_{4}+a_{10}+2\left(a_{6}+a_{8}\right) \frac{m_{K^{-}}^{2}}{\left(m_{s}+m_{u}\right)\left(m_{b}-m_{u}\right)}\right]\right\} \\
& +2 m_{a_{1}} f_{a_{1}} F_{1}^{B \rightarrow K}\left(m_{a_{1}}^{2}\right)\left(\epsilon^{*} \cdot p_{B}\right)\left\{V_{u b} V_{u s}^{*} a_{2}-V_{t b} V_{t s}^{*} \frac{3}{2}\left(-a_{7}+a_{9}\right)\right\} \\
& \mathcal{M}\left(B^{-} \rightarrow a_{1}^{-} \bar{K}^{0}\right)=-2 i m_{K} f_{K} V_{0}^{B \rightarrow a_{1}}\left(m_{K}^{2}\right) V_{t b} V_{t s}^{*}\left[a_{4}-\frac{1}{2} a_{10}+\left(2 a_{6}-a_{8}\right) \frac{m_{K}^{2}}{\left(m_{s}+m_{d}\right)\left(m_{b}-m_{d}\right)}\right] \\
& \mathcal{M}\left(\bar{B}^{0} \rightarrow f_{1} \pi^{0}\right)=2 i m_{\pi} f_{\pi} V_{0}^{B \rightarrow f_{1}}\left(m_{\pi}^{2}\right)\left\{V_{u b} V_{u d}^{*} a_{2}-V_{t b} V_{t d}^{*}\left[a_{4}-\frac{1}{2}\left(3 a_{7}-3 a_{9}+a_{10}\right)+\left(2 a_{6}-a_{8}\right) \frac{m_{\pi^{0}}^{2}}{2 m_{d}\left(m_{b}-m_{d}\right)}\right]\right\} \\
& +2 m_{f_{1}} f_{f_{1}} F_{1}^{B \rightarrow \pi}\left(m_{f_{1}}^{2}\right)\left\{V_{u b} V_{u d}^{*} a_{2}-V_{t b} V_{t d}^{*}\left[2 a_{3}+a_{4}-2 a_{5}-\frac{1}{2}\left(a_{7}-a_{9}+a_{10}\right)\right]\right\} \\
& \mathcal{M}\left(B^{-} \rightarrow f_{1} \pi^{-}\right)=2 i m_{\pi} f_{\pi} V_{0}^{B \rightarrow f_{1}}\left(m_{\pi}^{2}\right)\left\{V_{u b} V_{u d}^{*} a_{1}-V_{t b} V_{t d}^{*}\left[a_{4}+a_{10}+2\left(a_{6}+a_{8}\right) \frac{m_{\pi^{-}}^{2}}{\left(m_{d}+m_{u}\right)\left(m_{b}-m_{u}\right)}\right]\right\} \\
& +2 m_{f_{1}} f_{f_{1}} F_{1}^{B \rightarrow \pi}\left(m_{f_{1}}^{2}\right)\left\{V_{u b} V_{u d}^{*} a_{2}-V_{t b} V_{t d}^{*}\left[2 a_{3}+a_{4}-2 a_{5}-\frac{1}{2}\left(a_{7}-a_{9}+a_{10}\right)\right]\right\} \\
& \mathcal{M}\left(\bar{B}^{0} \rightarrow f_{1} \eta^{\left({ }^{\prime}\right)}\right)=2 i m_{\eta^{\left({ }^{\prime}\right)}} f_{\eta^{\left({ }^{\prime}\right)}}^{u} V_{0}^{B \rightarrow f_{1}}\left(m_{\eta^{\left({ }^{\prime}\right)}}^{2}\right)\left\{V_{u b} V_{u d}^{*} a_{2}\right. \\
& \left.-V_{t b} V_{t d}^{*}\left[2 a_{3}+a_{4}-2 a_{5}-\frac{1}{2}\left(a_{7}-a_{9}+a_{10}\right)+\left(2 a_{6}-a_{8}\right) \frac{m_{\eta^{(\prime)}}^{2}}{2 m_{d}\left(m_{b}-m_{d}\right)}\left(\frac{f_{\left.\eta^{(}\right)}^{s}}{f_{\eta^{\left({ }^{\prime}\right.}}^{u}}-1\right) r_{\eta^{\left({ }^{\prime}\right)}}\right]\right\} \\
& +2 m_{f_{1}} f_{f_{1}} F_{1}^{B \rightarrow \eta^{\left({ }^{\prime}\right)}}\left(m_{f_{1}}^{2}\right)\left\{V_{u b} V_{u d}^{*} a_{2}-V_{t b} V_{t d}^{*}\left[2 a_{3}+a_{4}-2 a_{5}-\frac{1}{2}\left(a_{7}-a_{9}+a_{10}\right)\right]\right\} \\
& -2 i m_{\eta^{(\prime)}} f_{\eta^{\left({ }^{\prime}\right)}}^{s} V_{0}^{B \rightarrow f_{1}}\left(m_{\eta^{\left(^{\prime}\right)}}^{2}\right)\left\{V_{t b} V_{t d}^{*}\left[a_{3}-a_{5}+\frac{1}{2}\left(a_{7}-a_{9}\right)\right]\right\} \\
& +2 i m_{\eta^{\left({ }^{\prime}\right)}} f_{\eta^{\left({ }^{\prime}\right)}}^{c} V_{0}^{B \rightarrow f_{1}}\left(m_{\eta^{\left({ }^{\prime}\right)}}^{2}\right)\left\{V_{c b} V_{c d}^{*} a_{2}-V_{t b} V_{t d}^{*}\left[a_{3}-a_{5}-a_{7}+a_{9}\right]\right\} \\
& \mathcal{M}\left(\bar{B}^{0} \rightarrow f_{1} \bar{K}^{0}\right)=2 m_{f_{1}} f_{f_{1}} F_{1}^{B \rightarrow K}\left(m_{f_{1}}^{2}\right)\left\{V_{u b} V_{u s}^{*} a_{2}-V_{t b} V_{t s}^{*}\left[2 a_{3}-2 a_{5}-\frac{1}{2}\left(a_{7}-a_{9}\right)\right]\right\} \\
& -2 i m_{K} f_{K} V_{0}^{B \rightarrow f_{1}}\left(m_{K}^{2}\right) V_{t b} V_{t s}^{*}\left[a_{4}-\frac{1}{2} a_{10}+\left(2 a_{6}-a_{8}\right) \frac{m_{K}^{2}}{\left(m_{s}+m_{d}\right)\left(m_{b}-m_{d}\right)}\right]
\end{aligned}
$$




$$
\begin{aligned}
& \mathcal{M}\left(B^{-} \rightarrow f_{1} K^{-}\right)=2 i m_{K} f_{K} V_{0}^{B \rightarrow f_{1}}\left(m_{K}^{2}\right)\left\{V_{u b} V_{u s}^{*} a_{1}-V_{t b} V_{t s}^{*}\left[a_{4}+a_{10}+2\left(a_{6}+a_{8}\right) \frac{m_{K}^{2}}{\left(m_{s}+m_{u}\right)\left(m_{b}-m_{u}\right)}\right]\right\} \\
& +2 m_{f_{1}} f_{f_{1}} F_{1}^{B \rightarrow K}\left(m_{f_{1}}^{2}\right)\left\{V_{u b} V_{u s}^{*} a_{2}-V_{t b} V_{t s}^{*}\left[2 a_{3}-2 a_{5}-\frac{1}{2}\left(a_{7}-a_{9}\right)\right]\right\} \\
& \mathcal{M}\left(\bar{B}^{0} \rightarrow K_{1}^{-} \pi^{+}\right)=2 i m_{\pi} f_{\pi} V_{0}^{B \rightarrow K_{1}}\left(m_{\pi}^{2}\right)\left\{V_{u b} V_{u s}^{*} a_{1}-V_{t b} V_{t s}^{*}\left(a_{4}+a_{10}\right)\right\} \\
& \mathcal{M}\left(\bar{B}^{0} \rightarrow \bar{K}_{1}^{0} \pi^{0}\right)=2 i m_{\pi} f_{\pi} V_{0}^{B \rightarrow K_{1}^{0}}\left(m_{\pi}^{2}\right)\left\{V_{u b} V_{u s}^{*} a_{2}-V_{t b} V_{t s}^{*} \frac{3}{2}\left(-a_{7}+a_{9}\right)\right\} \\
& -2 m_{K_{1}} f_{K_{1}} F_{1}^{B \rightarrow \pi}\left(m_{K_{1}}^{2}\right) V_{t b} V_{t s}^{*}\left[a_{4}-\frac{1}{2} a_{10}\right] \\
& \mathcal{M}\left(B^{-} \rightarrow K_{1}^{-} \pi^{0}\right)=2 i m_{\pi} f_{\pi} V_{0}^{B \rightarrow K_{1}}\left(m_{\pi}^{2}\right)\left\{V_{u b} V_{u s}^{*} a_{2}-V_{t b} V_{t s}^{*} \frac{3}{2}\left(-a_{7}+a_{9}\right)\right\} \\
& +2 m_{K_{1}} f_{K_{1}} F_{1}^{B \rightarrow \pi}\left(m_{K_{1}(1270)}^{2}\right)\left\{V_{u b} V_{u s}^{*} a_{1}-V_{t b} V_{t s}^{*}\left(a_{4}+a_{10}\right)\right\} \\
& \mathcal{M}\left(B^{-} \rightarrow \bar{K}_{1}^{0} \pi^{-}\right)=-2 m_{K_{1}} f_{K_{1}} F_{1}^{B \rightarrow \pi}\left(m_{K_{1}}^{2}\right) V_{t b} V_{t s}^{*}\left\{\left(a_{4}-\frac{1}{2} a_{10}\right)\right\}
\end{aligned}
$$

$$
\begin{aligned}
\mathcal{M}\left(\bar{B}^{0} \rightarrow \bar{K}_{1}^{0} \eta^{\left({ }^{\prime}\right)}\right)= & 2 i m_{\eta^{\left({ }^{\prime}\right)}} f_{\eta^{\left({ }^{\prime}\right.}}^{u} V_{0}^{B \rightarrow K_{1}}\left(m_{\eta^{\left({ }^{\prime}\right)}}^{2}\right)\left\{V_{u b} V_{u s}^{*} a_{2}-V_{t b} V_{t s}^{*}\left[2\left(a_{3}-a_{5}\right)-\frac{1}{2}\left(a_{7}-a_{9}\right)\right]\right\} \\
& -2 m_{K_{1}} f_{K_{1}^{0}} F_{1}^{B \rightarrow \eta^{\left({ }^{\prime}\right)}}\left(m_{K_{1}^{0}}^{2}\right)\left\{V_{t b} V_{t s}^{*}\left(a_{4}-\frac{1}{2} a_{10}\right)\right\} \\
& -2 i m_{\eta^{\left({ }^{\prime}\right)}} f_{\eta^{\left({ }^{\prime}\right)}}^{s} V_{0}^{B \rightarrow K_{1}}\left(m_{\eta^{\left({ }^{\prime}\right)}}^{2}\right)\left\{V_{t b} V_{t s}^{*}\right. \\
& {\left.\left[a_{3}+a_{4}-a_{5}+\frac{1}{2}\left(a_{7}-a_{9}-a_{10}\right)+\left(2 a_{6}-a_{8}\right) \frac{m_{\eta^{\left({ }^{\prime}\right)}}^{2}}{2 m_{s}\left(m_{b}-m_{s}\right)}\left(1-\frac{f_{\eta^{\left({ }^{\prime}\right)}}^{u}}{f_{\eta^{\left({ }^{\prime}\right)}}^{s}}\right)\right]\right\} } \\
& +2 i m_{\eta^{\left({ }^{\prime}\right)}} f_{\eta^{\left({ }^{\prime}\right)}}^{c} V_{0}^{B \rightarrow K_{1}}\left(m_{\eta^{\left({ }^{\prime}\right)}}^{2}\right)\left\{V_{u b} V_{u s}^{*} a_{2}-V_{t b} V_{t s}^{*}\left(a_{3}-a_{5}-a_{7}+a_{9}\right)\right\}
\end{aligned}
$$$$
\mathcal{M}\left(B^{-} \rightarrow K_{1}^{-} \eta^{\left({ }^{\prime}\right)}\right)=2 i m_{\eta^{\left({ }^{\prime}\right)}} f_{\eta^{\left({ }^{\prime}\right)}}^{u} V_{0}^{B \rightarrow K_{1}}\left(m_{\eta^{(\prime)}}^{2}\right)\left\{V_{u b} V_{u s}^{*} a_{2}-V_{t b} V_{t s}^{*}\left[2 a_{3}-2 a_{5}-\frac{1}{2}\left(a_{7}-a_{9}\right)\right]\right\}
$$$$
+2 m_{K_{1}} f_{K_{1}} F_{1}^{B \rightarrow \eta^{(\prime)}}\left(m_{K_{1}}^{2}\right)\left\{V_{u b} V_{u s}^{*} a_{1}-V_{t b} V_{t s}^{*}\left(a_{4}+a_{10}\right)\right\}
$$

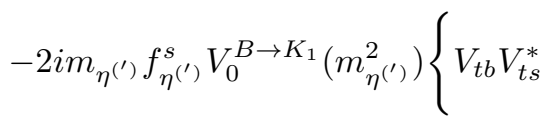$$
\left.\left[a_{3}+a_{4}-a_{5}+\frac{1}{2}\left(a_{7}-a_{9}-a_{10}\right)+\left(2 a_{6}-a_{8}\right) \frac{m_{\eta^{\left({ }^{\prime}\right)}}^{2}}{2 m_{s}\left(m_{b}-m_{s}\right)}\left(1-\frac{f_{\eta^{\left({ }^{\prime}\right)}}^{u}}{f_{\eta^{\left({ }^{\prime}\right)}}^{s}}\right)\right]\right\}
$$$$
+2 i m_{\eta^{\left({ }^{\prime}\right)}} f_{\eta^{\left({ }^{\prime}\right)}}^{c} V_{0}^{B \rightarrow K_{1}}\left(m_{\eta^{\left({ }^{\prime}\right)}}^{2}\right)\left\{V_{c b} V_{c s}^{*} a_{2}-V_{t b} V_{t s}^{*}\left(a_{3}-a_{5}-a_{7}+a_{9}\right)\right\}
$$ 


$$
\begin{gathered}
\mathcal{M}\left(\bar{B}^{0} \rightarrow K_{1}^{0} \bar{K}^{0}\right)=-2 m_{K_{1}} f_{K_{1}} F_{1}^{B \rightarrow K}\left(m_{K_{1}}^{2}\right) V_{t b} V_{t d}^{*}\left\{\left(a_{4}-\frac{1}{2} a_{10}\right)\right\} \\
\mathcal{M}\left(\bar{B}^{0} \rightarrow \bar{K}_{1}^{0} K^{0}\right)=-2 i m_{K} f_{K} V_{0}^{B \rightarrow K_{1}}\left(m_{K}^{2}\right) V_{t b} V_{t d}^{*}\left\{\left[a_{4}-\frac{1}{2} a_{10}+\left(2 a_{6}-a_{8}\right) \frac{m_{K}^{2}}{\left(m_{s}+m_{d}\right)\left(m_{b}-m_{s}\right)}\right]\right\} \\
\mathcal{M}\left(B^{-} \rightarrow K_{1}^{-} K^{0}\right)=-2 i m_{K} f_{K} V_{0}^{B \rightarrow K_{1}}\left(m_{K}^{2}\right) V_{t b} V_{t d}^{*}\left\{\left[a_{4}-\frac{1}{2} a_{10}+\left(2 a_{6}-a_{8}\right) \frac{m_{K^{0}}^{2}}{\left(m_{s}+m_{d}\right)\left(m_{b}-m_{s}\right)}\right]\right\} \\
\mathcal{M}\left(B^{-} \rightarrow K_{1}^{0} K^{-}\right)=-2 m_{K_{1}} f_{K_{1}} F_{1}^{B \rightarrow K}\left(m_{K_{1}}^{2}\right) V_{t b} V_{t d}^{*}\left\{\left(a_{4}-\frac{1}{2} a_{10}\right)\right\}
\end{gathered}
$$

\section{APPENDIX B: MATRIX ELEMENTS FOR $B$ DECAYS TO AN AXIAL AND A VECTOR MESON}

$$
\begin{aligned}
& \mathcal{M}\left(\bar{B}^{0} \rightarrow a_{1}^{-} \rho^{+}\right)=m_{a_{1}} f_{a_{1}}\left\{V_{u b} V_{u d}^{*} a_{1}-V_{t b} V_{t d}^{*}\left(a_{4}+a_{10}\right)\right\}\left(\frac{2 V^{B \rightarrow \rho}\left(m_{a_{1}}^{2}\right)}{\left(m_{B}+m_{\rho}\right)} \epsilon_{\mu \nu \alpha \beta} \epsilon_{a_{1}}^{\mu} \epsilon_{\rho}^{\nu} p_{B}^{\alpha} p_{\rho}^{\beta}\right. \\
& \left.-i\left(m_{B}+m_{\rho}\right) A_{1}^{B \rightarrow \rho}\left(m_{a_{1}}^{2}\right)\left(\epsilon_{\rho} \cdot \epsilon_{a_{1}}\right)+\frac{i A_{2}^{B \rightarrow \rho}\left(m_{a_{1}}^{2}\right)}{\left(m_{B}+m_{\rho}\right)}\left(\epsilon_{\rho} \cdot p_{B}\right)\left(\epsilon_{a_{1}} \cdot p_{B}\right)\right) \\
& \mathcal{M}\left(\bar{B}^{0} \rightarrow a_{1}^{+} \rho^{-}\right)=-m_{\rho} f_{\rho}\left\{V_{u b} V_{u d}^{*} a_{1}-V_{t b} V_{t d}^{*}\left(a_{4}+a_{10}\right)\right\}\left(\frac{2 A^{B \rightarrow a_{1}}\left(m_{\rho}^{2}\right)}{\left(m_{B}+m_{a_{1}}\right)} \epsilon_{\mu \nu \alpha \beta} \epsilon_{\rho}^{\mu} \epsilon_{a_{1}}^{\nu} p_{B}^{\alpha} p_{a_{1}}^{\beta}\right. \\
& \left.-i\left(m_{B}+m_{a_{1}}\right) V_{1}^{B \rightarrow a_{1}}\left(m_{\rho}^{2}\right)\left(\epsilon_{a_{1}} \cdot \epsilon_{\rho}\right)+\frac{i V_{2}^{B \rightarrow a_{1}}\left(m_{\rho}^{2}\right)}{\left(m_{B}+m_{a_{1}}\right)}\left(\epsilon_{a_{1}} \cdot p_{B}\right)\left(\epsilon_{\rho} \cdot p_{B}\right)\right) \\
& \mathcal{M}\left(\bar{B}^{0} \rightarrow a_{1}^{0} \rho^{0}\right)=-m_{\rho} f_{\rho}\left\{V_{u b} V_{u d}^{*} a_{2}-V_{t b} V_{t d}^{*}\left[-a_{4}+\frac{1}{2}\left(3 a_{7}+3 a_{9}+a_{10}\right)\right]\right\}\left(\frac{2 A^{B \rightarrow a_{1}}\left(m_{\rho}^{2}\right)}{\left(m_{B}+m_{a_{1}}\right)} \epsilon_{\mu \nu \alpha \beta} \epsilon_{\rho}^{\mu} \epsilon_{a_{1}}^{\nu} p_{B}^{\alpha} p_{a_{1}}^{\beta}\right. \\
& \left.-i\left(m_{B}+m_{a_{1}}\right) V_{1}^{B \rightarrow a_{1}}\left(m_{\rho}^{2}\right)\left(\epsilon_{a_{1}} \cdot \epsilon_{\rho}\right)+\frac{i V_{2}^{B \rightarrow a_{1}}\left(m_{\rho}^{2}\right)}{\left(m_{B}+m_{a_{1}}\right)}\left(\epsilon_{a_{1}} \cdot p_{B}\right)\left(\epsilon_{\rho} \cdot p_{B}\right)\right) \\
& +m_{a_{1}} f_{a_{1}}\left\{V_{u b} V_{u d}^{*} a_{2}-V_{t b} V_{t d}^{*}\left[-a_{4}-\frac{1}{2}\left(3 a_{7}-3 a_{9}-a_{10}\right)\right]\right\}\left(\frac{2 V^{B \rightarrow \rho}\left(m_{a_{1}}^{2}\right)}{\left(m_{B}+m_{\rho}\right)} \epsilon_{\mu \nu \alpha \beta} \epsilon_{a_{1}}^{\mu} \epsilon_{\rho}^{\nu} p_{B}^{\alpha} p_{\rho}^{\beta}\right. \\
& \left.-i\left(m_{B}+m_{\rho}\right) A_{1}^{B \rightarrow \rho}\left(m_{a_{1}}^{2}\right)\left(\epsilon_{\rho} \cdot \epsilon_{a_{1}}\right)+\frac{i A_{2}^{B \rightarrow \rho}\left(m_{a_{1}}^{2}\right)}{\left(m_{B}+m_{\rho}\right)}\left(\epsilon_{\rho} \cdot p_{B}\right)\left(\epsilon_{a_{1}} \cdot p_{B}\right)\right) \\
& \mathcal{M}\left(B^{-} \rightarrow a_{1}^{-} \rho^{0}\right)=-m_{\rho} f_{\rho}\left\{V_{u b} V_{u d}^{*} a_{2}\right\}\left(\frac{2 A^{B \rightarrow a_{1}}\left(m_{\rho}^{2}\right)}{\left(m_{B}+m_{a_{1}}\right)} \epsilon_{\mu \nu \alpha \beta} \epsilon_{\rho}^{\mu} \epsilon_{a_{1}}^{\nu} p_{B}^{\alpha} p_{a_{1}}^{\beta}\right. \\
& \left.-i\left(m_{B}+m_{a_{1}}\right) V_{1}^{B \rightarrow a_{1}}\left(m_{\rho}^{2}\right)\left(\epsilon_{a_{1}} \cdot \epsilon_{\rho}\right)+\frac{i V_{2}^{B \rightarrow a_{1}}\left(m_{\rho}^{2}\right)}{\left(m_{B}+m_{a_{1}}\right)}\left(\epsilon_{a_{1}} \cdot p_{B}\right)\left(\epsilon_{\rho} \cdot p_{B}\right)\right) \\
& +m_{a_{1}} f_{a_{1}}\left\{V_{u b} V_{u d}^{*} a_{1}-V_{t b} V_{t d}^{*} \frac{3}{2}\left(-a_{7}+a_{9}+a_{10}\right)\right\}\left(\frac{2 V^{B \rightarrow \rho}\left(m_{a_{1}}^{2}\right)}{\left(m_{B}+m_{\rho}\right)} \epsilon_{\mu \nu \alpha \beta} \epsilon_{a_{1}}^{\mu} \epsilon_{\rho}^{\nu} p_{B}^{\alpha} p_{\rho}^{\beta}\right. \\
& \left.-i\left(m_{B}+m_{\rho}\right) A_{1}^{B \rightarrow \rho}\left(m_{a_{1}}^{2}\right)\left(\epsilon_{\rho} \cdot \epsilon_{a_{1}}\right)+\frac{i A_{2}^{B \rightarrow \rho}\left(m_{a_{1}}^{2}\right)}{\left(m_{B}+m_{\rho}\right)}\left(\epsilon_{\rho} \cdot p_{B}\right)\left(\epsilon_{a_{1}} \cdot p_{B}\right)\right)
\end{aligned}
$$




$$
\begin{aligned}
\mathcal{M}\left(B^{-} \rightarrow a_{1}^{0} \rho^{-}\right)= & -m_{\rho} f_{\rho}\left\{V_{u b} V_{u d}^{*} a_{1}-V_{t b} V_{t d}^{*} \frac{3}{2}\left(a_{7}+a_{9}+a_{10}\right)\right\}\left(\frac{2 A^{B \rightarrow a_{1}}\left(m_{\rho}^{2}\right)}{\left(m_{B}+m_{a_{1}}\right)} \epsilon_{\mu \nu \alpha \beta} \epsilon_{\rho}^{\mu} \epsilon_{a_{1}}^{\nu} p_{B}^{\alpha} p_{a_{1}}^{\beta}\right. \\
& \left.-i\left(m_{B}+m_{a_{1}}\right) V_{1}^{B \rightarrow a_{1}}\left(m_{\rho}^{2}\right)\left(\epsilon_{a_{1}} \cdot \epsilon_{\rho}\right)+\frac{i V_{2}^{B \rightarrow a_{1}}\left(m_{\rho}^{2}\right)}{\left(m_{B}+m_{a_{1}}\right)}\left(\epsilon_{a_{1}} \cdot p_{B}\right)\left(\epsilon_{\rho} \cdot p_{B}\right)\right) \\
& +m_{a_{1}} f_{a_{1}} V_{u b} V_{u d}^{*} a_{2}\left(\frac{2 V^{B \rightarrow \rho}\left(m_{a_{1}}^{2}\right)}{\left(m_{B}+m_{\rho}\right)} \epsilon_{\mu \nu \alpha \beta} \epsilon_{a_{1}}^{\mu} \epsilon_{\rho}^{\nu} p_{B}^{\alpha} p_{\rho}^{\beta}\right. \\
& \left.-i\left(m_{B}+m_{\rho}\right) A_{1}^{B \rightarrow \rho}\left(m_{a_{1}}^{2}\right)\left(\epsilon_{\rho} \cdot \epsilon_{a_{1}}\right)+\frac{i A_{2}^{B \rightarrow \rho}\left(m_{a_{1}}^{2}\right)}{\left(m_{B}+m_{\rho}\right)}\left(\epsilon_{\rho} \cdot p_{B}\right)\left(\epsilon_{a_{1}} \cdot p_{B}\right)\right)
\end{aligned}
$$

$$
\begin{aligned}
\mathcal{M}\left(\bar{B}^{0} \rightarrow a_{1}^{+} K^{*-}\right)= & -m_{K^{*}} f_{K^{*}}\left\{V_{u b} V_{u s}^{*} a_{1}-V_{t b} V_{t s}^{*}\left(a_{4}+a_{10}\right)\right\}\left(\frac{2 A^{B \rightarrow a_{1}}\left(m_{K^{*}}^{2}\right)}{\left(m_{B}+m_{a_{1}}\right)} \epsilon_{\mu \nu \alpha \beta} \epsilon_{K^{*}}^{\mu} \epsilon_{a_{1}}^{\nu} p_{B}^{\alpha} p_{a_{1}}^{\beta}\right. \\
& \left.-i\left(m_{B}+m_{a_{1}}\right) V_{1}^{B \rightarrow a_{1}}\left(m_{K^{*}}^{2}\right)\left(\epsilon_{a_{1}} \cdot \epsilon_{K^{*}}\right)+\frac{i V_{2}^{B \rightarrow a_{1}}\left(m_{K^{*}}^{2}\right)}{\left(m_{B}+m_{a_{1}}\right)}\left(\epsilon_{a_{1}} \cdot p_{B}\right)\left(\epsilon_{K^{*}} \cdot p_{B}\right)\right)
\end{aligned}
$$

$$
\begin{aligned}
\mathcal{M}\left(\bar{B}^{0} \rightarrow a_{1}^{0} \bar{K}^{* 0}\right)= & m_{K^{*}} f_{K^{*}} V_{t b} V_{t s}^{*}\left\{\left(a_{4}-\frac{1}{2} a_{10}\right)\right\}\left(\frac{2 A^{B \rightarrow a_{1}}\left(m_{K^{*}}^{2}\right)}{\left(m_{B}+m_{a_{1}}\right)} \epsilon_{\mu \nu \alpha \beta} \epsilon_{K^{*}}^{\mu} \epsilon_{a_{1}}^{\nu} p_{B}^{\alpha} p_{a_{1}}^{\beta}\right. \\
& \left.-i\left(m_{B}+m_{a_{1}}\right) V_{1}^{B \rightarrow a_{1}}\left(m_{K^{*}}^{2}\right)\left(\epsilon_{a_{1}} \cdot \epsilon_{K^{*}}\right)+\frac{i V_{2}^{B \rightarrow a_{1}}\left(m_{K^{*}}^{2}\right)}{\left(m_{B}+m_{a_{1}}\right)}\left(\epsilon_{a_{1}} \cdot p_{B}\right)\left(\epsilon_{K^{*}} \cdot p_{B}\right)\right) \\
& +m_{a_{1}} f_{a_{1}}\left\{V_{u b} V_{u s}^{*} a_{2}+V_{t b} V_{t s}^{*} \frac{3}{2}\left(a_{7}-a_{9}\right)\right\}\left(\frac{2 V^{B \rightarrow K^{*}}\left(m_{a_{1}}^{2}\right)}{\left(m_{B}+m_{K^{*}}\right)} \epsilon_{\mu \nu \alpha \beta} \epsilon_{a_{1}}^{\mu} \epsilon_{K^{*}}^{\nu} p_{B}^{\alpha} p_{K^{*}}^{\beta}\right. \\
& -i\left(m_{B}+m_{K^{*}} A_{1}^{B \rightarrow K^{*}}\left(m_{a_{1}}^{2}\right)\left(\epsilon_{K^{*}} \cdot \epsilon_{a_{1}}\right)+\frac{i A_{2}^{B \rightarrow K^{*}}\left(m_{a_{1}}^{2}\right)}{\left(m_{B}+m_{K^{*}}\right)}\left(\epsilon_{K^{*}} \cdot p_{B}\right)\left(\epsilon_{a_{1}} \cdot p_{B}\right)\right)
\end{aligned}
$$$$
\mathcal{M}\left(B^{-} \rightarrow a_{1}^{0} K^{*-}\right)=-m_{K^{*}} f_{K^{*}}\left\{V_{u b} V_{u s}^{*} a_{1}-V_{t b} V_{t s}^{*}\left(a_{4}+a_{10}\right)\right\}\left(\frac{2 A^{B \rightarrow a_{1}}\left(m_{K^{*}}^{2}\right)}{\left(m_{B}+m_{a_{1}}\right)} \epsilon_{\mu \nu \alpha \beta} \epsilon_{K^{*}}^{\mu} \epsilon_{a_{1}}^{\nu} p_{B}^{\alpha} p_{a_{1}}^{\beta}\right.
$$$$
\left.-i\left(m_{B}+m_{a_{1}}\right) V_{1}^{B \rightarrow a_{1}}\left(m_{K^{*}}^{2}\right)\left(\epsilon_{a_{1}} \cdot \epsilon_{K^{*}}\right)+\frac{i V_{2}^{B \rightarrow a_{1}}\left(m_{K^{*}}^{2}\right)}{\left(m_{B}+m_{a_{1}}\right)}\left(\epsilon_{a_{1}} \cdot p_{B}\right)\left(\epsilon_{K^{*}} \cdot p_{B}\right)\right)
$$$$
+m_{a_{1}} f_{a_{1}}\left\{V_{u b} V_{u s}^{*} a_{2}+V_{t b} V_{t s}^{*} \frac{3}{2}\left(a_{7}-a_{9}\right)\right\}\left(\frac{2 V^{B \rightarrow K^{*}}\left(m_{a_{1}}^{2}\right)}{\left(m_{B}+m_{K^{*}}\right)} \epsilon_{\mu \nu \alpha \beta} \epsilon_{a_{1}}^{\mu} \epsilon_{K^{*}}^{\nu} p_{B}^{\alpha} p_{K^{*}}^{\beta}\right.
$$$$
\left.-i\left(m_{B}+m_{K^{*}}\right) A_{1}^{B \rightarrow K^{*}}\left(m_{a_{1}}^{2}\right)\left(\epsilon_{K^{*}} \cdot \epsilon_{a_{1}}\right)+\frac{i A_{2}^{B \rightarrow K^{*}}\left(m_{a_{1}}^{2}\right)}{\left(m_{B}+m_{K^{*}}\right)}\left(\epsilon_{K^{*}} \cdot p_{B}\right)\left(\epsilon_{a_{1}} \cdot p_{B}\right)\right)
$$

$$
\begin{aligned}
\mathcal{M}\left(B^{-} \rightarrow a_{1}^{-} \bar{K}^{* 0}\right)= & m_{K^{*}} f_{K^{*}}\left\{V_{t b} V_{t s}^{*}\left(a_{4}-\frac{1}{2} a_{10}\right)\right\}\left(\frac{2 A^{B \rightarrow a_{1}}\left(m_{K^{*}}^{2}\right)}{\left(m_{B}+m_{a_{1}}\right)} \epsilon_{\mu \nu \alpha \beta} \epsilon_{K^{*}}^{\mu} \epsilon_{a_{1}}^{\nu} p_{B}^{\alpha} p_{a_{1}}^{\beta}\right. \\
& \left.-i\left(m_{B}+m_{a_{1}}\right) V_{1}^{B \rightarrow a_{1}}\left(m_{K^{*}}^{2}\right)\left(\epsilon_{a_{1}} \cdot \epsilon_{K^{*}}\right)+\frac{i V_{2}^{B \rightarrow a_{1}}\left(m_{K^{*}}^{2}\right)}{\left(m_{B}+m_{a_{1}}\right)}\left(\epsilon_{a_{1}} \cdot p_{B}\right)\left(\epsilon_{K^{*}} \cdot p_{B}\right)\right)
\end{aligned}
$$




$$
\begin{aligned}
& \mathcal{M}\left(\bar{B}^{0} \rightarrow a_{1}^{0} \omega\right)=-m_{\omega} f_{\omega}\left\{V_{u b} V_{u d}^{*} a_{2}-V_{t b} V_{t d}^{*}\left[2 a_{3}+a_{4}+2 a_{5}+\frac{1}{2}\left(a_{7}+a_{9}-a_{10}\right)\right]\right\}\left(\frac{2 A^{B \rightarrow a_{1}}\left(m_{\omega}^{2}\right)}{\left(m_{B}+m_{a_{1}}\right)} \epsilon_{\mu \nu \alpha \beta} \epsilon_{\omega}^{\mu} \epsilon_{a_{1}}^{\nu} p_{B}^{\alpha} p_{a_{1}}^{\beta}\right. \\
& \left.-i\left(m_{B}+m_{a_{1}}\right) V_{1}^{B \rightarrow a_{1}}\left(m_{\omega}^{2}\right)\left(\epsilon_{a_{1}} \cdot \epsilon_{\omega}\right)+\frac{i V_{2}^{B \rightarrow a_{1}}\left(m_{\omega}^{2}\right)}{\left(m_{B}+m_{a_{1}}\right)}\left(\epsilon_{a_{1}} \cdot p_{B}\right)\left(\epsilon_{\omega} \cdot p_{B}\right)\right) \\
& +m_{a_{1}} f_{a_{1}}\left\{V_{u b} V_{u d}^{*} a_{2}-V_{t b} V_{t d}^{*}\left[-a_{4}-\frac{1}{2}\left(3 a_{7}-3 a_{9}-a_{10}\right)\right]\right\}\left(\frac{2 V^{B \rightarrow \omega}\left(m_{a_{1}}^{2}\right)}{\left(m_{B}+m_{\omega}\right)} \epsilon_{\mu \nu \alpha \beta} \epsilon_{a_{1}}^{\mu} \epsilon_{\omega}^{\nu} p_{B}^{\alpha} p_{\omega}^{\beta}\right. \\
& \left.-i\left(m_{B}+m_{\omega}\right) A_{1}^{B \rightarrow \omega}\left(m_{a_{1}}^{2}\right)\left(\epsilon_{\omega} \cdot \epsilon_{a_{1}}\right)+\frac{i A_{2}^{B \rightarrow \omega}\left(m_{a_{1}}^{2}\right)}{\left(m_{B}+m_{\omega}\right)}\left(\epsilon_{\omega} \cdot p_{B}\right)\left(\epsilon_{a_{1}} \cdot p_{B}\right)\right) \\
& \mathcal{M}\left(B^{-} \rightarrow a_{1}^{-} \omega\right)=-m_{\omega} f_{\omega}\left\{V_{u b} V_{u d}^{*} a_{2}-V_{t b} V_{t d}^{*}\left[2 a_{3}+a_{4}+2 a_{5}+\frac{1}{2}\left(a_{7}+a_{9}-a_{10}\right)\right]\right\}\left(\frac{2 A^{B \rightarrow a_{1}}\left(m_{\omega}^{2}\right)}{\left(m_{B}+m_{a_{1}}\right)} \epsilon_{\mu \nu \alpha \beta} \epsilon_{\omega}^{\mu} \epsilon_{a_{1}}^{\nu} p_{B}^{\alpha} p_{a_{1}}^{\beta}\right. \\
& \left.-i\left(m_{B}+m_{a_{1}}\right) V_{1}^{B \rightarrow a_{1}}\left(m_{\omega}^{2}\right)\left(\epsilon_{a_{1}} \cdot \epsilon_{\omega}\right)+\frac{i V_{2}^{B \rightarrow a_{1}}\left(m_{\omega}^{2}\right)}{\left(m_{B}+m_{a_{1}}\right)}\left(\epsilon_{a_{1}} \cdot p_{B}\right)\left(\epsilon_{\omega} \cdot p_{B}\right)\right) \\
& +m_{a_{1}} f_{a_{1}}\left\{V_{u b} V_{u d}^{*} a_{1}-V_{t b} V_{t d}^{*}\left(a_{4}+a_{10}\right)\right\}\left(\frac{2 V^{B \rightarrow \omega}\left(m_{a_{1}}^{2}\right)}{\left(m_{B}+m_{\omega}\right)} \epsilon_{\mu \nu \alpha \beta} \epsilon_{a_{1}}^{\mu} \epsilon_{\omega}^{\nu} p_{B}^{\alpha} p_{\omega}^{\beta}\right. \\
& \left.-i\left(m_{B}+m_{\omega}\right) A_{1}^{B \rightarrow \omega}\left(m_{a_{1}}^{2}\right)\left(\epsilon_{\omega} \cdot \epsilon_{a_{1}}\right)+\frac{i A_{2}^{B \rightarrow \omega}\left(m_{a_{1}}^{2}\right)}{\left(m_{B}+m_{\omega}\right)}\left(\epsilon_{\omega} \cdot p_{B}\right)\left(\epsilon_{a_{1}} \cdot p_{B}\right)\right) \\
& \mathcal{M}\left(\bar{B}^{0} \rightarrow a_{1}^{0} \phi\right)=m_{\phi} f_{\phi} V_{t b} V_{t d}^{*}\left\{\left[a_{3}+a_{5}-\frac{1}{2}\left(a_{7}+a_{9}\right)\right]\right\}\left(\frac{2 A^{B \rightarrow a_{1}}\left(m_{\phi}^{2}\right)}{\left(m_{B}+m_{a_{1}}\right)} \epsilon_{\mu \nu \alpha \beta} \epsilon_{\phi}^{\mu} \epsilon_{a_{1}}^{\nu} p_{B}^{\alpha} p_{a_{1}}^{\beta}\right. \\
& \left.-i\left(m_{B}+m_{a_{1}}\right) V_{1}^{B \rightarrow a_{1}}\left(m_{\phi}^{2}\right)\left(\epsilon_{a_{1}} \cdot \epsilon_{\phi}\right)+\frac{i V_{2}^{B \rightarrow a_{1}}\left(m_{\phi}^{2}\right)}{\left(m_{B}+m_{a_{1}}\right)}\left(\epsilon_{a_{1}} \cdot p_{B}\right)\left(\epsilon_{\phi} \cdot p_{B}\right)\right) \\
& \mathcal{M}\left(B^{-} \rightarrow a_{1}^{-} \phi\right)=-\sqrt{2} \mathcal{M}\left(\bar{B}^{0} \rightarrow a_{1}^{0} \phi\right) \\
& \mathcal{M}\left(\bar{B}^{0} \rightarrow f_{1} \rho^{0}\right)=-m_{\rho} f_{\rho}\left\{V_{u b} V_{u d}^{*} a_{2}-V_{t b} V_{t d}^{*}\left[-a_{4}+\frac{1}{2}\left(3 a_{7}+3 a_{9}+a_{10}\right)\right]\right\}\left(\frac{2 A^{B \rightarrow f_{1}}\left(m_{\rho}^{2}\right)}{\left(m_{B}+m_{f_{1}}\right)} \epsilon_{\mu \nu \alpha \beta} \epsilon_{\rho}^{\mu} \epsilon_{f_{1}}^{\nu} p_{B}^{\alpha} p_{f_{1}}^{\beta}\right. \\
& \left.-i\left(m_{B}+m_{f_{1}}\right) V_{1}^{B \rightarrow f_{1}}\left(m_{\rho}^{2}\right)\left(\epsilon_{f_{1}} \cdot \epsilon_{\rho}\right)+\frac{i V_{2}^{B \rightarrow f_{1}}\left(m_{\rho}^{2}\right)}{\left(m_{B}+m_{f_{1}}\right)}\left(\epsilon_{f_{1}} \cdot p_{B}\right)\left(\epsilon_{\rho} \cdot p_{B}\right)\right) \\
& +m_{f_{1}} f_{f_{1}}\left\{V_{u b} V_{u d}^{*} a_{2}-V_{t b} V_{t d}^{*}\left[2 a_{3}+a_{4}-2 a_{5}-\frac{1}{2}\left(a_{7}-a_{9}+a_{10}\right)\right]\right\}\left(\frac{2 V^{B \rightarrow \rho}\left(m_{f_{1}}^{2}\right)}{\left(m_{B}+m_{\rho}\right)} \epsilon_{\mu \nu \alpha \beta} \epsilon_{f_{1}}^{\mu} \epsilon_{\rho}^{\nu} p_{B}^{\alpha} p_{\rho}^{\beta}\right. \\
& \left.-i\left(m_{B}+m_{\rho}\right) A_{1}^{B \rightarrow \rho}\left(m_{f_{1}}^{2}\right)\left(\epsilon_{\rho} \cdot \epsilon_{f_{1}}\right)+\frac{i A_{2}^{B \rightarrow \rho}\left(m_{f_{1}}^{2}\right)}{\left(m_{B}+m_{\rho}\right)}\left(\epsilon_{\rho} \cdot p_{B}\right)\left(\epsilon_{f_{1}} \cdot p_{B}\right)\right)
\end{aligned}
$$




$$
\begin{aligned}
& \mathcal{M}\left(B^{-} \rightarrow f_{1} \rho^{-}\right)=-m_{\rho}^{-} f_{\rho}\left\{V_{u b} V_{u d}^{*} a_{1}-V_{t b} V_{t d}^{*}\left(a_{4}+a_{10}\right)\right\}\left(\frac{2 A^{B \rightarrow f_{1}}\left(m_{\rho}^{2}\right)}{\left(m_{B}+m_{f_{1}}\right)} \epsilon_{\mu \nu \alpha \beta} \epsilon_{\rho}^{\mu} \epsilon_{f_{1}}^{\nu} p_{B}^{\alpha} p_{f_{1}}^{\beta}\right. \\
& \left.-i\left(m_{B}+m_{f_{1}}\right) V_{1}^{B \rightarrow f_{1}}\left(m_{\rho^{-}}^{2}\right)\left(\epsilon_{f_{1}} \cdot \epsilon_{\rho}\right)+\frac{i V_{2}^{B \rightarrow f_{1}}\left(m_{\rho}^{2}\right)}{\left(m_{B}+m_{f_{1}}\right)}\left(\epsilon_{f_{1}} \cdot p_{B}\right)\left(\epsilon_{\rho} \cdot p_{B}\right)\right) \\
& +m_{f_{1}} f_{f_{1}}\left\{V_{u b} V_{u d}^{*} a_{2}-V_{t b} V_{t d}^{*}\left[2 a_{3}+a_{4}-2 a_{5}-\frac{1}{2}\left(a_{7}-a_{9}+a_{10}\right)\right]\right\}\left(\frac{2 V^{B \rightarrow \rho}\left(m_{f_{1}}^{2}\right)}{\left(m_{B}+m_{\rho}\right)} \epsilon_{\mu \nu \alpha \beta} \epsilon_{f_{1}}^{\mu} \epsilon_{\rho}^{\nu} p_{B}^{\alpha} p_{\rho}^{\beta}\right. \\
& \left.-i\left(m_{B}+m_{\rho}\right) A_{1}^{B \rightarrow \rho}\left(m_{f_{1}}^{2}\right)\left(\epsilon_{\rho} \cdot \epsilon_{f_{1}}\right)+\frac{i A_{2}^{B \rightarrow \rho}\left(m_{f_{1}}^{2}\right)}{\left(m_{B}+m_{\rho}\right)}\left(\epsilon_{\rho} \cdot p_{B}\right)\left(\epsilon_{f_{1}} \cdot p_{B}\right)\right) \\
& \mathcal{M}\left(\bar{B}^{0} \rightarrow f_{1} \bar{K}^{* 0}\right)=m_{K^{*}} f_{K^{*}} V_{t b} V_{t s}^{*}\left\{\left(a_{4}-\frac{1}{2} a_{10}\right)\right\}\left(\frac{2 A^{B \rightarrow f_{1}}\left(m_{K^{*}}^{2}\right)}{\left(m_{B}+m_{f_{1}}\right)} \epsilon_{\mu \nu \alpha \beta} \epsilon_{K^{*}}^{\mu} \epsilon_{f_{1}}^{\nu} p_{B}^{\alpha} p_{f_{1}}^{\beta}\right. \\
& \left.-i\left(m_{B}+m_{f_{1}}\right) V_{1}^{B \rightarrow f_{1}}\left(m_{K^{*}}^{2}\right)\left(\epsilon_{f_{1}} \cdot \epsilon_{K^{*}}\right)+\frac{i V_{2}^{B \rightarrow f_{1}}\left(m_{K^{*}}^{2}\right)}{\left(m_{B}+m_{f_{1}}\right)}\left(\epsilon_{f_{1}} \cdot p_{B}\right)\left(\epsilon_{K^{*}} \cdot p_{B}\right)\right) \\
& +m_{f_{1}} f_{f_{1}}\left\{V_{u b} V_{u s}^{*} a_{2}-V_{t b} V_{t s}^{*}\left[2 a_{3}-2 a_{5}-\frac{1}{2}\left(a_{7}-a_{9}\right)\right]\right\}\left(\frac{2 V^{B \rightarrow K^{*}\left(m_{f_{1}}^{2}\right)}}{\left(m_{B}+m_{K^{*}}\right)} \epsilon_{\mu \nu \alpha \beta} \epsilon_{f_{1}}^{\mu} \epsilon_{K^{*}}^{\nu} p_{B}^{\alpha} p_{K^{*}}^{\beta}\right. \\
& \left.-i\left(m_{B}+m_{K^{*}}\right) A_{1}^{B \rightarrow K^{*}}\left(m_{f_{1}}^{2}\right)\left(\epsilon_{K^{*}} \cdot \epsilon_{f_{1}}\right)+\frac{i A_{2}^{B \rightarrow K^{*}}\left(m_{f_{1}}^{2}\right)}{\left(m_{B}+m_{K^{*}}\right)}\left(\epsilon_{K^{*}} \cdot p_{B}\right)\left(\epsilon_{f_{1}} \cdot p_{B}\right)\right) \\
& \mathcal{M}\left(B^{-} \rightarrow f_{1} K^{*-}\right)=-m_{K^{*}} f_{K^{*}}\left\{V_{u b} V_{u s}^{*} a_{1}-V_{t b} V_{t s}^{*}\left(a_{4}+a_{10}\right)\right\}\left(\frac{2 A^{B \rightarrow f_{1}}\left(m_{K^{*}}^{2}\right)}{\left(m_{B}+m_{f_{1}}\right)} \epsilon_{\mu \nu \alpha \beta} \epsilon_{K^{*}}^{\mu} \epsilon_{f_{1}}^{\nu} p_{B}^{\alpha} p_{f_{1}}^{\beta}\right. \\
& \left.-i\left(m_{B}+m_{f_{1}}\right) V_{1}^{B \rightarrow f_{1}}\left(m_{K^{*}}^{2}\right)\left(\epsilon_{f_{1}} \cdot \epsilon_{K^{*}}\right)+\frac{i V_{2}^{B \rightarrow f_{1}}\left(m_{K^{*}}^{2}\right)}{\left(m_{B}+m_{f_{1}}\right)}\left(\epsilon_{f_{1}} \cdot p_{B}\right)\left(\epsilon_{K^{*}} \cdot p_{B}\right)\right) \\
& +m_{f_{1}} f_{f_{1}}\left\{V_{u b} V_{u s}^{*} a_{2}-V_{t b} V_{t s}^{*}\left[2 a_{3}-2 a_{5}-\frac{1}{2}\left(a_{7}-a_{9}\right)\right]\right\}\left(\frac{2 V^{B \rightarrow K^{*}}\left(m_{f_{1}}^{2}\right)}{\left(m_{B}+m_{K^{*}}\right)} \epsilon_{\mu \nu \alpha \beta} \epsilon_{f_{1}}^{\mu} \epsilon_{K^{*}}^{\nu} p_{B}^{\alpha} p_{K^{*}}^{\beta}\right. \\
& \left.-i\left(m_{B}+m_{K^{*}}\right) A_{1}^{B \rightarrow K^{*}}\left(m_{f_{1}}^{2}\right)\left(\epsilon_{K^{*}} \cdot \epsilon_{f_{1}}\right)+\frac{i A_{2}^{B \rightarrow K^{*}}\left(m_{f_{1}}^{2}\right)}{\left(m_{B}+m_{K^{*}}\right)}\left(\epsilon_{K^{*}} \cdot p_{B}\right)\left(\epsilon_{f_{1}} \cdot p_{B}\right)\right) \\
& \mathcal{M}\left(\bar{B}^{0} \rightarrow f_{1} \omega\right)=-m_{\omega} f_{\omega}\left\{V_{u b} V_{u d}^{*} a_{2}-V_{t b} V_{t d}^{*}\left[2 a_{3}+a_{4}+2 a_{5}+\frac{1}{2}\left(a_{7}+a_{9}-a_{10}\right)\right]\right\}\left(\frac{2 A^{B \rightarrow f_{1}}\left(m_{\omega}^{2}\right)}{\left(m_{B}+m_{f_{1}}\right)} \epsilon_{\mu \nu \alpha \beta} \epsilon_{\omega}^{\mu} \epsilon_{f_{1}}^{\nu} p_{B}^{\alpha} p_{f_{1}}^{\beta}\right. \\
& \left.-i\left(m_{B}+m_{f_{1}}\right) V_{1}^{B \rightarrow f_{1}}\left(m_{\omega}^{2}\right)\left(\epsilon_{f_{1}} \cdot \epsilon_{\omega}\right)+\frac{i V_{2}^{B \rightarrow f_{1}}\left(m_{\omega}^{2}\right)}{\left(m_{B}+m_{f_{1}}\right)}\left(\epsilon_{f_{1}} \cdot p_{B}\right)\left(\epsilon_{\omega} \cdot p_{B}\right)\right) \\
& +m_{f_{1}} f_{f_{1}}\left\{V_{u b} V_{u d}^{*} a_{2}-V_{t b} V_{t d}^{*}\left[2 a_{3}+a_{4}-2 a_{5}-\frac{1}{2}\left(a_{7}-a_{9}+a_{10}\right)\right]\right\}\left(\frac{2 V^{B \rightarrow \omega}\left(m_{f_{1}}^{2}\right)}{\left(m_{B}+m_{\omega}\right)} \epsilon_{\mu \nu \alpha \beta} \epsilon_{f_{1}}^{\mu} \epsilon_{\omega}^{\nu} p_{B}^{\alpha} p_{\omega}^{\beta}\right. \\
& \left.-i\left(m_{B}+m_{\omega}\right) A_{1}^{B \rightarrow \omega}\left(m_{f_{1}}^{2}\right)\left(\epsilon_{\omega} \cdot \epsilon_{f_{1}}\right)+\frac{i A_{2}^{B \rightarrow \omega}\left(m_{f_{1}}^{2}\right)}{\left(m_{B}+m_{\omega}\right)}\left(\epsilon_{\omega} \cdot p_{B}\right)\left(\epsilon_{f_{1}} \cdot p_{B}\right)\right) \\
& \mathcal{M}\left(\bar{B}^{0} \rightarrow f_{1} \phi\right)=m_{\phi} f_{\phi}\left\{V_{t b} V_{t d}^{*}\left[a_{3}+a_{5}-\frac{1}{2}\left(a_{7}+a_{9}\right)\right]\right\}\left(\frac{2 A^{B \rightarrow f_{1}}\left(m_{\phi}^{2}\right)}{\left(m_{B}+m_{f_{1}}\right)} \epsilon_{\mu \nu \alpha \beta} \epsilon_{\phi}^{\mu} \epsilon_{f_{1}}^{\nu} p_{B}^{\alpha} p_{f_{1}}^{\beta}\right. \\
& \left.-i\left(m_{B}+m_{f_{1}}\right) V_{1}^{B \rightarrow f_{1}}\left(m_{\phi}^{2}\right)\left(\epsilon_{f_{1}} \cdot \epsilon_{\phi}\right)+\frac{i V_{2}^{B \rightarrow f_{1}}\left(m_{\phi}^{2}\right)}{\left(m_{B}+m_{f_{1}}\right)}\left(\epsilon_{f_{1}} \cdot p_{B}\right)\left(\epsilon_{\phi} \cdot p_{B}\right)\right)
\end{aligned}
$$




$$
\begin{aligned}
\mathcal{M}\left(\bar{B}^{0} \rightarrow K_{1} \rho^{+}\right)= & m_{K_{1}} f_{K_{1}}\left\{V_{u b} V_{u s}^{*} a_{1}-V_{t b} V_{t s}^{*}\left(a_{4}+a_{10}\right)\right\}\left(\frac{2 V^{B \rightarrow \rho}\left(m_{K_{1}}^{2}\right)}{\left(m_{B}+m_{\rho}\right)} \epsilon_{\mu \nu \alpha \beta} \epsilon_{K_{1}}^{\mu} \epsilon_{\rho}^{\nu} p_{B}^{\alpha} p_{\rho}^{\beta}\right. \\
& \left.-i\left(m_{B}+m_{\rho}\right) A_{1}^{B \rightarrow \rho}\left(m_{K_{1}}^{2}\right)\left(\epsilon_{\rho} \cdot \epsilon_{K_{1}}\right)+\frac{i A_{2}^{B \rightarrow \rho}\left(m_{K_{1}}^{2}\right)}{\left(m_{B}+m_{\rho}\right)}\left(\epsilon_{\rho} \cdot p_{B}\right)\left(\epsilon_{K_{1}} \cdot p_{B}\right)\right) \\
\mathcal{M}\left(\bar{B}^{0} \rightarrow \bar{K}_{1}^{0} \rho^{0}\right)= & -m_{\rho} f_{\rho}\left\{V_{u b} V_{u s}^{*} a_{2}-V_{t b} V_{t s}^{*} \frac{3}{2}\left(a_{7}+a_{9}\right)\right\}\left(\frac{2 A^{B \rightarrow K_{1}}\left(m_{\rho}^{2}\right)}{\left(m_{B}+m_{K_{1}}\right)} \epsilon_{\mu \nu \alpha \beta} \epsilon_{\rho}^{\mu} \epsilon_{K_{1}}^{\nu} p_{B}^{\alpha} p_{K_{1}}^{\beta}\right. \\
& \left.-i\left(m_{B}+m_{K_{1}}\right) V_{1}^{B \rightarrow K_{1}}\left(m_{\rho}^{2}\right)\left(\epsilon_{K_{1}} \cdot \epsilon_{\rho}\right)+\frac{i V_{2}^{B \rightarrow K_{1}}\left(m_{\rho}^{2}\right)}{\left(m_{B}+m_{K_{1}}\right)}\left(\epsilon_{K_{1}} \cdot p_{B}\right)\left(\epsilon_{\rho} \cdot p_{B}\right)\right) \\
& -m_{K_{1}} f_{K_{1}}\left\{V_{t b} V_{t s}^{*}\left(a_{4}-\frac{1}{2} a_{10}\right)\right\}\left(\frac{2 V^{B \rightarrow \rho}\left(m_{K_{1}}^{2}\right)}{\left(m_{B}+m_{\rho}\right)} \epsilon_{\mu \nu \alpha \beta} \epsilon_{K_{1}}^{\mu} \epsilon_{\rho}^{\nu} p_{B}^{\alpha} p_{\rho}^{\beta}\right. \\
& \left.-i\left(m_{B}+m_{\rho}\right) A_{1}^{B \rightarrow \rho}\left(m_{K_{1}}^{2}\right)\left(\epsilon_{\rho}^{0} \cdot \epsilon_{K_{1}}\right)+\frac{i A_{2}^{B \rightarrow \rho}\left(m_{K_{1}}^{2}\right)}{\left(m_{B}+m_{\rho}\right)}\left(\epsilon_{\rho} \cdot p_{B}\right)\left(\epsilon_{K_{1}} \cdot p_{B}\right)\right)
\end{aligned}
$$

$$
\begin{aligned}
\mathcal{M}\left(B^{-} \rightarrow K_{1}^{-} \rho^{0}\right)= & -m_{\rho} f_{\rho}\left\{V_{u b} V_{u s}^{*} a_{2}-V_{t b} V_{t s}^{*} \frac{3}{2}\left(a_{7}+a_{9}\right)\right\}\left(\frac{2 A^{B \rightarrow K_{1}}\left(m_{\rho}^{2}\right)}{\left(m_{B}+m_{K_{1}}\right)} \epsilon_{\mu \nu \alpha \beta} \epsilon_{\rho}^{\mu} \epsilon_{K_{1}}^{\nu} p_{B}^{\alpha} p_{K_{1}}^{\beta}\right. \\
& \left.-i\left(m_{B}+m_{K_{1}}\right) V_{1}^{B \rightarrow K_{1}}\left(m_{\rho}^{2}\right)\left(\epsilon_{K_{1}} \cdot \epsilon_{\rho}\right)+\frac{i V_{2}^{B \rightarrow K_{1}}\left(m_{\rho}^{2}\right)}{\left(m_{B}+m_{K_{1}}\right)}\left(\epsilon_{K_{1}} \cdot p_{B}\right)\left(\epsilon_{\rho} \cdot p_{B}\right)\right) \\
& +m_{K_{1}} f_{K_{1}}\left\{V_{u b} V_{u s}^{*} a_{1}-V_{t b} V_{t s}^{*}\left(a_{4}+a_{10}\right)\right\}\left(\frac{2 V^{B \rightarrow \rho}\left(m_{K_{1}}^{2}\right)}{\left(m_{B}+m_{\rho}\right)} \epsilon_{\mu \nu \alpha \beta} \epsilon_{K_{1}}^{\mu} \epsilon_{\rho}^{\nu} p_{B}^{\alpha} p_{\rho}^{\beta}\right. \\
& \left.-i\left(m_{B}+m_{\rho}\right) A_{1}^{B \rightarrow \rho}\left(m_{K_{1}}\right)\left(\epsilon_{\rho} \cdot \epsilon_{K_{1}}\right)+\frac{i A_{2}^{B \rightarrow \rho}\left(m_{K_{1}}^{2}\right)}{\left(m_{B}+m_{\rho}\right)}\left(\epsilon_{\rho} \cdot p_{B}\right)\left(\epsilon_{K_{1}} \cdot p_{B}\right)\right)
\end{aligned}
$$

$$
\begin{aligned}
\mathcal{M}\left(B^{-} \rightarrow \bar{K}_{1}^{0} \rho^{-}\right)= & -m_{K_{1}} f_{K_{1}}\left\{V_{t b} V_{t s}^{*}\left(a_{4}-\frac{1}{2} a_{10}\right)\right\}\left(\frac{2 V^{B \rightarrow \rho}\left(m_{K_{1}^{0}}^{2}\right)}{\left(m_{B}+m_{\rho}\right)} \epsilon_{\mu \nu \alpha \beta} \epsilon_{K_{1}}^{\mu} \epsilon_{\rho}^{\nu} p_{B}^{\alpha} p_{\rho}^{\beta}\right. \\
& \left.-i\left(m_{B}+m_{\rho}\right) A_{1}^{B \rightarrow \rho}\left(m_{K_{1}}^{2}\right)\left(\epsilon_{\rho} \cdot \epsilon_{K_{1}}\right)+\frac{i A_{2}^{B \rightarrow \rho}\left(m_{K_{1}}^{2}\right)}{\left(m_{B}+m_{\rho}\right)}\left(\epsilon_{\rho} \cdot p_{B}\right)\left(\epsilon_{K_{1}} \cdot p_{B}\right)\right)
\end{aligned}
$$

$$
\begin{aligned}
\mathcal{M}\left(\bar{B}^{0} \rightarrow \bar{K}_{1}^{0} \omega\right)= & -m_{\omega} f_{\omega}\left\{V_{u b} V_{u s}^{*} a_{2}-V_{t b} V_{t s}^{*}\left[2\left(a_{3}+a_{5}\right)+\frac{1}{2}\left(a_{7}+a_{9}\right)\right]\right\}\left(\frac{2 A^{B \rightarrow K_{1}}\left(m_{\omega}^{2}\right)}{\left(m_{B}+m_{K_{1}}\right)} \epsilon_{\mu \nu \alpha \beta} \epsilon_{\omega}^{\mu} \epsilon_{K_{1}}^{\nu} p_{B}^{\alpha} p_{K_{1}}^{\beta}\right. \\
& \left.-i\left(m_{B}+m_{K_{1}}\right) V_{1}^{B \rightarrow K_{1}}\left(m_{\omega}^{2}\right)\left(\epsilon_{K_{1}} \cdot \epsilon_{\omega}\right)+\frac{i V_{2}^{B \rightarrow K_{1}}\left(m_{\omega}^{2}\right)}{\left(m_{B}+m_{K_{1}}\right)}\left(\epsilon_{K_{1}} \cdot p_{B}\right)\left(\epsilon_{\omega} \cdot p_{B}\right)\right) \\
& -m_{K_{1}} f_{K_{1}} V_{t b} V_{t s}^{*}\left\{\left(a_{4}-\frac{1}{2} a_{10}\right)\right\}\left(\frac{2 V^{B \rightarrow \omega}\left(m_{K_{1}}^{2}\right)}{\left(m_{B}+m_{\omega}\right)} \epsilon_{\mu \nu \alpha \beta} \epsilon_{K_{1}}^{\mu} \epsilon_{\omega}^{\nu} p_{B}^{\alpha} p_{\omega}^{\beta}\right. \\
& \left.-i\left(m_{B}+m_{\omega}\right) A_{1}^{B \rightarrow \omega}\left(m_{K_{1}}^{2}\right)\left(\epsilon_{\omega} \cdot \epsilon_{K_{1}}\right)+\frac{i A_{2}^{B \rightarrow \omega}\left(m_{K_{1}}^{2}\right)}{\left(m_{B}+m_{\omega}\right)}\left(\epsilon_{\omega} \cdot p_{B}\right)\left(\epsilon_{K_{1}} \cdot p_{B}\right)\right)
\end{aligned}
$$




$$
\begin{aligned}
\mathcal{M}\left(B^{-} \rightarrow K_{1}^{-} \omega\right)= & -m_{\omega} f_{\omega}\left\{V_{u b} V_{u s}^{*} a_{2}-V_{t b} V_{t s}^{*}\left[2\left(a_{3}+a_{5}\right)+\frac{1}{2}\left(a_{7}+a_{9}\right)\right]\right\}\left(\frac{2 A^{B \rightarrow K_{1}}\left(m_{\omega}^{2}\right)}{\left(m_{B}+m_{K_{1}}\right)} \epsilon_{\mu \nu \alpha \beta} \epsilon_{\omega}^{\mu} \epsilon_{K_{1}}^{\nu} p_{B}^{\alpha} p_{K_{1}}^{\beta}\right. \\
& \left.-i\left(m_{B}+m_{K_{1}}\right) V_{1}^{B \rightarrow K_{1}}\left(m_{\omega}^{2}\right)\left(\epsilon_{K_{1}} \cdot \epsilon_{\omega}\right)+\frac{i V_{2}^{B \rightarrow K_{1}}\left(m_{\omega}^{2}\right)}{\left(m_{B}+m_{K_{1}}\right)}\left(\epsilon_{K_{1}} \cdot p_{B}\right)\left(\epsilon_{\omega} \cdot p_{B}\right)\right) \\
& +m_{K_{1}} f_{K_{1}}\left\{V_{u b} V_{u s}^{*} a_{1}-V_{t b} V_{t s}^{*}\left(a_{4}+a_{10}\right)\right\}\left(\frac{2 V^{B \rightarrow \omega}\left(m_{K_{1}}^{2}\right)}{\left(m_{B}+m_{\omega}\right)} \epsilon_{\mu \nu \alpha \beta} \epsilon_{K_{1}}^{\mu} \epsilon_{\omega}^{\nu} p_{B}^{\alpha} p_{\omega}^{\beta}\right. \\
- & \left.i\left(m_{B}+m_{\omega}\right) A_{1}^{B \rightarrow \omega}\left(m_{K_{1}}^{2}\right)\left(\epsilon_{\omega} \cdot \epsilon_{K_{1}}\right)+\frac{i A_{2}^{B \rightarrow \omega}\left(m_{K_{1}}^{2}\right)}{\left(m_{B}+m_{\omega}\right)}\left(\epsilon_{\omega} \cdot p_{B}\right)\left(\epsilon_{K_{1}} \cdot p_{B}\right)\right) \\
\mathcal{M}\left(\bar{B}^{0} \rightarrow \bar{K}_{1}^{0} \phi\right)= & m_{\phi} f_{\phi}\left\{V_{t b} V_{t s}^{*}\left[a_{3}+a_{4}+a_{5}-\frac{1}{2}\left(a_{7}+a_{9}+a_{10}\right)\right]\right\}\left(\frac{2 A^{B \rightarrow K_{1}}\left(m_{\phi}^{2}\right)}{\left(m_{B}+m_{K_{1}}\right)} \epsilon_{\mu \nu \alpha \beta} \epsilon_{\phi}^{\mu} \epsilon_{K_{1}}^{\nu} p_{B}^{\alpha} p_{K_{1}}^{\beta}\right. \\
& \left.-i\left(m_{B}+m_{K_{1}}\right) V_{1}^{B \rightarrow K_{1}}\left(m_{\phi}^{2}\right)\left(\epsilon_{K_{1}} \cdot \epsilon_{\phi}\right)+\frac{i V_{2}^{B \rightarrow K_{1}}\left(m_{\phi}^{2}\right)}{\left(m_{B}+m_{K_{1}}\right)}\left(\epsilon_{K_{1}} \cdot p_{B}\right)\left(\epsilon_{\phi} \cdot p_{B}\right)\right) \\
\mathcal{M}\left(B^{0} \rightarrow K_{1}^{0} \bar{K}^{* 0}\right)= & m_{K^{*}} f_{K^{*}}\left\{V_{t b} V_{t d}^{*}\left(a_{4}-\frac{1}{2} a_{10}\right)\right\}\left(\frac{2 A^{B \rightarrow K_{1}}\left(m_{K^{*}}^{2}\right)}{\left(m_{B}+m_{K_{1}}\right)} \epsilon_{\mu \nu \alpha \beta} \epsilon_{K^{*}}^{\mu} \epsilon_{K_{1}}^{\nu} p_{B}^{\alpha} p_{K_{1}}^{\beta}\right. \\
& \left.-i\left(m_{B}+m_{K_{1}}\right) V_{1}^{B \rightarrow K_{1}}\left(m_{K^{*}}^{2}\right)\left(\epsilon_{K_{1}} \cdot \epsilon_{K *}\right)+\frac{i V_{2}^{B \rightarrow K_{1}}\left(m_{K^{*}}^{2}\right)}{\left(m_{B}+m_{K_{1}}\right)}\left(\epsilon_{K_{1}} \cdot p_{B}\right)\left(\epsilon_{K^{*}} \cdot p_{B}\right)\right)(\mathrm{B} 27)
\end{aligned}
$$

\section{APPENDIX C: MATRIX ELEMENTS FOR $B$ DECAYS TO TWO AXIAL MESONS}

$$
\begin{gathered}
\mathcal{M}\left(\bar{B}^{0} \rightarrow a_{1}^{-} a_{1}^{+}\right)=m_{a_{1}} f_{a_{1}}\left\{V_{u b} V_{u d}^{*} a_{1}-V_{t b} V_{t d}^{*}\left(a_{4}+a_{10}\right)\right\}\left(\frac{2 A^{B \rightarrow a_{1}}\left(m_{a_{1}}^{2}\right)}{\left(m_{B}+m_{a_{1}}\right)} \epsilon_{\mu \nu \alpha \beta} \epsilon_{a_{1}^{+}}^{\mu} \epsilon_{a_{1}^{-}}^{\nu} p_{B}^{\alpha} p_{a_{1}}^{\beta}\right. \\
\left.-i\left(m_{B}+m_{a_{1}}\right) V_{1}^{B \rightarrow a_{1}}\left(m_{a_{1}}^{2}\right)\left(\epsilon_{a_{1}} \cdot \epsilon_{a_{1}}\right)+\frac{i V_{2}^{B \rightarrow a_{1}}\left(m_{a_{1}}^{2}\right)}{\left(m_{B}+m_{a_{1}}\right)}\left(\epsilon_{a_{1}} \cdot p_{B}\right)\left(\epsilon_{a_{1}} \cdot p_{B}\right)\right) \\
\mathcal{M}\left(\bar{B}^{0} \rightarrow a_{1}^{0} a_{1}^{0}\right)=m_{a_{1}} f_{a_{1}}\left\{V_{u b} V_{u d}^{*} 2 a_{2}-2 V_{t b} V_{t d}^{*}\left[-a_{4}-\frac{1}{2}\left(3 a_{7}-3 a_{9}-a_{10}\right)\right]\right\}\left(\frac{2 A^{B \rightarrow a_{1}}\left(m_{a_{1}}^{2}\right)}{\left(m_{B}+m_{a_{1}}\right)} \epsilon_{\mu \nu \alpha \beta} \epsilon_{a_{1}}^{\mu} \epsilon_{a_{1}^{0}}^{\nu} p_{B}^{\alpha} p_{a_{1}}^{\beta}\right. \\
\left.-i\left(m_{B}+m_{a_{1}}\right) V_{1}^{B \rightarrow a_{1}}\left(m_{a_{1}}^{2}\right)\left(\epsilon_{a_{1}} \cdot \epsilon_{a_{1}}\right)+\frac{i V_{2}^{B \rightarrow a_{1}}\left(m_{a_{1}}^{2}\right)}{\left(m_{B}+m_{a_{1}}\right)}\left(\epsilon_{a_{1}} \cdot p_{B}\right)\left(\epsilon_{a_{1}} \cdot p_{B}\right)\right) \\
\mathcal{M}\left(B^{-} \rightarrow a_{1}^{-} a_{1}^{0}\right)=m_{a_{1}} f_{a_{1}}\left\{V_{u b} V_{u d}^{*} a_{1}-V_{t b} V_{t d}^{*} \frac{3}{2}\left(-a_{7}+a_{9}+a_{10}\right)\right\}\left(\frac{2 A^{B \rightarrow a_{1}}\left(m_{a_{1}}^{2}\right)}{\left(m_{B}+m_{a_{1}}\right)} \epsilon_{\mu \nu \alpha \beta} \epsilon_{a_{1}^{\mu}}^{\mu} \epsilon_{a_{1}^{+}}^{\nu} p_{B}^{\alpha} p_{a_{1}}^{\beta}\right. \\
\left.-i\left(m_{B}+m_{a_{1}}\right) V_{1}^{B \rightarrow a_{1}}\left(m_{a_{1}}^{2}\right)\left(\epsilon_{a_{1}} \cdot \epsilon_{a_{1}}\right)+\frac{i V_{2}^{B \rightarrow a_{1}}\left(m_{a_{1}}^{2}\right)}{\left(m_{B}+m_{a_{1}}\right)}\left(\epsilon_{a_{1}} \cdot p_{B}\right)\left(\epsilon_{a_{1}} \cdot p_{B}\right)\right) \\
+m_{a_{1}} f_{a_{1}}\left\{V_{u b} V_{u d}^{*} a_{2}\right\}\left(\frac{2 A^{B \rightarrow a_{1}}\left(m_{a_{1}}^{2}\right)}{\left(m_{B}+m_{a_{1}}\right)} \epsilon_{\mu \nu \alpha \beta} \epsilon_{a_{1}^{+}}^{\mu} \epsilon_{a_{1}^{-}}^{\nu} p_{B}^{\alpha} p_{a_{1}}^{\beta}\right. \\
\left.-i\left(m_{B}+m_{a_{1}}\right) V_{1}^{B \rightarrow a_{1}}\left(m_{a_{1}}^{2}\right)\left(\epsilon_{a_{1}} \cdot \epsilon_{a_{1}}\right)+\frac{i V_{2}^{B \rightarrow a_{1}}\left(m_{a_{1}}^{2}\right)}{\left(m_{B}+m_{a_{1}}\right)}\left(\epsilon_{a_{1}} \cdot p_{B}\right)\left(\epsilon_{a_{1}} \cdot p_{B}\right)\right)
\end{gathered}
$$




$$
\begin{aligned}
& \mathcal{M}\left(\bar{B}^{0} \rightarrow a_{1}^{0} f_{1}\right)=m_{f_{1}} f_{f_{1}}\left\{V_{u b} V_{u d}^{*} a_{2}-V_{t b} V_{t d}^{*}\left[2 a_{3}+a_{4}-2 a_{5}-\frac{1}{2}\left(a_{7}-a_{9}+a_{10}\right)\right]\right\}\left(\frac{2 A^{B \rightarrow a_{1}}\left(m_{f_{1}}^{2}\right)}{\left(m_{B}+m_{a_{1}}\right)} \epsilon_{\mu \nu \alpha \beta} \epsilon_{f_{1}}^{\mu} \epsilon_{a_{1}}^{\nu} p_{B}^{\alpha} p_{a_{1}}^{\beta}\right. \\
& \left.-i\left(m_{B}+m_{a_{1}}\right) V_{1}^{B \rightarrow a_{1}}\left(m_{f_{1}}^{2}\right)\left(\epsilon_{a_{1}} \cdot \epsilon_{f_{1}}\right)+\frac{i V_{2}^{B \rightarrow a_{1}}\left(m_{f_{1}}^{2}\right)}{\left(m_{B}+m_{a_{1}}\right)}\left(\epsilon_{a_{1}} \cdot p_{B}\right)\left(\epsilon_{f_{1}} \cdot p_{B}\right)\right) \\
& +m_{a_{1}} f_{a_{1}}\left\{V_{u b} V_{u d}^{*} a_{2}-V_{t b} V_{t d}^{*}\left[-a_{4}-\frac{3}{2}\left(a_{7}-a_{9}\right)+\frac{1}{2} a_{10}\right]\right\}\left(\frac{2 A^{B \rightarrow f_{1}}\left(m_{a_{1}}^{2}\right)}{\left(m_{B}+m_{f_{1}}\right)} \epsilon_{\mu \nu \alpha \beta} \epsilon_{a_{1}}^{\mu} \epsilon_{f_{1}}^{\nu} p_{B}^{\alpha} p_{f_{1}}^{\beta}\right. \\
& \left.-i\left(m_{B}+m_{f_{1}}\right) V_{1}^{B \rightarrow f_{1}}\left(m_{a_{1}}^{2}\right)\left(\epsilon_{f_{1}} \cdot \epsilon_{a_{1}}\right)+\frac{i V_{2}^{B \rightarrow f_{1}}\left(m_{a_{1}}^{2}\right)}{\left(m_{B}+m_{f_{1}}\right)}\left(\epsilon_{f_{1}} \cdot p_{B}\right)\left(\epsilon_{a_{1}} \cdot p_{B}\right)\right) \\
& \mathcal{M}\left(B^{-} \rightarrow a_{1}^{-} f_{1}\right)=m_{f_{1}} f_{f_{1}}\left\{V_{u b} V_{u d}^{*} a_{2}-V_{t b} V_{t d}^{*}\left[2 a_{3}+a_{4}-2 a_{5}-\frac{1}{2}\left(a_{7}-a_{9}+a_{10}\right)\right]\right\}\left(\frac{2 A^{B \rightarrow a_{1}}\left(m_{f_{1}}^{2}\right)}{\left(m_{B}+m_{a_{1}}\right)} \epsilon_{\mu \nu \alpha \beta} \epsilon_{f_{1}}^{\mu} \epsilon_{a_{1}}^{\nu} p_{B}^{\alpha} p_{a_{1}}^{\beta}\right. \\
& \left.-i\left(m_{B}+m_{a_{1}}\right) V_{1}^{B \rightarrow a_{1}}\left(m_{f_{1}}^{2}\right)\left(\epsilon_{a_{1}} \cdot \epsilon_{f_{1}}\right)+\frac{i V_{2}^{B \rightarrow a_{1}}\left(m_{f_{1}}^{2}\right)}{\left(m_{B}+m_{a_{1}}\right)}\left(\epsilon_{a_{1}} \cdot p_{B}\right)\left(\epsilon_{f_{1}} \cdot p_{B}\right)\right) \\
& +m_{a_{1}} f_{a_{1}}\left\{V_{u b} V_{u d}^{*} a_{1}-V_{t b} V_{t d}^{*}\left(a_{4}+a_{10}\right)\right\}\left(\frac{2 A^{B \rightarrow f_{1}}\left(m_{a_{1}}^{2}\right)}{\left(m_{B}+m_{f_{1}}\right)} \epsilon_{\mu \nu \alpha \beta} \epsilon_{a_{1}}^{\mu} \epsilon_{f_{1}}^{\nu} p_{B}^{\alpha} p_{f_{1}}^{\beta}\right. \\
& \left.-i\left(m_{B}+m_{f_{1}}\right) V_{1}^{B \rightarrow f_{1}}\left(m_{a_{1}}^{2}\right)\left(\epsilon_{f_{1}} \cdot \epsilon_{a_{1}}\right)+\frac{i V_{2}^{B \rightarrow f_{1}}\left(m_{a_{1}}^{2}\right)}{\left(m_{B}+m_{f_{1}}\right)}\left(\epsilon_{f_{1}} \cdot p_{B}\right)\left(\epsilon_{a_{1}} \cdot p_{B}\right)\right) \\
& \mathcal{M}\left(\bar{B}^{0} \rightarrow a_{1}^{+} K_{1}^{-}\right)=m_{K_{1}} f_{K_{1}}\left\{V_{u b} V_{u s}^{*} a_{1}-V_{t b} V_{t s}^{*}\left(a_{4}+a_{10}\right)\right\}\left(\frac{2 A^{B \rightarrow a_{1}}\left(m_{K_{1}}^{2}\right)}{\left(m_{B}+m_{a_{1}}\right)} \epsilon_{\mu \nu \alpha \beta} \epsilon_{K_{1}}^{\mu} \epsilon_{a_{1}}^{\nu} p_{B}^{\alpha} p_{a_{1}}^{\beta}\right. \\
& \left.-i\left(m_{B}+m_{a_{1}}\right) V_{1}^{B \rightarrow a_{1}}\left(m_{K_{1}}^{2}\right)\left(\epsilon_{a_{1}} \cdot \epsilon_{K_{1}}\right)+\frac{i V_{2}^{B \rightarrow a_{1}}\left(m_{K_{1}}^{2}\right)}{\left(m_{B}+m_{a_{1}}\right)}\left(\epsilon_{a_{1}} \cdot p_{B}\right)\left(\epsilon_{K_{1}} \cdot p_{B}\right)\right) \\
& \mathcal{M}\left(\bar{B}^{0} \rightarrow a_{1}^{0} \bar{K}_{1}^{0}\right)=m_{a_{1}} f_{a_{1}}\left\{V_{u b} V_{u s}^{*} a_{2}-V_{t b} V_{t s}^{*} \frac{3}{2}\left(-a_{7}+a_{9}\right)\right\}\left(\frac{2 A^{B \rightarrow K_{1}}\left(m_{a_{1}}^{2}\right)}{\left(m_{B}+m_{K_{1}}\right)} \epsilon_{\mu \nu \alpha \beta} \epsilon_{a_{1}}^{\mu} \epsilon_{K_{1}}^{\nu} p_{B}^{\alpha} p_{K_{1}}^{\beta}\right. \\
& \left.-i\left(m_{B}+m_{K_{1}}\right) V_{1}^{B \rightarrow K_{1}}\left(m_{a_{1}}^{2}\right)\left(\epsilon_{K_{1}} \cdot \epsilon_{a_{1}}\right)+\frac{i V_{2}^{B \rightarrow K_{1}}\left(m_{a_{1}}^{2}\right)}{\left(m_{B}+m_{K_{1}}\right)}\left(\epsilon_{K_{1}} \cdot B\right)\left(\epsilon_{a_{1}} \cdot p_{B}\right)\right) \\
& -m_{K_{1}} f_{K_{1}}\left\{V_{t b} V_{t s}^{*}\left(a_{4}-\frac{1}{2} a_{10}\right)\right\}\left(\frac{2 A^{B \rightarrow a_{1}}\left(m_{K_{1}}^{2}\right)}{\left(m_{B}+m_{a_{1}}\right)} \epsilon_{\mu \nu \alpha \beta} \epsilon_{K_{1}}^{\mu} \epsilon_{a_{1}}^{\nu} p_{B}^{\alpha} p_{a_{1}}^{\beta}\right. \\
& \left.-i\left(m_{B}+m_{a_{1}}\right) V_{1}^{B \rightarrow a_{1}}\left(m_{K_{1}}^{2}\right)\left(\epsilon_{a_{1}} \cdot \epsilon_{K_{1}}\right)+\frac{i V_{2}^{B \rightarrow a_{1}}\left(m_{K_{1}}^{2}\right)}{\left(m_{B}+m_{a_{1}}\right)}\left(\epsilon_{a_{1}} \cdot p_{B}\right)\left(\epsilon_{K_{1}} \cdot p_{B}\right)\right) \\
& \mathcal{M}\left(B^{-} \rightarrow a_{1}^{0} K_{1}^{-}\right)=m_{K_{1}} f_{K_{1}}\left\{V_{u b} V_{u s}^{*}-V_{t b} V_{t s}^{*}\left(a_{4}+a_{10}\right)\right\}\left(\frac{2 A^{B \rightarrow a_{1}}\left(m_{K_{1}}^{2}\right)}{\left(m_{B}+m_{a_{1}}\right)} \epsilon_{\mu \nu \alpha \beta} \epsilon_{K_{1}}^{\mu} \epsilon_{a_{1}}^{\nu} p_{B}^{\alpha} p_{a_{1}}^{\beta}\right. \\
& \left.-i\left(m_{B}+m_{a_{1}}\right) V_{1}^{B \rightarrow a_{1}}\left(m_{K_{1}}^{2}\right)\left(\epsilon_{a_{1}} \cdot \epsilon_{K_{1}}\right)+\frac{i V_{2}^{B \rightarrow a_{1}}\left(m_{K_{1}}^{2}\right)}{\left(m_{B}+m_{a_{1}}\right)}\left(\epsilon_{a_{1}} \cdot p_{B}\right)\left(\epsilon_{K_{1}} \cdot p_{B}\right)\right) \\
& +m_{a_{1}} f_{a_{1}}\left\{V_{u b} V_{u s}^{*}+V_{t b} V_{t s}^{*} \frac{3}{2}\left(a_{7}-a_{9}\right)\right\}\left(\frac{2 A^{B \rightarrow K_{1}}\left(m_{a_{1}}^{2}\right)}{\left(m_{B}+m_{K_{1}}\right)} \epsilon_{\mu \nu \alpha \beta} \epsilon_{a_{1}}^{\mu} \epsilon_{K_{1}}^{\nu} p_{B}^{\alpha} p_{K_{1}}^{\beta}\right. \\
& \left.-i\left(m_{B}+m_{K_{1}}\right) V_{1}^{B \rightarrow K_{1}}\left(m_{a_{1}}^{2}\right)\left(\epsilon_{K_{1}} \cdot \epsilon_{a_{1}}\right)+\frac{i V_{2}^{B \rightarrow K_{1}}\left(m_{a_{1}}^{2}\right)}{\left(m_{B}+m_{K_{1}}\right)}\left(\epsilon_{K_{1}} \cdot p_{B}\right)\left(\epsilon_{a_{1}} \cdot p_{B}\right)\right)
\end{aligned}
$$




$$
\begin{aligned}
& \mathcal{M}\left(B^{-} \rightarrow a_{0}^{-} \bar{K}_{1}^{0}\right)=-m_{K_{1}} f_{K_{1}}\left\{V_{t b} V_{t s}^{*}\left(a_{4}-\frac{1}{2} a_{10}\right)\right\}\left(\frac{2 A^{B \rightarrow a_{1}}\left(m_{K_{1}}^{2}\right)}{\left(m_{B}+m_{a_{1}}\right)} \epsilon_{\mu \nu \alpha \beta} \epsilon_{K_{1}}^{\mu} \epsilon_{a_{1}}^{\nu} p_{B}^{\alpha} p_{a_{1}}^{\beta}\right. \\
& \left.-i\left(m_{B}+m_{a_{1}}\right) V_{1}^{B \rightarrow a_{1}}\left(m_{K_{1}}^{2}\right)\left(\epsilon_{a_{1}} \cdot \epsilon_{K_{1}}\right)+\frac{i V_{2}^{B \rightarrow a_{1}}\left(m_{K_{1}}^{2}\right)}{\left(m_{B}+m_{a_{1}}\right)}\left(\epsilon_{a_{1}} \cdot p_{B}\right)\left(\epsilon_{K_{1}} \cdot p_{B}\right)\right) \\
& \mathcal{M}\left(\bar{B}^{0} \rightarrow \bar{K}_{1}^{0} f_{1}\right)=m_{f_{1}} f_{f_{1}}\left\{V_{u b} V_{u s}^{*} a_{2}-V_{t b} V_{t s}^{*}\left[2 a_{3}-2 a_{5}-\frac{1}{2}\left(a_{7}-a_{9}\right)\right]\right\}\left(\frac{2 A^{B \rightarrow K_{1}}\left(m_{f_{1}}^{2}\right)}{\left(m_{B}+m_{K_{1}}\right)} \epsilon_{\mu \nu \alpha \beta} \epsilon_{f_{1}}^{\mu} \epsilon_{K_{1}}^{\nu} p_{B}^{\alpha} p_{K_{1}}^{\beta}\right. \\
& \left.-i\left(m_{B}+m_{K_{1}}\right) V_{1}^{B \rightarrow K_{1}}\left(m_{f_{1}}^{2}\right)\left(\epsilon_{K_{1}} \cdot \epsilon_{f_{1}}\right)+\frac{i V_{2}^{B \rightarrow K_{1}}\left(m_{f_{1}}^{2}\right)}{\left(m_{B}+m_{K_{1}}\right)}\left(\epsilon_{K_{1}} \cdot p_{B}\right)\left(\epsilon_{f_{1}} \cdot p_{B}\right)\right) \\
& -m_{K_{1}} f_{K_{1}}\left\{V_{t b} V_{t s}^{*}\left(a_{4}-\frac{1}{2} a_{10}\right)\right\}\left(\frac{2 A^{B \rightarrow f_{1}}\left(m_{K_{1}}^{2}\right)}{\left(m_{B}+m_{f_{1}}\right)} \epsilon_{\mu \nu \alpha \beta} \epsilon_{K_{1}}^{\mu} \epsilon_{f_{1}}^{\nu} p_{B}^{\alpha} p_{f_{1}}^{\beta}\right. \\
& \left.-i\left(m_{B}+m_{f_{1}}\right) V_{1}^{B \rightarrow f_{1}}\left(m_{K_{1}}^{2}\right)\left(\epsilon_{f_{1}} \cdot \epsilon_{K_{1}}\right)+\frac{i V_{2}^{B \rightarrow f_{1}}\left(m_{K_{1}^{0}}^{2}\right)}{\left(m_{B}+m_{f_{1}}\right)}\left(\epsilon_{f_{1}} \cdot p_{B}\right)\left(\epsilon_{K_{1}} \cdot p_{B}\right)\right) \\
& \mathcal{M}\left(B^{-} \rightarrow K_{1}^{-} f_{1}\right)=m_{K_{1}} f_{K_{1}}\left\{V_{u b} V_{u s}^{*} a_{1}-V_{t b} V_{t s}^{*}\left(a_{4}+a_{10}\right)\right\}\left(\frac{2 A^{B \rightarrow f_{1}}\left(m_{K_{1}}^{2}\right)}{\left(m_{B}+m_{f_{1}}\right)} \epsilon_{\mu \nu \alpha \beta} \epsilon_{K_{1}}^{\mu} \epsilon_{f_{1}}^{\nu} p_{B}^{\alpha} p_{f_{1}}^{\beta}\right. \\
& \left.-i\left(m_{B}+m_{f_{1}}\right) V_{1}^{B \rightarrow f_{1}}\left(m_{K_{1}}^{2}\right)\left(\epsilon_{f_{1}} \cdot \epsilon_{K_{1}}\right)+\frac{i V_{2}^{B \rightarrow f_{1}}\left(m_{K_{1}}^{2}\right)}{\left(m_{B}+m_{f_{1}}\right)}\left(\epsilon_{f_{1}} \cdot p_{B}\right)\left(\epsilon_{K_{1}} \cdot p_{B}\right)\right) \\
& +m_{f_{1}} f_{f_{1}}\left\{V_{u b} V_{u s}^{*} a_{2}-V_{t b} V_{t s}^{*}\left[2 a_{3}-2 a_{5}-\frac{1}{2}\left(a_{7}-a_{9}\right)\right]\right\}\left(\frac{2 A^{B \rightarrow K_{1}}\left(m_{f_{1}}^{2}\right)}{\left(m_{B}+m_{K_{1}}\right)} \epsilon_{\mu \nu \alpha \beta} \epsilon_{f_{1}}^{\mu} \epsilon_{K_{1}}^{\nu} p_{B}^{\alpha} p_{K_{1}}^{\beta}\right. \\
& \left.-i\left(m_{B}+m_{K_{1}}\right) V_{1}^{B \rightarrow K_{1}}\left(m_{f_{1}}^{2}\right)\left(\epsilon_{K_{1}} \cdot \epsilon_{f_{1}}\right)+\frac{i V_{2}^{B \rightarrow K_{1}}\left(m_{f_{1}}^{2}\right)}{\left(m_{B}+m_{K_{1}}\right)}\left(\epsilon_{K_{1}} \cdot p_{B}\right)\left(\epsilon_{f_{1}} \cdot p_{B}\right)\right)
\end{aligned}
$$

[1] B. Aubert et al. (BABAR Collaboration), Phys. Rev. Lett. 97, 051802 (2006), arXiv:hep-ex/0603050 K. Abe et al. (Belle Collaboration), arXiv:hep-ex/0507096; H. Yang et al. (Belle Collaboration), Phys. Rev. Lett. 94, 111802 (2005), arXiv:hep-ex/0412039 K. Abe et al. (Belle Collaboration), arXiv:hep-ex/0408138; B. Aubert et al. (BaBar Collaboration), Phys. Rev. Lett. 90, 242001 (2003); D. Besson et al. (CLEO Collaboration), Phys. Rev. D 68, 032002 (2003).

[2] G. Nardulli and T. N. Pham, Phys. Lett. B 623, 65 (2005).

[3] A. Ali, G. Kramer and C-D. Lu, Phys. Rev. D 58, 094009 (1998).

[4] Y-H. Chen, H-Y. Cheng, B. Tseng and K-C. Yang, Phys. Rev. D 60, 094014 (1999).

[5] Particle Data Group, W. M. Yao et al., J. Phys. G 33, 1 (2006).

[6] A. C. Katoch and R. C. Verma, J. Phys. G: Nucl. Part . Phys. 22, 1765 (1996).

[7] N. Isgur, D. Scora, B. Grinstein and M. B. Wise, Phys. Rev. D 39, 799(1989).

[8] V. Laporta, G. Nardulli and T. N. Pham, Phys. Rev. D 74, 054035 (2006).

[9] C. H. Chen, C. Q. Geng, Y. K. Hsiao and Z-T. Wei, Phys. Rev. D 72, 054011 (2005).

[10] H. Y. Cheng, Phys. Rev. D 68, 094005 (2003).

[11] D. Scora and N. Isgur, Phys. Rev. D 52, 2783 (1995).

[12] D. Melikhov and B. Stech, Phys. Rev. D 62, 014006 (2000).

[13] H-Y. Cheng and C-K. Chua, Phys. Rev. D 74, 034020 (2006).

[14] J. P. Lee, Phys. Rev. D 74, 074001 (2006).

[15] See for example: H-Y. Cheng, Phys. Rev. D 67, 094007 (2003); M. Suzuki, Phys. Rev. D 47, 1252 (1993); N. Isgur and M. B. Wise, Phys. Lett. B 232, 113 (1989).

[16] D-M. Li and Z. Li, Eur. Phys. J. A 28, 369 (2006).

[17] M. Gronau, D. Pirjol and D. Wyler, Phys. Rev. Lett. 90, 051801 (2003).

[18] M. Gronau and J. Zupan, Phys. Rev. D 73, 057502 (2006).

[19] M. Wirbel, B. Stech and M. Bauer, Z. Phys. C 29, 637 (1985); M. Bauer and M. Wirbel, Z. Phys. C 42, 671 (1989). 
[20] P. Ball and R. Zwicky, Phys. Rev. D 71, 014015 (2005); 71, 014029 (2005).

[21] G. Buchalla, A. J. Buras, and M. E. Lautenbacher, Rev. Mod. Phys. 68, 1125 (1996).

[22] A. J. Buras, Nucl. Phys. B 434, 606 (1995).

[23] A. J. Buras, arXiv:hep-ph/9806471.

[24] A. J. Buras and L. Silvestrini, Nucl. Phys. B 548, 293 (1999).

[25] L. Wolfenstein, Phys. Rev. Lett. 51, 1945 (1983).

[26] A. J. Buras, M. E. Lautenbacher and G. Ostermaier, Phys. Rev. D 50, 3433 (1994).

[27] A. Hocker at al., Eur. Phys. J. C 21, 225 (2001); J. Charles et al., Eur. Phys. J. C 41, 1 (2005).

[28] M. Bona, et al., JHEP 507, 28 (2005); JHEP 603, 080 (2006).

[29] H. Fusaoka and Y. Koide, Phys. Rev. D 57, 3986 (1998).

[30] H. Leutwyler, Nucl. Phys. B (Proc. Suppl.) 64, 223 (1998).

[31] T. Feldmann, P. Kroll and B. Stech, Phys. Rev. D 58, 114006 (1998); Phys. Lett. B 449, 339 (1999).

[32] H. Y. Cheng and B. Tseng, Phys. Rev. D 58, 094005 (1998).

[33] J. L. Diaz-Cruz, G. Lopez Castro and J. H. Munoz, Phys. Rev. D 54, 2388 (1996). 